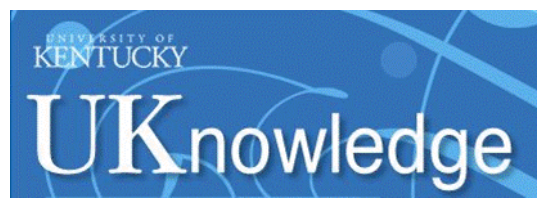

University of Kentucky

UKnowledge

\title{
A PREDICTIVE PROBABILITY INTERIM DESIGN FOR PHASE II CLINICAL TRIALS WITH CONTINUOUS ENDPOINTS
}

\author{
Meng Liu \\ University of Kentucky, meng.liu@uky.edu \\ Digital Object Identifier: https://doi.org/10.13023/ETD.2017.334
}

Right click to open a feedback form in a new tab to let us know how this document benefits you.

\begin{abstract}
Recommended Citation
Liu, Meng, "A PREDICTIVE PROBABILITY INTERIM DESIGN FOR PHASE II CLINICAL TRIALS WITH CONTINUOUS ENDPOINTS" (2017). Theses and Dissertations--Epidemiology and Biostatistics. 15. https://uknowledge.uky.edu/epb_etds/15
\end{abstract}

This Doctoral Dissertation is brought to you for free and open access by the College of Public Health at UKnowledge. It has been accepted for inclusion in Theses and Dissertations--Epidemiology and Biostatistics by an authorized administrator of UKnowledge. For more information, please contact UKnowledge@lsv.uky.edu. 


\section{STUDENT AGREEMENT:}

I represent that my thesis or dissertation and abstract are my original work. Proper attribution has been given to all outside sources. I understand that I am solely responsible for obtaining any needed copyright permissions. I have obtained needed written permission statement(s) from the owner(s) of each third-party copyrighted matter to be included in my work, allowing electronic distribution (if such use is not permitted by the fair use doctrine) which will be submitted to UKnowledge as Additional File.

I hereby grant to The University of Kentucky and its agents the irrevocable, non-exclusive, and royalty-free license to archive and make accessible my work in whole or in part in all forms of media, now or hereafter known. I agree that the document mentioned above may be made available immediately for worldwide access unless an embargo applies.

I retain all other ownership rights to the copyright of my work. I also retain the right to use in future works (such as articles or books) all or part of my work. I understand that I am free to register the copyright to my work.

\section{REVIEW, APPROVAL AND ACCEPTANCE}

The document mentioned above has been reviewed and accepted by the student's advisor, on behalf of the advisory committee, and by the Director of Graduate Studies (DGS), on behalf of the program; we verify that this is the final, approved version of the student's thesis including all changes required by the advisory committee. The undersigned agree to abide by the statements above.

Meng Liu, Student

Dr. Emily Van Meter Dressler, Major Professor

Dr. Steve Browning, Director of Graduate Studies 


\section{A PREDICTIVE PROBABILITY INTERIM DESIGN FOR PHASE II CLINICAL TRIALS WITH CONTINUOUS ENDPOINTS}

DISSERTATION

A dissertation submitted in partial fulfillment of the requirements for the degree of Doctor of Philosophy in the

College of Public Health at the University of Kentucky

$$
\begin{gathered}
\text { By } \\
\text { Meng Liu } \\
\text { Lexington, Kentucky }
\end{gathered}
$$

Co-Directors: Dr. Emily V. Dressler , Professor of Biostatistics

and Dr. Heather Bush , Professor of Biostatistics

Lexington, Kentucky

Copyright (C) Meng Liu 2017 


\section{ABSTRACT OF DISSERTATION}

\section{A PREDICTIVE PROBABILITY INTERIM DESIGN FOR PHASE II CLINICAL TRIALS WITH CONTINUOUS ENDPOINTS}

Phase II clinical trials aim to potentially screen out ineffective and identify effective therapies to move forward to randomized phase III trials. Single-arm studies remain the most utilized design in phase II oncology trials, especially in scenarios where a randomized design is simply not practical. Due to concerns regarding excessive toxicity or ineffective new treatment strategies, interim analyses are typically incorporated in the trial, and the choice of statistical methods mainly depends on the type of primary endpoints. For oncology trials, the most common primary objectives in phase II trials include tumor response rate (binary endpoint) and progression disease-free survival (time-to-event endpoint). Interim strategies are well-developed for both endpoints in single-arm phase II trials.

The advent of molecular targeted therapies, often with lower toxicity profiles from traditional cytotoxic treatments, has shifted the drug development paradigm into establishing evidence of biological activity, target modulation and pharmacodynamics effects of these therapies in early phase trials. As such, these trials need to address simultaneous evaluation of safety as well as proof-of-concept of biological marker activity or changes in continuous tumor size instead of binary response rates.

In this dissertation, we extend a predictive probability design for binary outcomes in the single-arm clinical trial setting and develop two interim designs for continuous endpoints, such as continuous tumor shrinkage or change in a biomarker over time. The two-stage design mainly focuses on the futility stopping strategies, while it also has the capacity of early stopping for efficacy. Both optimal and minimax designs are presented for this two-stage design. The multi-stage design has the flexibility of stopping the trial early either due to futility or efficacy. Due to the intense computation and searching strategy we adopt, only the minimax design is presented for this multi-stage design. The multi-stage design allows for up to 40 interim looks with continuous monitoring possible for large and moderate effect sizes, requiring an overall sample size less than 40 . The stopping boundaries for both designs are based on predictive probability with normal likelihood and its conjugated prior distributions, while the design itself satisfies the prespecified type I and type II error rate constraints. From simulation results, when compared with binary endpoints, both designs well preserve statistical properties across different effect sizes with reduced sample size. We also develop an R package, PPSC, and detail it in chapter four, so that both designs can be freely accessible for use in future phase II clinical trials with the collaborative efforts of biostatisticians. Clinical investigators and 
biostatisticians have the flexibility to specify the parameters from the hypothesis testing framework, searching ranges of the boundaries for predictive probabilities, the number of interim looks involved and if the continuous monitoring is preferred and so on.

KEYWORDS: Predictive Probability, Continuous Endpoints, Single-Arm, Phase II Trials, Interim Strategy, R package 


\section{A PREDICTIVE PROBABILITY INTERIM DESIGN FOR PHASE II CLINICAL TRIALS WITH CONTINUOUS ENDPOINTS}

\section{By}

Meng Liu

Emily Van Meter Dressler

Co-Director of Dissertation

Heather Bush

Co-Director of Dissertation

Steve Browning

Director of Graduate Studies

July 17,2017

Date 
To my parents Hua Li and Hongwei Liu 


\section{ACKNOWLEDGEMENT}

I would like to thank my committee for continued guidance over the past five years. The most important acknowledgement of gratitude I wish to express is to my mentor and supervisor, Dr. Emily Dressler, who is always here when I need help or get into panic. I am grateful for her excellent guidance, caring, patience and providing me with her expertise to tackle each problem in this dissertation work. It was only due to her valuable guidance and cheerful enthusiasm that I was able to complete my dissertation work. I would also like to thank Dr. Heather Bush, who keeps giving me guidance and inspirations since I entered this program, and providing me with all the potential research areas when I had no idea about the future. I would like to thank Dr. Heidi Weiss and Dr. Erin Abner for being in my committee and providing valuable advice for my dissertation to keep me on track. I would like to thank Dr. Aju Mathew for being willing to serve in my committee and providing clinical insights of this work. I would also like to thank Dr. Markos Leggas for being my outside examiner and providing support during the defense process.

I would like to thank everyone in the BBSRF, from whom I have learnt so much about clinical trials as a GRA for the past four years and decided to pursue a career in this field. I would like to thank Dr. Brent Shelton for bringing me to this wonderful and friendly group. I would like to thank Stacey Slone and Jinpeng Liu for helping me out whenever I am stuck in any coding problems. Also, I would like to thank all my cohort classmates, without whom I would never have the courage and confidence to pass through all the courses and exams. It has been a grateful and amazing journey during the past five years.

Finally, I would like to thank my parents for your love, encouragement and constantly support during the past eight years. Thank you both for believing in me, wanting the best for me and inspiring me to follow my dreams. Your love and support is the greatest gift in my life. 


\section{TABLE OF CONTENTS}

Acknowledgments

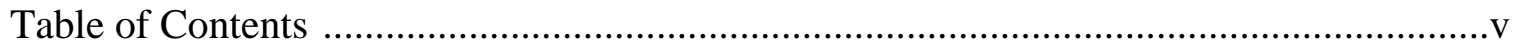

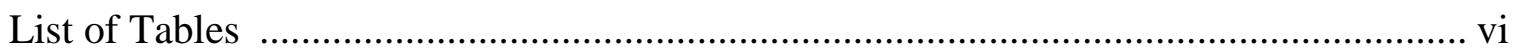

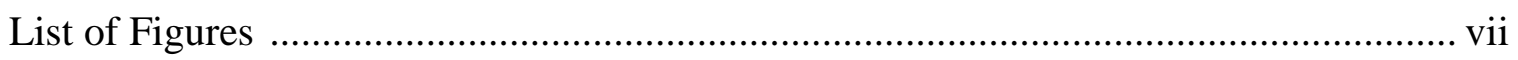

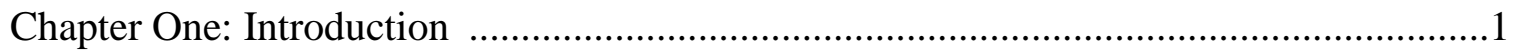

1.1 Current Endpoints in Oncology Phase II Clinical Trials ....................................

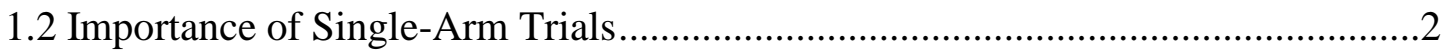

1.3 Current Phase II Trial Designs........................................................................ 3

1.3.1 Importance of Interim Strategies in the Trial ...............................................

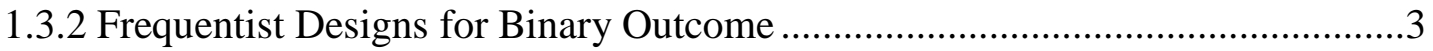

1.3.3 Bayesian Designs for Binary Outcome ........................................................6

1.3.4 Bayesian vs. Frequentist Approaches ............................................................ 9

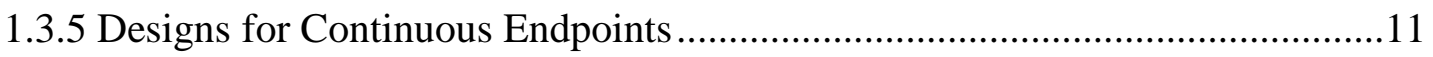

Chapter Two: A Two-Stage Predictive Probability Interim Design for Phase II Clinical Trials with Continuous Endpoints

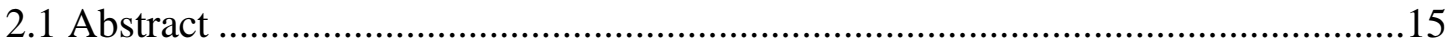

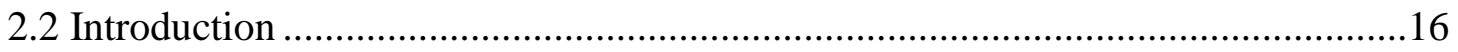

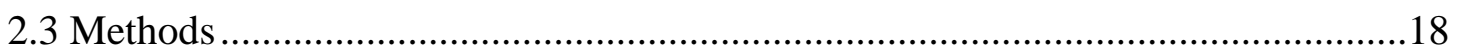

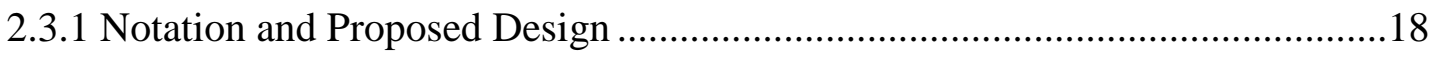

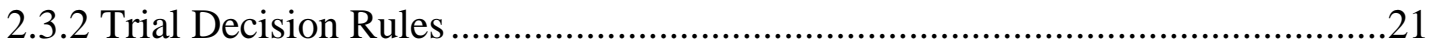

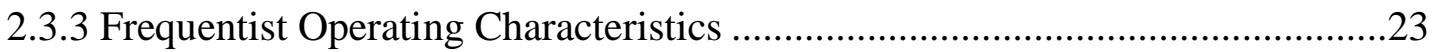

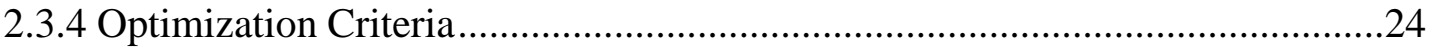

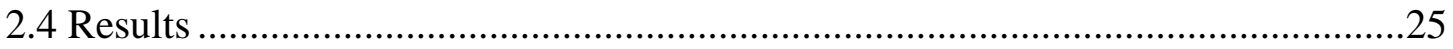

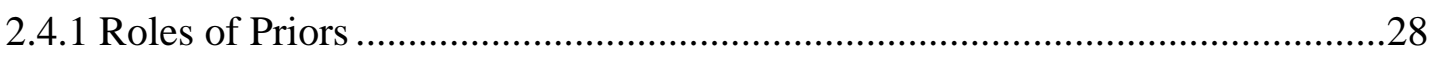

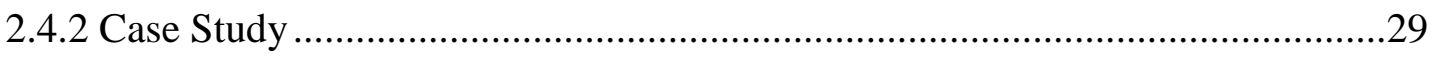

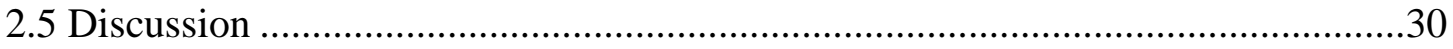

Chapter Three: A Multi-Stage Predictive Probability Interim Design for Phase II Clinical Trials with Continuous Endpoints

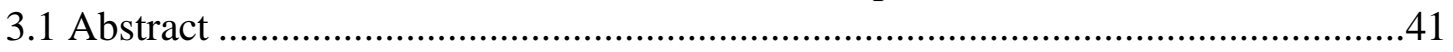

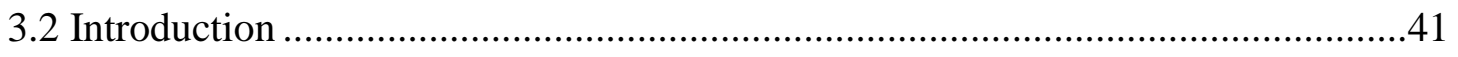

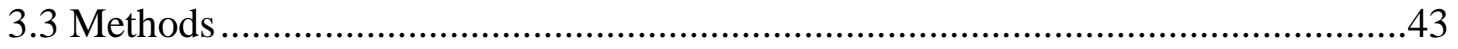

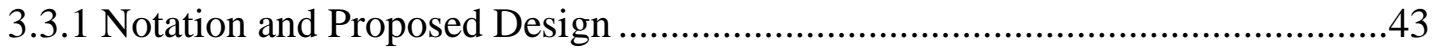

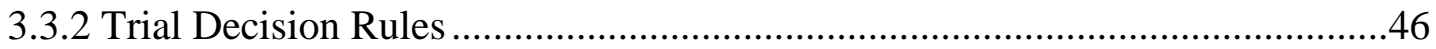


3.3.3 Frequentist Operating Characteristics ..........................................................46

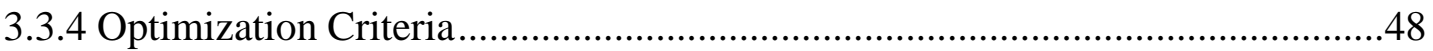

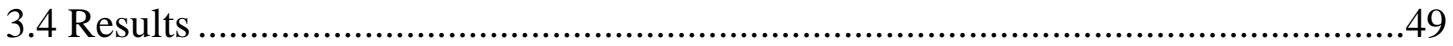

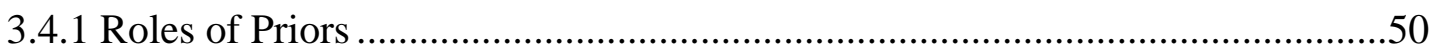

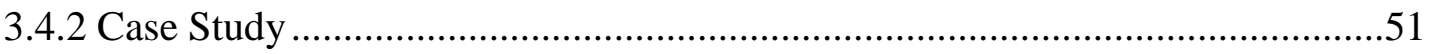

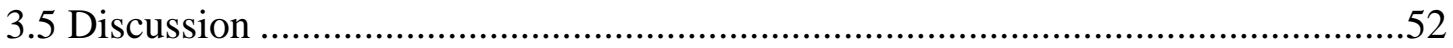

Chapter Four: PPSC: An R Package for Single-Arm Phase II Predictive Probability Design with Continuous Endpoints

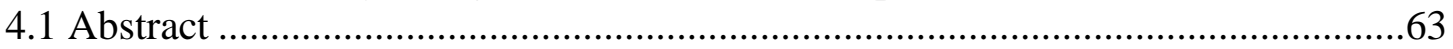

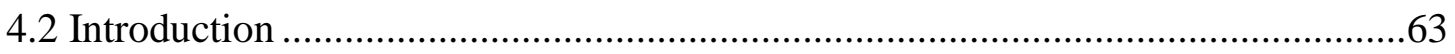

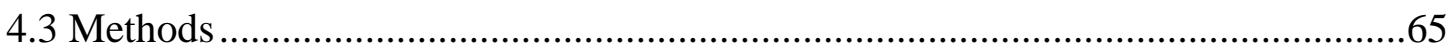

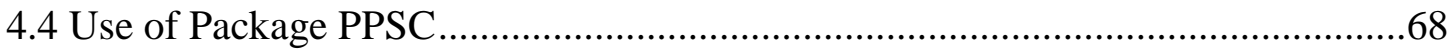

4.4.1 Two-Stage Design for Early Stopping due to Futility: Use of par2stage ….....70

4.4.2 Multi-Stage Design for Early Stopping due to Both Futility and Superiority:

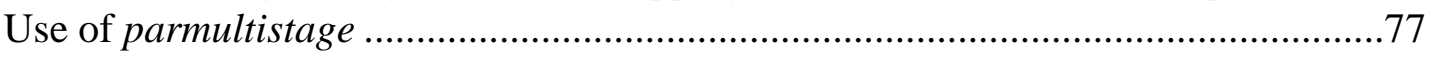

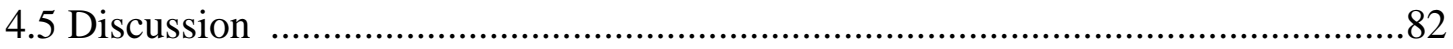

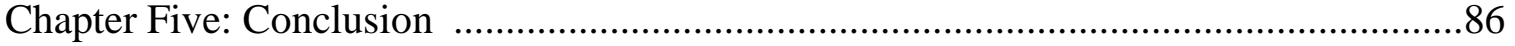

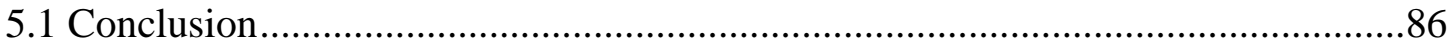

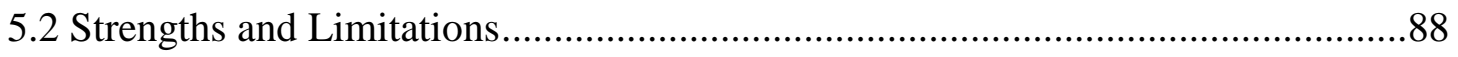

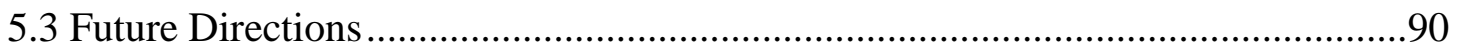

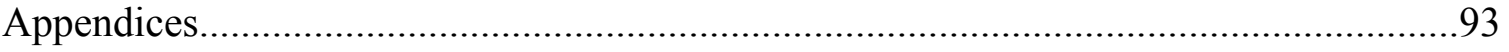

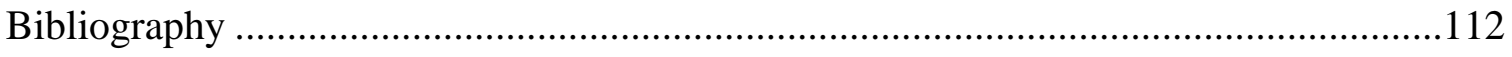

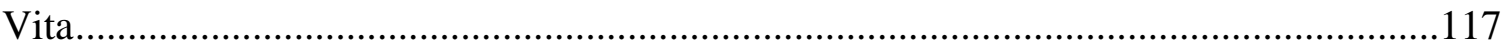




\section{LIST OF TABLES}

Table 2.1 Operating characteristics of optimal design for continuous endpoints with early termination for futility

Table 2.2 Operating characteristics of minimax design for continuous endpoints with early termination for futility .36

Table 2.3 Operating characteristics of optimal design for continuous endpoints with early termination for efficacy.....................................................

Table 2.4 Operating characteristics of minimax design for continuous endpoints with early termination for efficacy............................................... 38

Table 2.5 Operating characteristics of optimal designs with different priors...............39

Table 2.6 Operating characteristics of minimax designs with different priors..............39

Table 2.7 Operating characteristics of optimal design and minimax design for case study.......................................................... 40

Table 3.1 Operating characteristics of minimax multi-stage design for continuous

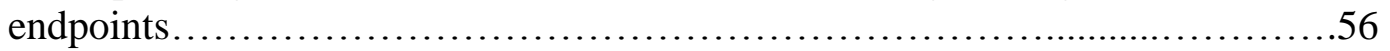

Table 3.2 Operating characteristics of minimax designs with different priors............58

Table 3.3 Operating characteristics of 5-stage and 10-stage minimax design with equal

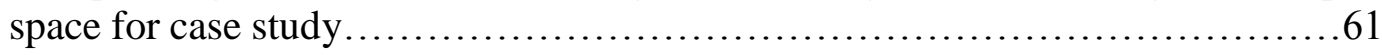

Table 3.4 Operating characteristics of 5-stage unequal space for case study............61

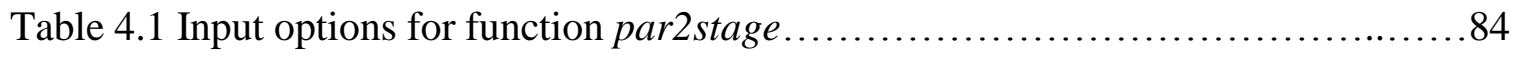

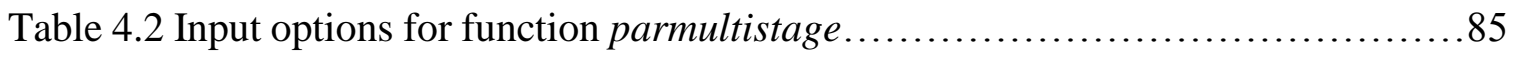




\section{LIST OF FIGURES}

Figure 1.1 Flowchart of Simon's two-stage design............................... 14

Figure 2.1 Proposed two-stage PP design for continuous endpoints.....................34

Figure 3.1: Stopping boundaries for the 10-stage design with 4 different priors ..........60

Figure 3.2: Stopping boundaries for the 5-stage design and 10-stage design for case

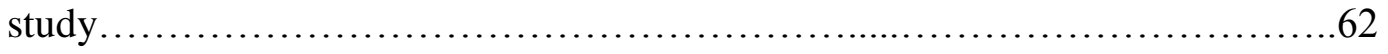




\section{CHAPTER ONE}

\section{Introduction}

\subsection{Current Endpoints in Oncology Phase II Clinical Trials}

In oncology clinical trials, overall survival (OS) has been the gold standard, and considered to be the most clinically relevant and reliable primary endpoint for definitive phase III randomized clinical trials. Therefore, a good endpoint that can predict the overall survival effectively in phase II trials is vital in drug development. In practice, tumor shrinkage is divided into the response rate (RR) according to the Response Evaluation Criteria in Solid Tumors (RECIST) criteria, with a cut-off value of 30\%. Both response rate and progression-free survival (PFS) are commonly used as surrogate endpoints for efficacy in current phase II trials [1, 2].

One of the major challenges in drug development is the efficient design of phase II trials to identify active compounds for phase III testing. The failure to accurately predict effective drugs/treatments in early phase trials causes a disproportionately high incidence of phase III trials with negative results. It has been reported that only $5 \%$ of treatments tested in the phase III setting are approved in oncology [2]. Therefore, there is a need to screen agents in the phase II setting quickly and effectively, while using more robust endpoints predictive of overall survival (OS) and clinical benefit that will predict phase III success, and balance resources to prioritize those therapies deemed promising, especially for novel therapeutics or targeted agents. Endpoints to consider include: continuous tumor size, quality of life outcomes, molecular biomarkers, and imaging assessment [3, 4].

Since trial design, sample size consideration, analysis plans and interim properties completely depend on the type of outcome variable selected, choice of endpoints is of great 
importance before the trial starts. In terms of oncology trials, although response rate has been widely used in practice, studies have shown that designs using binary response rate require many more patients than those using continuous tumor size shrinkage, and lead to loss of statistical efficiency [5, 6]. In addition, drugs can be still active even if they do not lead to a high-level of tumor regression; thus, in this case, a change in a biomarker over time might be more helpful. Further, previous studies have shown that for specific types of cancer, such as patients with metastatic renal cell carcinoma and treated with target therapies, early primary tumor size reduction, which is about $10 \%$ tumor shrinkage, is an independent predictor of improved overall survival. In these situations, RECIST criteria may not be appropriate [1]. Therefore, using continuous tumor size as the primary endpoint has been investigated and shown to be potentially clinically beneficial $[7,8]$.

\subsection{Importance of Single-Arm Trials}

Although randomized phase II trials, including but not limited to selection designs, screening designs, and randomized discontinuation designs, are gaining popularity with clinical researchers, single-arm studies still remain the most utilized design in phase II oncology trials, especially in scenarios where a randomized design is simply not practical [9-11]. Those scenarios include a low likelihood of response to standard therapies, the mechanism of action expected to be cytotoxic, the desired effect size being large, and historical controls remaining stable over time. In particular, with the development of molecular target therapies in oncology recent years, single-arm trials still remain the most feasible design to conduct in practice, especially for smaller centers or investigator initiated trials when the science is novel, and it would be otherwise impossible with available 
resources to obtain preliminary data to then justify a larger, multi-center trial. These situations are quite common for rare tumors or subtypes and gynecologic cancers $[12,13]$.

\subsection{Current Phase II Trial Designs}

\subsubsection{Importance of Interim Strategies in the Trial}

Interim strategies are of great importance in each stage of oncology clinical trials. Developed to protect participants from unsafe drugs or to hasten the general implementation of a beneficial therapy, interim strategies can be utilized to stop trials early either for safety, futility, or efficacy [14]. In addition, interim monitoring in the trial can help identify the need to collect additional data in order to clarify questions of clinical benefit or safety issues that may arise during the trial. It can further help reveal logical problems or issues involving dataquality that need tobe promptly addressed. Thus, incorporating appropriate interim strategies in the trials will facilitate the success of the trial and benefit patients, as well as save time and resources [15-17]. In particular, for phase II trials, which usually have a smaller sample size and relatively short in duration, enough information must be obtained to decide whether the trials should be stopped for lack of evidence suggesting safety or efficacy, or whether the trials should be continued and moved forward to confirmatory phase III testing for evidence demonstrating efficacy. An appropriate stopping rule can lead quickly to an indication and facilitate the decision making.

\subsubsection{Frequentist Designs for Binary Outcome}

During past decades, many statistical methods have been proposed and developed for phase II trial designs. Gehan proposed a two-stage design, in which early termination 
depends on the number of responses in the first stage. If there is no response at the end of the first stage, the trial is stopped for futility; otherwise, continue to the second stage [18]. Simon developed both optimal and minimax design for a single-arm two-stage setting [19], which has been the most widely used single-arm design for phase II studies with binary outcomes. Simon's two-stage design has been extended and developed into different directions in the literature and practice. In particular, Chen extended Simon's design into a three-stage design that allows early termination of the trial due to futility and 10\% average sample size reduction when compared to the two-stage design [20]. Bryant and Day incorporated toxicity into the design to monitor both efficacy and toxicity simultaneously [21], which was then extended into both two-stage and multi-stage designs for dependent variables and control of the type I/II error rates at the same time [22, 23]. Lin et al. extended Simon's design into a two-stage setting that can utilize both primary and secondary endpoints, both of which are assumed to be binary outcomes [24]. Jung et al. developed a graphical method to search for a compromise between optimal and minimax designs [25]. Since two-stage designs are still the most widely used in practice for early phase II trials, especially with the popularity of Simon's two-stage design, we detail the Simon's twostage design below.

\section{Simon's two-stage design}

Simon's two-stage design for terminating the trial early due to futility has been the most commonly applied design in single-arm cancer clinical trials [19]. The design can be illustrated in Figure 1.1. In his design, the primary endpoint is the binary response rate. During the first stage, $n_{1}$ patients are enrolled. If the number of patients with responses within these $n_{1}$ patients is less than or equal to $r_{1}$, the trial should be terminated early for 
futility. Otherwise, we should move forward to the second stage and enroll additional $n_{2}$ patients. If the number of responses at the second stage is less than $r_{2}$, we should stop the trial and claim that this new therapy is not effective and not worth further investigating. $r_{1}$ and $r_{2}$ are the critical values for the number of responses at the end of each stage .

Mathematically, let the true probability of response be $p$, which follows a binomial distribution. Let $p \leq p_{0}$ represents the drug/therapy with low activity that should be screened out from further testing, and $p \geq p_{1}$ represents a drug/therapy with sufficient activity to warrant further phase III testing. $p_{0}$ is some uninteresting level of response rate and $p_{1}$ is a desirable target level. A typical hypothesis testing can be stated:

$$
H_{0}: p \leq p_{0} \text { vs. } H_{1}: p \geq p_{1}
$$

Let $\mathrm{X}$ denote the number of responses at the $1^{\text {st }}$ stage.

1. If the drug is rejected at the end of the $1^{\text {st }}$ stage, the total number of responses at the end of the $1^{\text {st }}$ stage is less than $r_{1}$. Thus, the probability of rejecting a drug (failing to reject the null hypothesis) with response probability $p$ is:

$$
\operatorname{Pr}\left(X \leq r_{1}\right)=\sum_{x=0}^{r_{1}} b\left(x ; p, n_{1}\right)=B\left(r_{1} ; p, n_{1}\right)
$$

where $b\left(x ; p, n_{1}\right)$ is the probability mass function (pmf) of the binomial distribution with parameters $p$ and $n_{1} . B\left(r_{1} ; p, n_{1}\right)$ is the cumulative distribution function $(c d f)$ of binomial distribution representing $\operatorname{Pr}\left(X \leq r_{1}\right)$ with parameters $p$ and $n_{1}$.

2. If the drug is rejected at the end of the $2^{\text {nd }}$ stage, then the total number of responses in the $2^{\text {nd }}$ stage is less than $r_{2}$, and the probability of rejecting the drug can be expressed as: 


$$
\sum_{x=r_{1}+1}^{\min \left(r, n_{1}\right)} b\left(x ; p, n_{1}\right) B\left(r-x ; p, n_{2}\right)
$$

Therefore, the probability of rejecting the drug, $\operatorname{Pr}\left(\right.$ reject the drug $\mid p, n_{1}, n_{2}, r_{1}$, ), with response probability $p$ can be expressed as:

$$
B\left(r_{1} ; p, n_{1}\right)+\sum_{x=r_{1}+1}^{\min \left(r, n_{1}\right)} b\left(x ; p, n_{1}\right) B\left(r-x ; p, n_{2}\right)
$$

The first part of the formula above is the probability of failing to reject the null hypothesis at the end of the $1^{\text {st }}$ stage, and the second part of the formula above is the probability of failing to reject the null hypothesis at the end of $2^{\text {nd }}$ stage.

Thus, the type I and type II error can be calculated as follows:

$$
\begin{gathered}
\alpha=1-\operatorname{Pr}\left(\text { reject the } \operatorname{drug} \mid p_{0}, n_{1}, n_{2}, r_{1}, r_{2}\right) \\
\beta=\operatorname{Pr}\left(\text { reject the drug } \mid p_{1}, n_{1}, n_{2}, r_{1}, r_{2}\right)
\end{gathered}
$$

The probability of early termination $\left[P E T=B\left(r_{1} ; p, n_{1}\right)\right]$ and the expected sample size under the null hypothesis is

$$
E(N)=n_{1}+(1-P E T) n_{2}
$$

In Simon's two-stage design paper, both optimal and minimax designs were introduced [19]. The optimal design is defined to minimize the average sample size under the null hypothesis, while the minimax design is simply to minimize the total sample size required for the two stages. Both designs satisfy the pre-specified nominal type I and type II error rates.

\subsubsection{Bayesian Designs for Binary Outcome}

Bayesian approaches, which make inferences about the unknown parameters of interest based on observed data from realized sample, have gained popularity during the 
past decades. Compared with frequentist approaches, Bayesian approaches can be appealing as they incorporate information before and during a trial to design and monitor progress $[26,27]$.

In terms of phase II clinical trial designs, especially the interim designs, there has been vast development in the Bayesian framework. In particular, Thall and Simon introduced a class of phase II Bayesian clinical trial interim designs [28]. Tan and Machin proposed a two-stage design based on the posterior distribution [29]. Their design was then extended into a two-stage design based on predictive distribution [30-32]. Mayo and Gajewski utilized informative conjugate priors to assess the sample size calculation in the Bayesian setting [33]. Wang et al. developed a Bayesian single-arm two-stage design incorporating both frequentist and Bayesian error rates [34]. Shi and Yin proposed a twostage design with switching hypothesis, in which the first stage is a single-arm study, while the second stage is a two-arm study; the design controls both frequentist and Bayesian error rates [35].

Predictive probabilities (PP) approach for clinical trial designs was originally introduced by Herson for binary endpoints, but did not control the type I and type II error rates throughout the trial [36]. Lee and Liu developed a Bayesian alternative based on PP for binary response rate endpoints [37]. Their PP design allows for continuous monitoring at pre-specified intervals, is efficient in stopping a trial early if the intervention is not efficacious, and controls type I and II error rates. Their design was then extended into a randomized setting by Yin et al. for binary endpoints [38]. Dong et al. incorporated the Bayesian error rates into Lee and Liu's PP design and developed a two-stage single-arm 
design for binary endpoints [39]. Since our design is a straightforward extension to Lee and Liu's PP design, their design is detailed below.

\section{Lee and Liu's Predictive Probability Design}

Oftentimes, when we do not have sufficient data to obtain a definitive conclusion, we need to figure out if the trend continues, the probability of reaching a conclusive result by the end of the study. Lee and Liu developed an efficient and flexible design incorporating the properties of predictive probability under Bayesian framework [37]. Under the same hypothesis testing framework,

$$
H_{0}: p \leq p_{0} \text { vs. } H_{1}: p \geq p_{1}
$$

At any stage, let $X$ denote the number of responses in the current $n$ patients, which follows a binomial distribution with parameters $n$ and $p$. Thus, the likelihood function for the observed data $x$ is

$$
L_{x}(p)=p^{x}(1-p)^{n-p}
$$

We assume the prior distribution of the response rate $\pi(p)$ follows a beta distribution, beta $\left(a_{0}, b_{0}\right)$, which is a conjugate prior for the binomial distribution representing the investigator's previous knowledge or belief of the efficacy of the new agent. The prior mean is $a_{0} /\left(a_{0}+b_{0}\right)$, with $\left(a_{0}+b_{0}\right)$ to be considered as a measure of the amount of information contained in the prior. $a_{0}$ and $b_{0}$ can be considered as the number of responses and the number of non-responses, respectively. Thus, the posterior distribution of the response rate follows a beta distribution beta $\left(a_{0}+x, b_{0}+n-x\right)$.

Therefore, the number of responses in the potential future $m=N_{\text {max }}-n$ patients, $Y$, follows a beta-binomial distribution, where $N_{\max }$ is a maximum accrual of patients, 


$$
\text { beta }-\operatorname{binomial}\left(m ; a_{0}+x ; b_{0}+n-x\right)
$$

The posterior probability of $P$ when $Y=i ; X=x$ can be written as

$$
\operatorname{beta}\left(a_{0}+x+i ; b_{0}+N_{\max }-x-i\right),
$$

giving

$$
\begin{gathered}
\text { Predictive Probability }(P P)=\sum_{i=0}^{m} \operatorname{Prob}(Y=i \mid x) * I\left(\operatorname{Prob}\left(P \geq p_{0} \mid x, Y=i\right) \geq P_{T}\right) \\
=\sum_{i=0}^{m}\left(\operatorname{Prob}(Y=i \mid x) * I\left(B i \geq P_{T}\right)\right)=\sum_{i=0}^{m}\left(\operatorname{Prob}(Y=i \mid x) * I_{i}\right)
\end{gathered}
$$

where $B i=\operatorname{Prob}\left(P \geq p_{0} \mid x, Y=i\right)$, which measures the probability that the response rate is larger than $p_{0}$ given $x$ responses in $n$ patients in the current data, and $i$ patients in the $m$ future patients; and $\operatorname{Prob}(Y=i \mid x)$ is the probability of observing $i$ responses in $m$ patients given current data $x$, where $Y$ follows a beta-binomial distribution.

Choose $\theta_{L}$ as a small positive number and $\theta_{U}$ as a large positive number, between 0 and 1 .

If $P P \leq P_{L}$ then stop the trial and claim the drug is not promising;

If $P P \geq P_{U}$ then stop the trial and reject the null hypothesis;

Otherwise, continue to the next stage until reaching the maximum sample size.

Meanwhile, Lee and Liu's PP design also meets the pre-specified frequentist type I and II error rates. By summing up the probability of rejecting the null hypothesis at each stage, the actual type I and type II error can be calculated.

\subsubsection{Bayesian vs. Frequentist Approaches}

Two statistical philosophies dominate trial designs and analyses. The frequentist approach centers on the idea that data is a repeatable random sample of the true population and underlying parameters are fixed and constant. Studies are designed to meet pre- 
specified type I and type II errors, and decisions are based on point estimates, standard errors, and 95\% confidence intervals [40, 41]. The Bayesian approach observes fixed data from the realized sample, and parameters are unknown and need to be described probabilistically. Bayesian studies start with a prior distribution based on previous studies or historical rates, and make decisions based on accrued data with an updated posterior distribution using means, quantiles, and posterior probabilities [42, 43].

There are many reviews and editorials discussing the controversies surrounding these two approaches; however, there is no argument that both of these strategies are implemented in clinical trials today [44-47]. Frequentist approaches have historically been the more popular strategy with designed a priori stopping rules and number of allowed looks at the data. Some may consider these rules inflexible, but many favor this approach as it allows for a more final yes/no decision at the conclusion of a study [48]. It also does not require sophisticated computer programs or simulations to conduct these tests. Some Bayesian approaches can be appealing as they incorporate information before and during a trial to design and monitor progress respectively $[26,27,37,49,50]$.

There is generally more flexibility and consistency for making inferences; however, these approaches often require more computer programming, can be complex to implement without resources and staff knowledgeable in these methods, and face challenges with scientific and regulatory review panels without sufficient justification of design choice [51, $52]$. 


\subsubsection{Designs for Continuous Endpoints}

\section{Frequentist Designs for Continuous Endpoints}

As mentioned above, using response rate endpoints will lead to loss of statistical efficiency. Therefore, previous studies have proposed using continuous tumor shrinkage as the primary endpoint. It was originally proposed by Lavin [53], which was a single-arm and single stage design. His work was then extended into a two-arm randomized design with no planned interim analysis [54]. Wason et al. proposed a frequentist two-stage design based on continuous tumor shrinkage and claimed that there was a $37 \%$ reduction in sample size [5]. Their group also developed a randomized multi-stage $\delta$-minimax design for continuous outcomes [55]. Hsiao et al. also proposed a frequentist two-stage design based on continuous efficacy endpoints, using a general quasi-Newton optimizer to find the optimal design [56]. Other studies using continuous endpoints, especially in a randomized scenario, have also been proposed in the literature. In particular, Whitehead developed a two-stage randomized design with normally distributed endpoints, and the trial decision rule was based on a normalizing transformed p-value [57]. Their study was then extended into a randomized two-stage design that can minimize the maximum expected sample size across all possible treatment effects [58].

\section{Bayesian Designs for Continuous Endpoints}

In the Bayesian Framework, Brutti et al. proposed a Bayesian approach based on posterior distribution with a class of prior distribution to determine the same size of sequential designs [59]. Their group also proposed a Bayesian two-stage design for continuous endpoints using design priors and analysis priors [32]. The design prior is to help plan the trial under expected scenarios, and the analysis prior is to represent the prior 
knowledge of the parameter being studied. In a randomized two-arm trial setting, Dmitrienko and Wang discussed the stopping rules incorporating Bayesian predictive approach in terms of normally distributed outcome. They also compared purely Bayesian predictive probability approach with frequentist as well as mixed Bayesian-frequentist methods, such as conditional and predictive power stopping rules [60].

On the other hand, group sequential designs play an important role in current multistage clinical trials practice. When compared to single-stage designs, multi-stage designs have the advantage that the information obtained from the interim data can be incorporated into the design, which makes it more efficient and stops the trial early. Fleming proposed a multiple testing procedure for one-sample design to allow for early termination while maintain the statistical properties for binary endpoints[61]. Pocock's design as well as O'Brien and Fleming's design are still the two most commonly used group-sequential options $[62,63]$.

To our knowledge, there has not been any published Bayesian predictive work while preserving frequentist properties, focusing on a single-arm setting for continuous outcomes. As an example of continuous endpoints, tumor size change has been discussed to be a potential biomarker for predicting survival in early phase trials [64]. Sometimes, when tumor does not have a high level of shrinkage, and there is a biomarker associated with the treatment effect, the continuous change in this biomarker will be a more appropriate endpoint. Further, design based on continuous biomarkers offers greater statistical power than a categorical endpoint, since dividing the continuous endpoints into categorical variables leads to loss of statistical efficiency. Therefore, there is a need to 
develop interim strategies to incorporate continuous endpoints to accommodate these novel biomarker endpoints. 
Figure 1.1 Flowchart of Simon's two-stage design

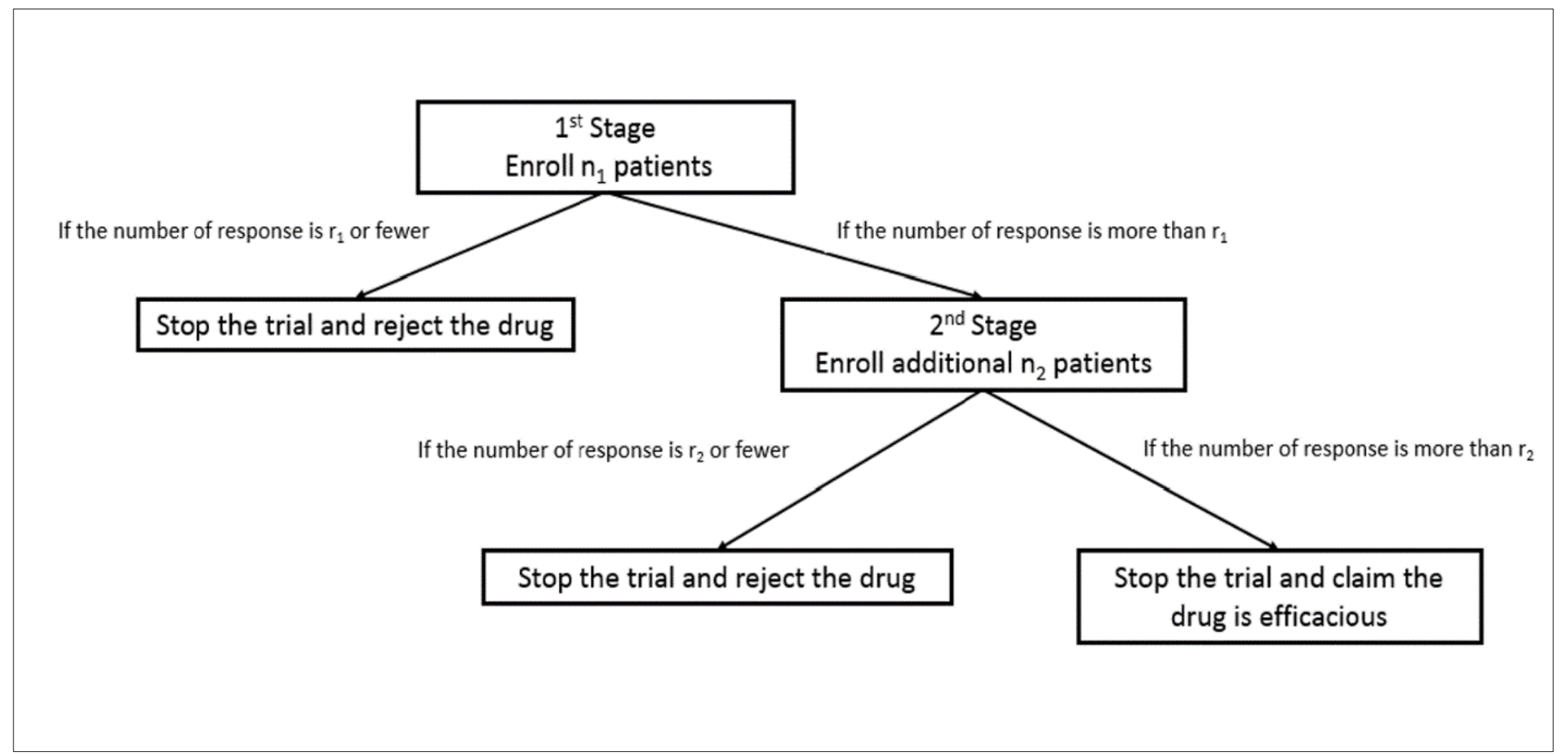




\section{CHAPTER TWO}

\section{A Two-Stage Predictive Probability Interim Design for Phase II Clinical Trials with Continuous Endpoints}

\subsection{Abstract}

Molecular targeted therapies often come with lower toxicity profiles than traditional cytotoxic treatments, thus shifting the drug development paradigm into establishing evidence of biological activity, target modulation and pharmacodynamics effects in early phase trials. Therefore, these trials need to address simultaneous evaluation of safety, proof-of-concept biological marker activity or changes in continuous tumor size instead of binary response rate. Interim analyses are typically incorporated in the trial due to concerns regarding excessive toxicity and ineffective new treatment. There is a lack of interim strategies developed to monitor futility and/or efficacy for these types of continuous outcomes, especially in single-arm phase II trials. We propose a two-stage design based on predictive probability to accommodate continuous endpoints, using continuous tumor shrinkage as an example and assuming a normal distribution with known variance. Although the design is primarily for early termination due to futility, it also has the capacity to stop the trial early for efficacy. Simulation results and the case study demonstrated that the proposed design can incorporate an interim stop for futility while maintaining desirable design properties. As expected, using continuous tumor size change resulted in reduced sample sizes for both optimal and minimax designs. A limited exploration of various priors was performed and shown to be robust. As research rapidly moves to incorporate more molecular targeted therapies, this design will accommodate new 
types of outcomes while allowing for flexible stopping rules to continue optimizing trial resources and prioritizing agents with compelling early phase data.

Keywords: Phase II trials; single-arm; continuous endpoints; predictive probability; interim

\subsection{Introduction}

Phase II clinical trials aim to potentially screen out ineffective, and identify effective therapies to move forward to the randomized phase III setting. Although randomized phase II trials, including but not limited to selection designs, screening designs, and randomized discontinuation designs, are gaining popularity with clinical researchers, single-arm studies still remain the most utilized design in phase II oncology trials, especially in scenarios where a randomized design is simply not practical [9-11]. These can include a low likelihood of response to standard therapies, the mechanism of action is expected to be cytotoxic, the desired effect size is large, and historical controls remain stable over time. Further, given the shifting molecular landscape in oncology, smaller single-arm trials are most feasible to conduct in terms of targeted therapeutic agents, especially for smaller centers or investigator initiated trials when the science is novel. It would be otherwise impossible with available resources to obtain preliminary data to then justify a larger, multi-center trial. The single-arm trials are also feasible for rare tumors or subtypes, such as NSCLC patients with ERBB2 or BRAF alteration and gynecologic cancers $[12,65]$.

Due to concerns regarding excessive toxicity and/or ineffective new treatment strategies, interim analyses are encouraged and typically incorporated. The choice of 
statistical methods depends on the type of primary endpoint. For oncology studies, the most common way of assessing tumor shrinkage is to dichotomize patients by response rate according to RECIST [1]. Accordingly, Simon's two-stage design is the most widely used design for this binary endpoint in single-arm trials [19], which allows for one early stop due to futility. There are many extensions of Simon's design, including composite endpoints or multiple interim looks [20,21]. However, studies have shown that using binary response rate, besides loss of statistical efficiency, more patients are required than those using continuous tumor size shrinkage and drugs can be active even if they do not lead to high-levels of tumor regression $[5,6]$.

Besides tumor size changes, continuous outcomes could also include biomarker changes over time from baseline as preliminary evidence of biologic mechanism. Examples include but are not limited to measuring proliferation by Ki67, apoptosis, genetic alterations, or immunity measures of cytokines or TNF-alpha. Detected in circulation, excretion or biopsies, these biomarkers must be robust and clinically validated, especially if utilized as a primary outcome [66]. Thus, RECIST may not be appropriate in such circumstances and using continuous tumor size as primary outcome has been investigated and shown to be potentially clinical beneficial [8].

The idea of using continuous tumor size changes as primary endpoint, was originally proposed by Lavin [53], with his work extended into a two-arm randomized single-stage design with no interim considerations [54]. Wason et al. extended the frequentist Simon's two-stage design for continuous outcomes under normal distribution assumptions [5]. He compared the optimal and minimax designs for continuous tumor size reduction, claiming that using continuous endpoints resulted in lower expected and 
maximum sample sizes as compared to binary counterparts. Hsiao et al. also proposed a frequentist two-stage design based on continuous efficacy endpoints, using a general quasiNewton optimizer to find the optimal design [56]. Bayesian interim strategies involving binary endpoints have been extensively studied [28, 29]. In terms of normally distributed outcomes, the predictive probability approach for Bayesian interim strategies has been proposed only in a randomized setting [60].

In this paper, we extend Lee and Liu's PP design for continuous endpoints in the single-arm trial setting. For purposes of illustration, we use continuous tumor size change as an example of a continuous clinical endpoint, as it has been discussed as a potential surrogate for survival in early phase trials. However, this design would be applicable for any continuous outcome [64]. In section 2.3, we provide explicit formulae for the continuous PP design optimization criteria. In section 2.4, we report simulation results with a case study and conclude with a discussion in section 2.5 .

\subsection{Methods}

\subsubsection{Notation and Proposed Design}

In a phase II trial with continuous endpoints, the hypothesis testing framework can be described as follows:

$$
H_{0}: \mu \leq \mu_{0} \text { vs. } H_{1}: \mu \geq \mu_{1}
$$

where $\mu_{0}$ represents some maximum uninteresting efficacy threshold or efficacy from the standard treatment, and $\mu_{1}$ represents a target efficacy threshold from a new treatment that would warrant additional study. A trial is usually designed such that 


$$
\begin{aligned}
& P\left(\text { Accept New Treatment } \mid H_{0}\right) \leq \alpha \\
& P\left(\text { Reject New treatment } \mid H_{1}\right) \leq \beta
\end{aligned}
$$

where $\alpha$ and $\beta$ represent type I and type II error rate constraints.

The proposed extension of Lee and Liu's PP design for continuous endpoints is described in Figure 2.1. For notation purposes, we define the percentage decrease in the sum of the longest diameter of the target lesions compared to baseline as "tumor size change" and assume it is normally distributed with mean $\mu$ and variance $\sigma^{2}$, where $\sigma^{2}$ is assumed to be known. Positive values of $\mu$ represent shrinkage in the tumor size. We choose a normal conjugate prior distribution $N\left(\theta, \tau^{2}\right)$ for $\mu$, which represents the investigator's previous knowledge of the tumor size change from standard treatment or historical control. We also define the effective sample size for the prior (ESS) as $\frac{\sigma^{2}}{\tau^{2}}$, according to previous literature [67]. ESS can be understood as the amount of information and weight contained in the prior. We set the maximum accrual of patients to $N_{\max }$. The number of patients in the first stage is $n_{1}$ and in the second stage is $n_{2}$, where $n_{1}+n_{2}=$ $N_{\text {max }}$. After recruiting $n_{1}$ patients in the first stage, the mean percentage change of the tumor size from these $n_{1}$ patients is denoted as $\bar{X}_{n_{1}}$. Thus, the corresponding posterior distribution on $\mu$ is normally distributed with

$$
\begin{gathered}
E\left(\mu \mid x_{1}, x_{2}, \ldots, x_{n_{1}}\right)=\frac{\sigma^{2} \theta}{n_{1} \tau^{2}+\sigma^{2}}+\frac{n_{1} \tau^{2}}{n_{1} \tau^{2}+\sigma^{2}} \bar{X}_{n_{1}} \\
\operatorname{Var}\left(\mu \mid x_{1}, x_{2}, \ldots, x_{n_{1}}\right)=\frac{\tau^{2} \sigma^{2}}{n_{1} \tau^{2}+\sigma^{2}}
\end{gathered}
$$


Further, if we were to observe the future $n_{2}$ patients, the new posterior distribution of the tumor size change, $\mu_{*}$, follows a normal distribution with the mean $\mu_{p}^{*}$ and $\sigma_{p}^{* 2}$, where

$$
\begin{gathered}
\mu_{p}^{*}=\frac{\sigma^{2} \theta}{\left(n_{1}+n_{2}\right) \tau^{2}+\sigma^{2}}+\frac{n_{1} \tau^{2}}{\left(n_{1}+n_{2}\right) \tau^{2}+\sigma^{2}} \bar{X}_{n_{1}}+\frac{n_{2} \tau^{2} \bar{X}_{n_{2}}}{\left(n_{1}+n_{2}\right) \tau^{2}+\sigma^{2}} \\
\sigma_{p}^{* 2}=\frac{\tau^{2} \sigma^{2}}{\left(n_{1}+n_{2}\right) \tau^{2}+\sigma^{2}}
\end{gathered}
$$

Similar to Lee and Liu's PP design, we define $B_{i}=\operatorname{Prob}\left(\mu_{*}>\mu_{0} \mid \bar{X}_{n_{1}}, \bar{X}_{n_{2}}\right) . B_{i}$ measures the probability that the mean tumor size change is larger than $\mu_{0}$, given the mean tumor size change in current $n_{1}$ patients and future $n_{2}$ patients. Comparing $B_{i}$ to a threshold $P_{T}$, we aim to conclude the treatment is efficacious when $B_{i}>P_{T}$,

$$
\begin{gathered}
B_{i}=\operatorname{Prob}\left(\mu_{*}>\mu_{0} \mid \bar{X}_{n_{1}}, \bar{X}_{n_{2}}\right)=1-\operatorname{Prob}\left(\mu_{*} \leq \mu_{0} \mid \bar{X}_{n_{1}}, \bar{X}_{n_{2}}\right) \\
=1-\Phi\left(\frac{\mu_{0}-\mu_{p}^{*}}{\sigma_{p}^{*}}\right)>P_{T}
\end{gathered}
$$

where $\Phi($.$) represents the cumulative density function (CDF) of the standard normal$ distribution. The above inequality (1) can be derived into an inequality of $\bar{X}_{n_{2}}$ regarding $P_{T}$

$$
\bar{X}_{n_{2}}>\frac{\left(\left(n_{1}+n_{2}\right) \tau^{2}+\sigma^{2}\right)\left(\mu_{0}-\sigma_{p}^{*} \Phi^{-1}\left(1-P_{T}\right)\right)-\sigma^{2} \theta-\tau^{2} n_{1} \bar{X}_{n_{1}}}{n_{2} \tau^{2}}=\delta
$$

The posterior predictive distribution of tumor size change from the future $n_{2}$ patients follows a normal distribution $N\left(\mu_{p}, \sigma_{p}^{2}\right)$, where

$$
\mu_{p}=\frac{\sigma^{2} \theta}{n_{1} \tau^{2}+\sigma^{2}}+\frac{n_{1} \tau^{2}}{n_{1} \tau^{2}+\sigma^{2}} \bar{X}_{n_{1}}
$$




$$
\sigma_{p}^{2}=\frac{\sigma^{2}}{n_{2}}+\frac{\tau^{2} \sigma^{2}}{n_{1} \tau^{2}+\sigma^{2}}
$$

Then we define

Predictive probability $(P P)=\operatorname{Prob}\left(\bar{X}_{n_{2}}>\delta \mid \bar{X}_{n_{1}}\right)$

$$
=1-\operatorname{Prob}\left(\left(\bar{X}_{n_{2}} \leq \delta \mid \bar{X}_{n_{1}}\right)=1-\Phi\left(\frac{\delta-\mu_{p}}{\sigma_{p}}\right)\right.
$$

$P P$ is a measurement that can be used to determine whether the trial should be stopped early either due to efficacy or futility, or whether the trial should be continued given current inclusive data. A high PP means that given current data, the treatment is likely to be promising by the end of the study; whereas a low PP suggests that the treatment may not have sufficient activity.

\subsubsection{Trial Decision Rules}

We use similar decision rules as those described in Lee and Liu's PP design. $P_{L}$ and $P_{U}$ are defined as lower and upper bounds for the predictive probability, respectively. Usually, $P_{L}$ is chosen as a small positive number and $P_{U}$ is a large positive number, both of which are between 0 and 1 . If $P P$ is less than a small $P_{L}$, it indicates that it is unlikely that the true mean will be larger than $\mu_{0}$ at the end of the trial given the information from the patients in the first stage. Alternatively, if $P P$ is greater than a large $P_{U}$, it means that it is very possible that we can claim the test drug or treatment is effective at the end of the trial based on current data. The decision rules in terms of $P P$ are: 
- If $P P<P_{L}$, stop the trial early for futility;

- If $P P>P_{U}$, stop the trial early for efficacy;

- Otherwise, continue to enroll patients to the next stage.

By comparing $P P$ with $P_{L}$ and $P_{U}$ and solving for $\bar{X}_{n_{1}}$, we can obtain $\bar{X}_{L}$ and $\bar{X}_{U}$, which are defined as the lower and upper bounds for the sample mean from patients in the first stage, $\bar{X}_{n_{1}}$, respectively.

$$
\begin{gathered}
\bar{X}_{L}=\frac{\frac{k_{1} k_{2} \mu_{0}-k_{1} k_{2} \sigma_{p}^{*} \Phi^{-1}\left(1-P_{T}\right)-n_{2} \tau^{2} k_{2}\left(\sigma_{p} \Phi^{-1}\left(1-P_{L}\right)\right)}{k_{2}+n_{2} \tau^{2}}-\sigma^{2} \theta}{n_{1} \tau^{2}} \\
\bar{X}_{U}=\frac{\frac{k_{1} k_{2} \mu_{0}-k_{1} k_{2} \sigma_{p}^{*} \Phi^{-1}\left(1-P_{T}\right)-n_{2} \tau^{2} k_{2}\left(\sigma_{p} \Phi^{-1}\left(1-P_{U}\right)\right)}{k_{2}+n_{2} \tau^{2}}-\sigma^{2} \theta}{n_{1} \tau^{2}}
\end{gathered}
$$

where $k_{1}=\left(n_{1}+n_{2}\right) \tau^{2}+\sigma^{2}, k_{2}=n_{1} \tau^{2}+\sigma^{2}$.

Thus, in our design, the decision rule can equivalently be described as follows in terms of $\bar{X}_{L}$ and $\bar{X}_{U}$ :

- If $\bar{X}_{n_{1}}<\bar{X}_{L}$, stop the trial at the end of the first stage for futility, reject the alternative hypothesis and claim the treatment is not promising;

- If $\bar{X}_{n_{1}}>\bar{X}_{U}$, stop the trial at the end of the first stage for efficacy, reject the null hypothesis and claim the study therapy is promising;

- Otherwise, if $\bar{X}_{L} \leq \bar{X}_{n_{1}} \leq \bar{X}_{U}$ continues to the second stage with additional $n_{2}$ patients. 


\subsubsection{Frequentist Operating Characteristics}

For the above described futility design, the probability of failing to reject $H_{0}$ with the true mean level $\mu$ is given by

$$
\begin{aligned}
\varphi\left(\mu, \sigma, n_{1}, n_{2}, \bar{X}_{n_{1}}, \bar{X}_{L}, \bar{X}_{L}^{\prime}\right)=P_{\mu}\left(\bar{X}_{n_{1}} \leq \bar{X}_{L}\right)+\int_{\bar{X}_{L}}^{\infty} f_{\mu}(x) P_{\mu}\left(\bar{X}_{n_{1}+n_{2}} \leq \bar{X}_{L}^{\prime}\right) d x \\
=P_{\mu}\left(\bar{X}_{n_{1}} \leq \bar{X}_{L}\right)+\int_{\bar{X}_{L}}^{\infty} f_{\mu}(x) P_{\mu}\left(\bar{X}_{n_{2}} \leq \frac{\bar{X}_{L}^{\prime}\left(n_{1}+n_{2}\right)-x n_{1}}{n_{2}}\right) d x \\
=\Phi\left(\frac{\bar{X}_{L}-\mu}{\sigma / \sqrt{n_{1}}}\right) \\
+\int_{\bar{X}_{L}}^{\infty} \frac{1}{\sqrt{2 \pi \sigma^{2} / n_{1}}} \exp \left(-\frac{(x-\mu)^{2}}{\frac{2 \sigma^{2}}{n_{1}}}\right) \Phi\left(\frac{\frac{\bar{X}_{L}^{\prime}\left(n_{1}+n_{2}\right)-x n_{1}}{n_{2}}-\mu}{\sigma / \sqrt{n_{2}}}\right) d x
\end{aligned}
$$

In equation (6), $f_{\mu}(x)$ is the probability density function (PDF) of $\bar{X}_{n_{1}}$ when the true

mean level of efficacy is $\mu . \bar{X}_{L}$ was derived from $P_{L}$ as described above. $\bar{X}_{L}^{\prime}$ was defined as the low boundary for the sample mean to claim a positive trial from $N_{\max }$ patients, which was obtained based on posterior probability. Although this is slightly different from Lee and Liu's design, we obtain almost identical results if we assume the predictive probability of enrolling future patients is 0 when we reach $N_{\max }$ by the end of second stage. Equation (6) consists two parts. The first part is the probability of stopping the trial at the end of the first stage due to futility, and second part is the probability of failing to reject the null at the end of the second stage. Thus, the overall type I error $(\alpha)$ and type II error rate $(\beta)$ can be expressed as

$$
\alpha=1-\varphi\left(\mu_{0}, \sigma, n_{1}, n_{2}, \bar{X}_{n_{1}}, \bar{X}_{L}, \bar{X}_{L}^{\prime}\right)
$$




$$
\beta=\varphi\left(\mu_{1}, \sigma, n_{1}, n_{2}, \bar{X}_{n_{1}}, \bar{X}_{L}, \bar{X}_{L}^{\prime}\right)
$$

The probability of early termination, $\operatorname{PET}\left(\mu_{0}\right)$, at the end of the first stage under the null hypothesis is

$$
\operatorname{PET}\left(\mu_{0}\right)=P_{\mu}\left(\bar{X}_{n_{1}} \leq \bar{X}_{L} \mid H_{0}\right)
$$

The expected sample size under the null hypothesis $E\left(N \mid \mu_{0}\right)$ is

$$
E\left(N \mid \mu_{0}\right)=n_{1}+\left(1-\operatorname{PET}\left(\mu_{0}\right)\right) * n_{2}
$$

\subsubsection{Optimization Criteria}

Although our proposed two-stage PP design is based on Bayesian predictive probability framework, we wish to only consider designs that satisfy both type I and type II error rates constraints. Similar to those proposed by Simon [19], we use optimal design that minimizes the expected sample size, $E\left(N \mid \mu_{0}\right)$, under the null hypothesis, while minimax design that minimizes the maximum sample size.

To find the optimal and minimax design, the following parameters should be pre-specified:

- Maximum uninteresting mean and minimum interesting mean: $\mu_{0}$ and $\mu_{1}$

- Likelihood variance: $\sigma^{2}$

- Prior distribution for mean $\mu: N\left(\theta, \tau^{2}\right)$

- Lower and upper boundaries of predictive probability: $P_{L}$ and $P_{U}$

- Threshold posterior probability: $P_{T}$

- Nominal type I and type II error rate constraint: $\alpha$ and $\beta$ 
$P_{L}, P_{U}$ and $P_{T}$ should be between 0 and 1 . When we want to stop the trial early only for futility, we chose $P_{U}=1.0$ not to allow early stopping for efficacy since there are situations when the treatment is beneficial for patients, there is little reason to stop the trial early. In addition, it will help gain more precision for the estimation of clinical endpoints of interest and provide more information for future large trials. $P_{T}$ is usually a large positive constant between 0 and 1, in this paper, we set the searching range for $P_{T}$ as [0.9 - 0.99]. $P_{L}$ is usually a small positive constant, which we set as $[0.001-0.1]$ for the searching range in this study. How to choose $P_{T}$ and $P_{L}$ as well as their impact stopping rules will be discussed in Section 4. By searching over all possible values of in the range of $P_{L}$ and $P_{T}$, the goal is to identify the combinations of $P_{L}$ and $P_{T}$ to yield the desirable power within the constraint of the specified type I error rates. The sample size from single-stage trial for continuous variables was used to derive the searching range for $N_{\max }$. Although a bit arbitrary, if we denote $N$ as the sample size required for the single-stage design, we used the range from $0.7 \mathrm{~N}$ to $1.7 \mathrm{~N}$ to search for $N_{\max }$. According to our simulation experiences, this range covers almost all the situations. Therefore, by varying $n_{1}$ and $N_{\max }$ from small to large, the design with the smallest expected sample size under the null hypothesis, that controls both type I and type II error rates at the nominal level, is defined the optimal design. Similarly, the design with the smallest $N_{\max }$ is defined as the minimax design.

\subsection{Results}

Table 2.1 and Table 2.2 illustrate both optimal and minimax two-stage designs which only allows early termination for futility. The tabulated results vary by effect size $(1,0.8,0.5$ and 0.2$)$. The effect size, also referred as to Cohen's $\mathrm{d}$, is defined as $\left(\mu_{1}-\right.$ 
$\left.\mu_{0}\right) / \sigma$, where $\mu_{0}$ and $\mu_{1}$ are the average tumor size change under null and alternative hypothesis, respectively [68]. We chose $0.2,0.5,0.8$ and 1 to cover the range of possibilities of small, medium and large effect sizes. We also provided other effect sizes from 0.1 to 0.9 in the appendices (Table A3.1-3.4). Table 2.1 and Table 2.2 display $P_{L}, P_{T}$, total sample size from two-stages $N_{\max }$, sample size for the first stage $n_{1}$, the upper boundary of the reject region at the end of the first stage $\bar{X}_{L}$ and the upper boundary of the reject region at the end of the second stage $\bar{X}_{L}^{\prime}$. Following Lee and Liu's definition, the reject region was the outcome space leading to the rejection of the new treatment/agent. The probability of early termination (PET), expected total sample size under the null hypothesis $\left(E_{n}\right)$ and actual type I and II error rates are also reported.

In this scenario, we assume an average of $20 \%$ tumor size shrinkage after standard treatment or from historical control, and we wish to test if the new treatment or agent will improve the shrinkage to $30 \%$. Thus, the null hypothesis is $H_{0}: \mu \leq 20 \%$ and the alternative hypothesis is $H_{A}: \mu \geq 30 \%$, where $\mu_{0}=20$ and $\mu_{1}=30$. Positive values of the parameter favor the new drug efficacy, whereas negative values represent the disease progression. We chose various $\sigma(10,12.5,20$ and 50) to compare the sample size for different effect sizes, where in practice, $\sigma$ should be obtained from historical trials or estimated from standard treatment. We set ESS $=\frac{\sigma^{2}}{\tau^{2}}=1$ and $\theta=\mu_{0}=20$. As described above, the searching range for $N_{\max }$ was obtained from a single-stage trial with continuous endpoints. A grid search was performed through the space of $P_{L}[0.001-0.1]$ and $P_{T}[0.9$ - 0.99] to enumerate all the combinations that meet pre-specified type I and type II error constraints. 
For instance, in Table 2.1, when $\sigma=20$ with effect size 0.5 , type I and type II error rate constraints are 0.05 and 0.2 , a grid search of $P_{L}$ and $P_{T}$ was performed for each $N_{\max }$ between 8 and 46. It turns out that the design with 11 patients in the first stage and 26 patients in total is the optimal design, which has the smallest expected sample under the null hypothesis. $P_{L}$ and $P_{T}$ are determined to be 0.1 and 0.945 , respectively. The trial will be stopped, and the new drug is considered not promising for further study when the average tumor size shrinkage from the first 11 patients is less than $20.69 \%$. If the observed value of the mean of tumor size shrinkage from the first 11 patients is greater than $20.69 \%$, the trial should continue to the second stage and enroll an additional 15 patients. The expected total sample size is 17.8 , and the probability of early termination is 0.55 when the true mean tumor size change is $20 \%$. Alternatively, the minimax design requires 25 patients in total with 18 patients in the first stage (Table 2.2). In fact, the minimax design usually gives a smaller total sample size but larger $n_{1}, \bar{X}_{n_{1}}, E_{n}$ and PET. This trend is consistent with both Simon's and Hsiao's designs $[19,56]$. As expected, the total sample size required decreases as effect size increases.

Table 2.3 and 2.4 display the optimal designs and minimax designs, which allow early termination due to either futility or efficacy. In both designs, we use $P_{U}=0.95$ to make the efficacy stopping decision. Using the same example, when $\sigma=20$ with effect size 0.5 , type I and type II error rate constraints are 0.05 and 0.2 , the optimal design yields the same total sample 26 with 12 patients required for the first stage (Table 2.3). The trial will be stopped and the new drug is considered not promising for further study when the average tumor size shrinkage from the first 11 patients is less than $21.20 \%$. If the observed value of the mean of tumor size shrinkage from the first 11 patients is greater than $33.86 \%$, 
the trial should be stopped early for efficacy. Otherwise, an additional 14 patients will be enrolled for the $2^{\text {nd }}$ stage. The corresponding operating characteristics are very similar to the futility design. That is because we choose $P_{U}=0.95$, which is close to $P_{U}=1$. As the $P_{U}$ decreases, the total sample size increases.

\subsubsection{Roles of priors}

As mentioned above, ESS can be interpreted as the amount of information contained in the prior. In the case of normal distribution, ESS is $\sigma^{2} / \tau^{2}$. To investigate the roles of normal conjugate priors in this proposed design, we considered two groups of priors: "pessimistic" priors that set the mean of normal prior equal the maximum uninteresting mean $\left(\theta=\mu_{0}\right)$ under the null hypothesis and the other group is for "optimistic" priors that set the prior mean equal to the minimum mean of interest $\left(\theta=\mu_{1}\right)$ under the alternative hypothesis. For each group, we assessed two priors, one "weaker" prior with ESS $=1$ and one "stronger" with ESS $=5$. For purposes of illustration, we still use effect size $=0.5$ as an example with $\mu_{0}=20$ and $\mu_{1}=30$.Thus, four priors were used as follows:

- Weak pessimistic prior: $N\left(20,20^{2}\right), \mathrm{ESS}=\sigma^{2} / \tau^{2}=1$

- $\quad$ Strong pessimistic prior: $N\left(20,8.94^{2}\right), \mathrm{ESS}=\sigma^{2} / \tau^{2}=5$

- Weak optimistic prior: $N\left(30,20^{2}\right), \mathrm{ESS}=\sigma^{2} / \tau^{2}=1$

- $\quad$ Strong optimistic prior: $N\left(30,8.94^{2}\right), \mathrm{ESS}=\sigma^{2} / \tau^{2}=5$

The numeric results of both optimal and minimax design are shown in Table 2.5 and Table 2.6. For the four optimal designs under different priors (Table 2.5), the total sample size is the same as 26 . Under two stronger priors $N\left(20,8.94^{2}\right)$ and $N\left(30,8.94^{2}\right)$, 
the strong optimistic prior $\mathrm{N}\left(30,8.94^{2}\right)$ results in a larger expected sample size of 18.97 under the null hypothesis, compared to 17.32 from the strong pessimistic prior $N\left(20,8.94^{2}\right)$. It is intuitive since the trial is less likely to be terminated early due to futility under the null hypothesis if the optimistic prior belief is true ( $\mathrm{PET}=0.58$ for prior $N\left(20,8.94^{2}\right)$ vs. PET $=0.54$ for prior $\left.N\left(30,8.94^{2}\right)\right)$. Under two weaker priors $N\left(20,20^{2}\right)$ and $N\left(30,20^{2}\right)$, the PET is the same but the optimistic prior also leads to a larger expected sample size of (18.25 vs. 17.81) under the null hypothesis. When comparing the minimax design (Table 2.6), all the four priors resulted in the sample maximum sample size ( $N=$ 25). The two weaker priors need a larger sample size from the first stage $\mathrm{n}_{1}$ than the stronger priors.

\subsubsection{Case Study}

In this section, we refer to the case study illustrated in Wason et al. [5]. In this case study, the primary endpoint was the maximum shrinkage in total lesion diameter and was previously classified as a binary variable according to RECIST criteria. Thus, the trial was previously designed using a Simon's two-stage design. In their proposed two-stage design, Wason et al. demonstrated that using continuous endpoints, instead of classified binary endpoints, there was about 50\% reduction in both expected and maximum sample size. According to their notation, the mean treatment efficacy (true tumor shrinkage) was denoted by $\delta$. The hypothesis testing framework was $H_{0}: \mu \leq \mu_{0}$ vs. $H_{1}: \mu \geq \mu_{1}$, where $\mu_{0}=-7.6$ and $\mu_{1}=11.1$, with known $\sigma=36.4$. Under this setup, Wason's optimal continuous design yielded a first and second stage sample size of 15 and 24, respectively, which compared with Simon's two-stage optimal design (30 patients are required for the 
first stage and 52 patients were required for the second stage), led to around $50 \%$ reduction in the sample size for both stages.

We apply our Bayesian PP design for this trial scenario. In particular, $\mu_{0}=-7.6$, $\mu_{1}=11.1$, and $\sigma=36.4$. We chose the prior as $N\left(-7.6,36.4^{2}\right)$ and set the same type I and II error rates as 0.05 and 0.1 , respectively. The searching range for $P_{L}$ is [0.001 - 0.1] and for $P_{T}$ is [0.9- 0.99]. These values result in the optimal continuous design having the first stage sample size of 14 and total sample size of 34 . The expected sample size under the null is 23.4. The cut-off value for tumor size change from the first 14 patients is -6.86 , with $P_{T}=0.945$ and $P_{L}=0.099$ (Table 2.7). The result is very similar to Wason et al., but requires a slightly smaller sample size in the second stage.

\subsection{Discussion}

In practice, RECIST criteria is commonly applied to categorize the tumor shrinkage into complete response, partial response, progressive disease, and stable disease. Response rate, which is the combination of complete and partial response, with at least a $30 \%$ decrease in the sum of the longest diameter of the target lesions, is usually used as a secondary endpoint in most of the cancer trials. However, as shown in the previous studies, this dichotomization not only lead to loss of statistical efficiency, but greatly increases the required sample size $[5,54,56]$.

In this proposed design, we extended Lee and Liu's predictive probability approach into a two-stage setting for continuous endpoint. Similar as Wason et al., we obtained decreased sample size when treating tumor shrinkage as continuous variable. The design parameters $P_{L}, P_{U}$, and $P_{T}$ plays an important role in searching for the optimal and 
minimax designs. As mentioned above, we chose $P_{U}=1$ to only allow for early futility stopping. Regarding the choice of $P_{L}, P_{U}$, and $P_{T}$, no definitive and clear guidelines have been proposed for the binary endpoints. We adopted similar strategy as described in Lee and Liu's paper and Dong et al's research [37, 39]. We set the range of $P_{T}$ for searching as [0.9 - 0.99]. $P_{T}$ can be viewed as a reasonable degree of certainty, which could be 0.9 , 0.95 and 0.99 , according to FDA guidelines for the use Bayesian statistics in medical device clinical trials $[39,69]$. The lower boundary for $\theta_{T}$ can be reduced to 0.8 , as Lee and Liu did in their binary PP design. In general, as $P_{T}$ increases, it is harder to reject $H_{0}$. Therefore, the higher $\theta_{T}$ is, the lower power and actual type I error it will be. There is no general guideline for choosing $P_{L}$. Lee and Liu set the search range for $P_{L}$ as [0.001-0.1], while Dong et al. used values up to $P_{L}=0.5$ [39]. We applied the same rule as Lee and Liu, since a smaller value of $P_{L}$ indicates that it is very unlikely the mean tumor size change will be larger than $\mu_{0}$ given current information, which turns into harder rejection of $H_{0}$ due to futility as well as increased power and type I error rates.

We also assessed the operating characteristics for the optimal designs based on the posterior approach. Our simulation results have shown that a two-stage design based on the posterior approach is more conservative, and always yields a little bit larger total sample size $N_{\max }$ and larger $\bar{X}_{n_{1}}$ (data not shown). For instance, if we use posterior probability approach for the case study, the $\bar{X}_{n_{1}}$ is -2.59 , and we need 39 patients in total with 15 patients for the first stage, which happened to be the same as Wason's design. However, as mentioned in Lee and Liu's paper, predictive probability approach has the advantages that it mimics the decision making process in reality and more intuitive [37]. 
We explored and evaluated the roles of priors for this PP design. In practice, prior information is usually obtained from standard treatment or historical control. ESS represents the weight we place on the prior information known before the trial. In this paper, we began with ESS = 1, assuming a weak prior. The total sample size remains robust with stronger priors $(\mathrm{ESS}=5)$ for both optimal and minimax designs. The comparison is illustrative but not conclusive. Based on our simulation, ESS should not exceed 1/3 of the total sample size. Future work on the exploration of other non-conjugated priors is needed.

There are several limitations to this current design that we plan to address in future work. Firstly, in this proposed design, we assume the standard deviation $\sigma$ is known in line with Wason's design. It is reasonable since in practice, this value could be obtained from previous trials or preclinical studies. However, when there is little prior information available, especially for novel drugs or biomarkers, unknown variance may be explored in the future. Secondly, this design currently accommodates only one interim look for futility, and we plan to eventually incorporate continuously monitoring for both futility and superiority. Further, priors are important in Bayesian clinical trial designs. It is always challenging and controversial to choose a suitable prior on the parameter of interest. In this paper, we only chose the conjugate priors for the mean, assuming variance is known, and explored briefly on the roles of pessimistic and optimistic priors. There could be clinical situations where the prior does not follow a normal distribution, and thus the resulting posterior does not have a closed form. More exploration is needed in this regard.

Overall, we have shown a two-stage design using predictive probability for continuous endpoints. We have demonstrated similar sample size reduction as Wason's design. We developed $\mathrm{R}$ programs to perform this proposed design and the code will be 
available in an upcoming $\mathrm{R}$ package, but these functions are available upon request. As research rapidly moves to incorporate more immunotherapies and targeted therapies, these designs will accommodate new types of outcomes while allowing for flexible stopping rules for futility and/or efficacy to continue optimizing trial resources and prioritizing agents with compelling early phase data. 
Figure 2.1 Proposed two-stage PP design for continuous endpoints.

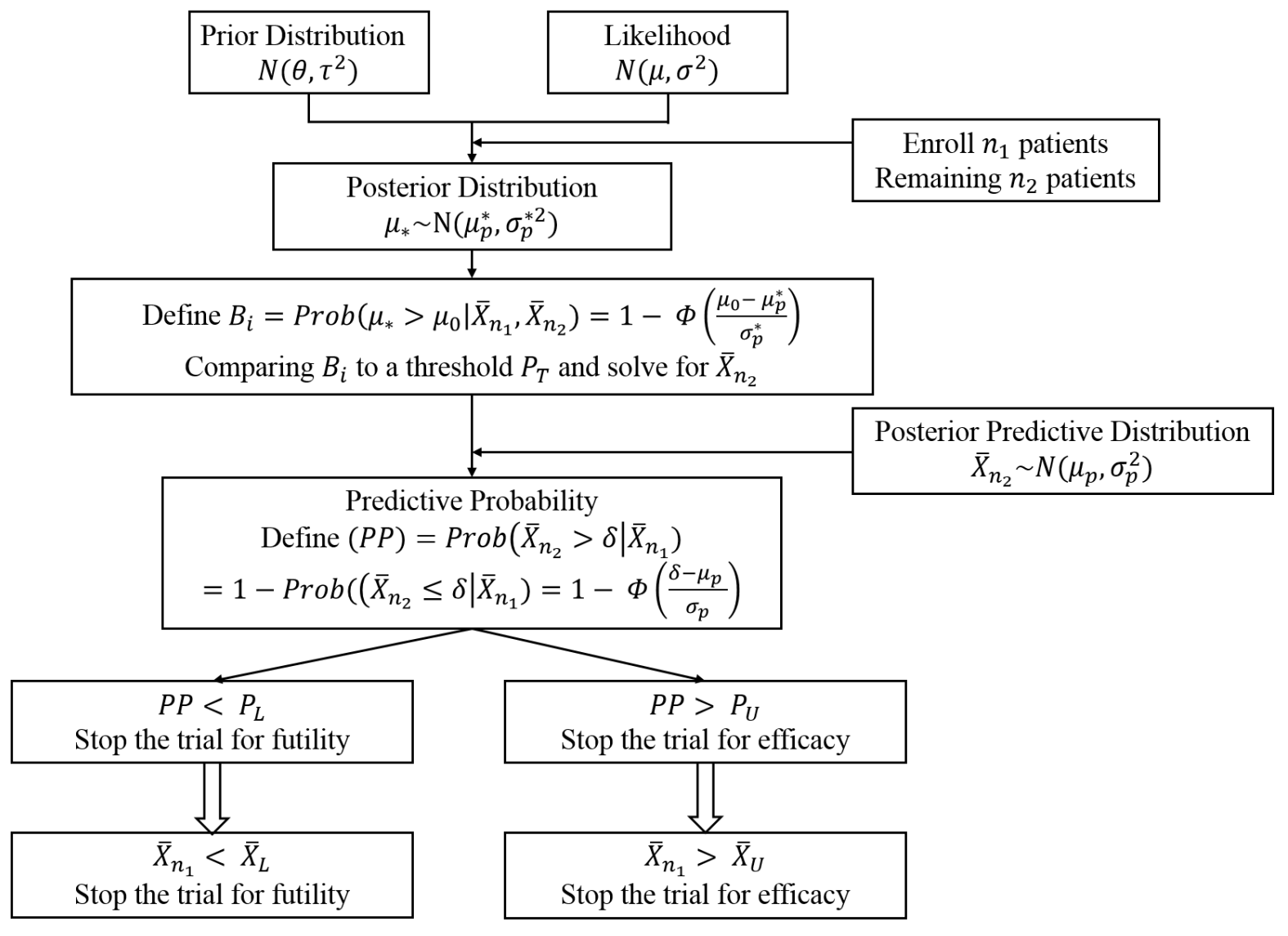


Table 2.1 Operating characteristics of optimal design for continuous endpoints with early termination for futility. For both designs below, $\mu_{0}=20, \mu_{1}=30, \theta=\mu_{0}=20, \sigma=10,12.5,20$ and 50, $\tau=\sigma$. The first row for each effect size is with type I and type II error rate constraints $(\alpha, \beta)=(0.05,0.2)$ and the second row for each effect size is with $(\alpha, \beta)=(0.05,0.1)$.

Optimal Design

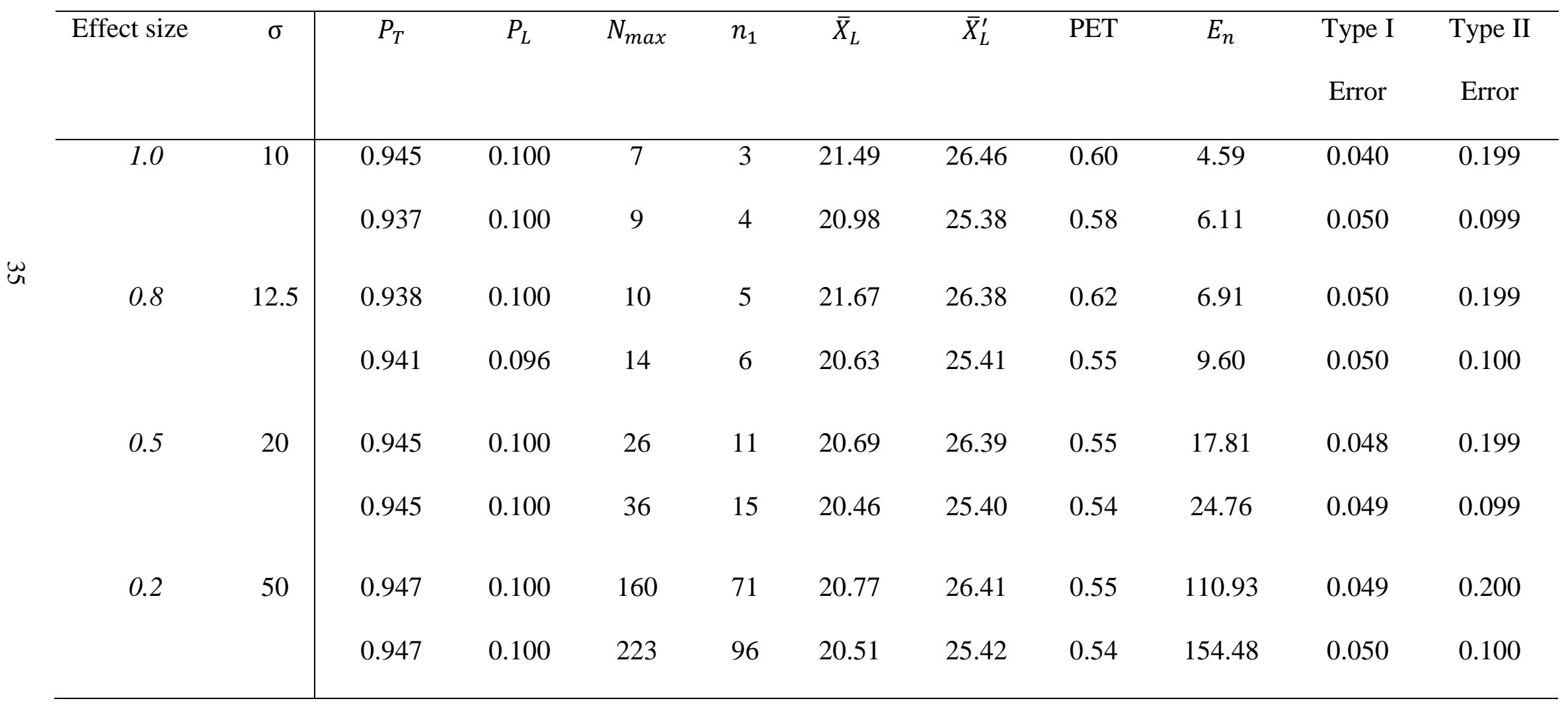


Table 2.2 Operating characteristics of minimax design for continuous endpoints with early termination for futility. For both designs below, $\mu_{0}=20, \mu_{1}=30, \theta=\mu_{0}=20, \sigma=10,12.5,20$ and 50, $\tau=\sigma$. The first row for each effect size is with type I and type II error rate constraints $(\alpha, \beta)=(0.05,0.2)$ and the second row for each effect size is with $(\alpha, \beta)=(0.05,0.1)$.

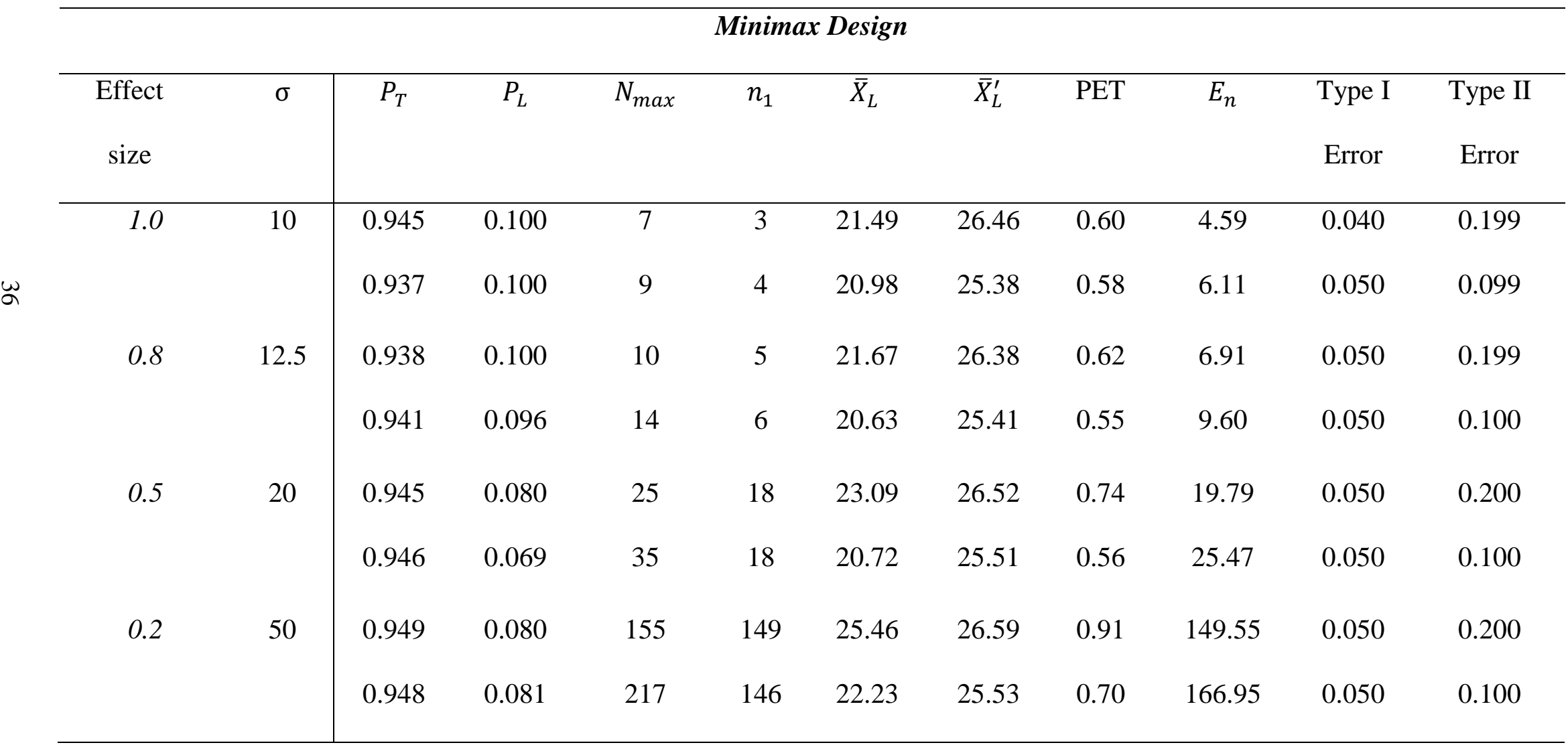


Table 2.3 Operating characteristics of optimal design for continuous endpoints with early termination for efficacy. For both designs below, $P_{U}=0.95, \mu_{0}=20, \mu_{1}=30, \theta=\mu_{0}=20, \sigma=10,12.5,20$ and 50, $\tau=\sigma$. The first row for each effect size is with type I and type II error rate constraints $(\alpha, \beta)=(0.05,0.2)$ and the second row for each effect size is with $(\alpha, \beta)=(0.05,0.1)$.

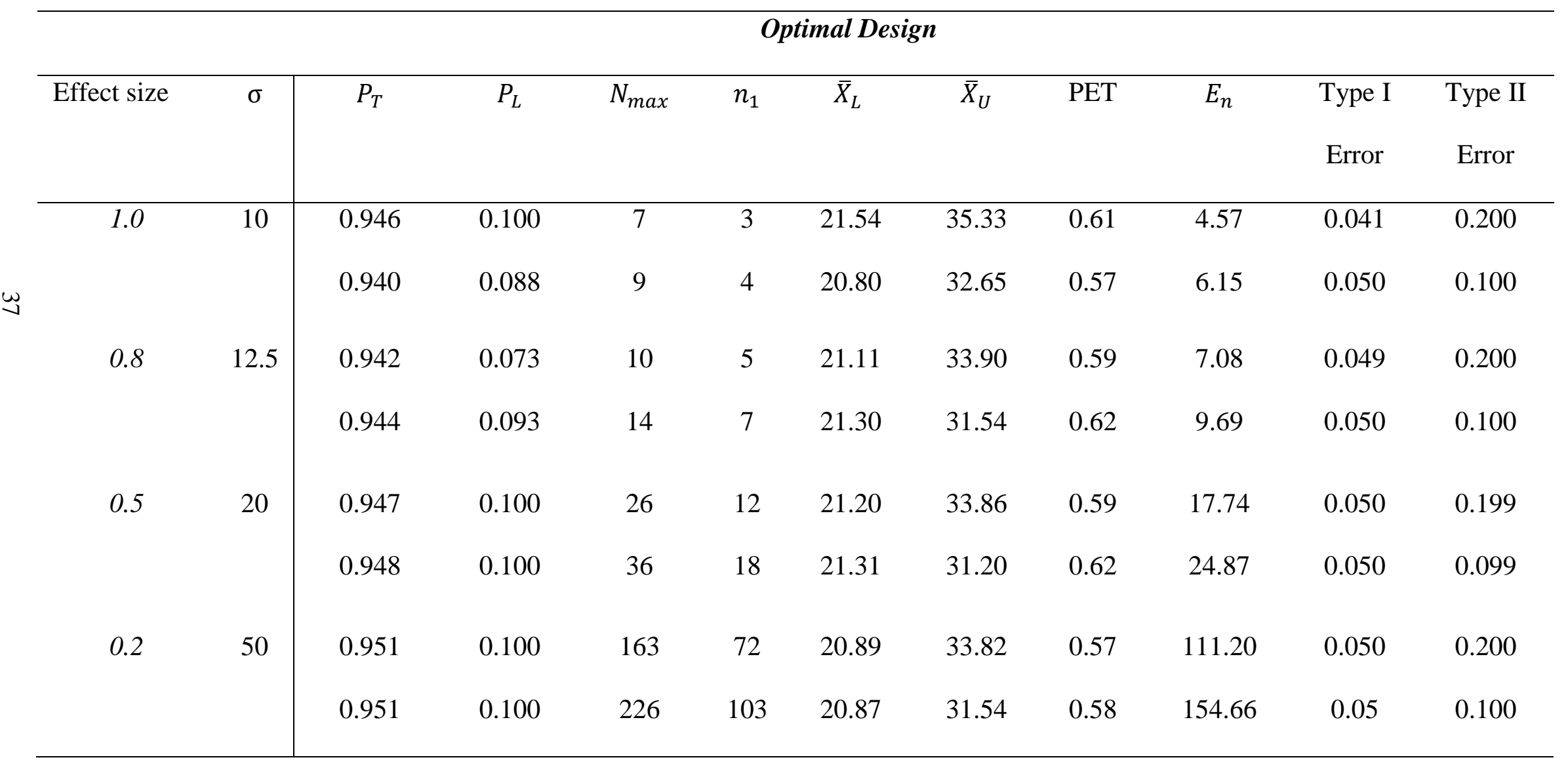


Table 2.4 Operating characteristics of minimax design for continuous endpoints with early termination for efficacy. For both designs below, $P_{U}=0.95, \mu_{0}=20, \mu_{1}=30, \theta=\mu_{0}=20, \sigma=10,12.5,20$ and $50, \tau=\sigma$. The first row for each effect size is with type I and type II error rate constraints $(\alpha, \beta)=(0.05,0.2)$ and the second row for each effect size is with $(\alpha, \beta)=(0.05,0.1)$.

Minimax Design

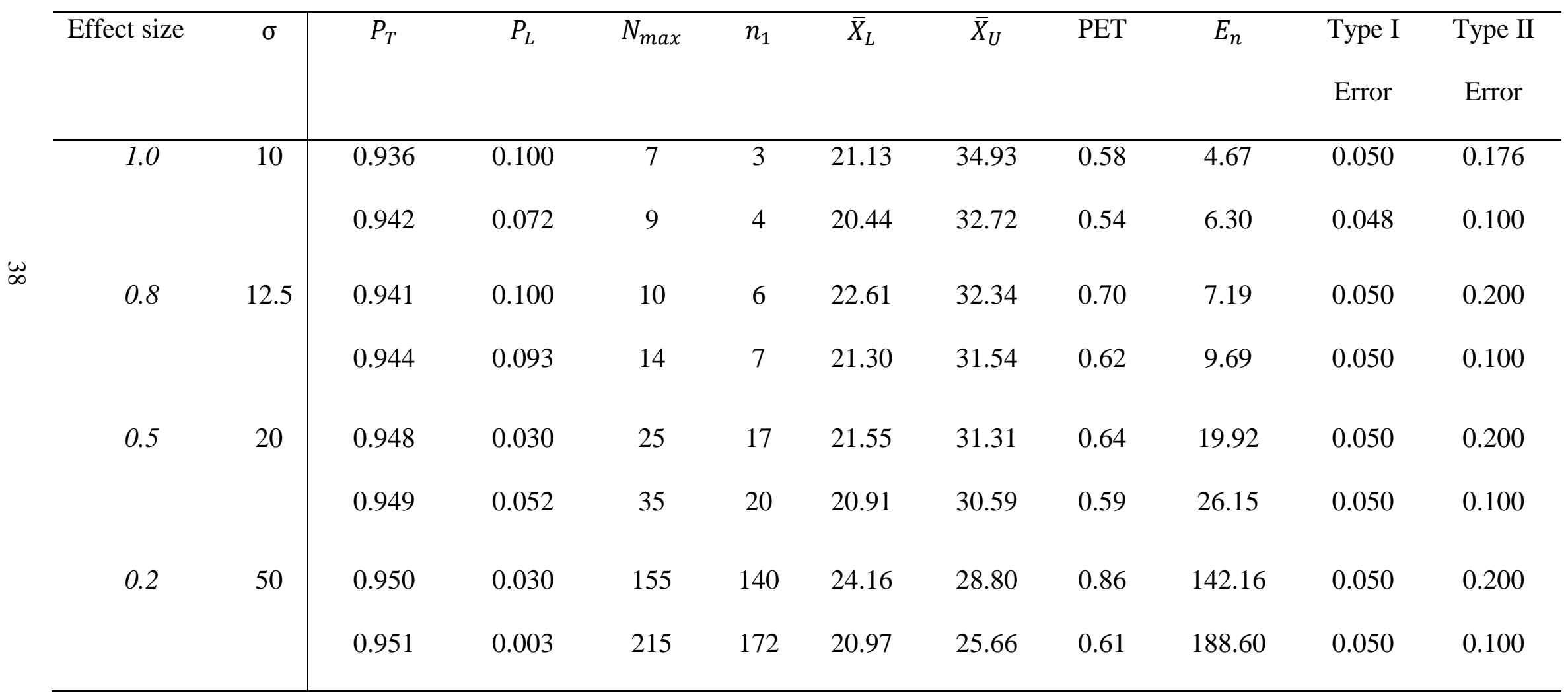


Table 2.5 Operating characteristics of optimal futility designs with different priors. (Design parameter: $\mu_{0}=20, \mu_{1}=30, \alpha=0.05$, $\beta=0.2, \sigma=20$ )

\begin{tabular}{l|cccccccccc}
\hline $\begin{array}{c}\text { Prior } \\
\mathrm{N}\left(\theta, \tau^{2}\right)\end{array}$ & $P_{T}$ & $P_{L}$ & $N_{\max }$ & $n_{1}$ & $\bar{X}_{L}$ & $\bar{X}_{L}^{\prime}$ & PET & $E_{n}$ & $\begin{array}{c}\text { Type I } \\
\text { Error }\end{array}$ & $\begin{array}{c}\text { Type II } \\
\text { Error }\end{array}$ \\
\hline$N\left(20,20^{2}\right)$ & 0.945 & 0.100 & 26 & 11 & 20.69 & 26.39 & 0.55 & 17.81 & 0.048 \\
$N\left(20,8.94^{2}\right)$ & 0.930 & 0.099 & 26 & 11 & 21.20 & 26.32 & 0.58 & 17.32 & 0.049 \\
$N\left(30,20^{2}\right)$ & 0.957 & 0.100 & 26 & 12 & 20.78 & 26.48 & 0.55 & 18.25 & 0.047 & 0.200 \\
$N\left(30,8.94^{2}\right)$ & 0.976 & 0.100 & 26 & 13 & 20.57 & 26.55 & 0.54 & 18.97 & 0.046 & 0.199 \\
\hline
\end{tabular}

w Table 2.6 Operating characteristics of minimax futility designs with different priors. (Design parameter: $\mu_{0}=20, \mu_{1}=30, \sigma=$ $20, \alpha=0.05, \beta=0.2$ )

\begin{tabular}{l|cccccccccc}
\hline $\begin{array}{c}\text { Prior } \\
\mathrm{N}\left(\theta, \tau^{2}\right)\end{array}$ & $P_{T}$ & $P_{L}$ & $N_{\max }$ & $n_{1}$ & $\bar{X}_{L}$ & $\bar{X}_{L}^{\prime}$ & PET & $E_{n}$ & $\begin{array}{c}\text { Type I } \\
\text { Error }\end{array}$ & $\begin{array}{c}\text { Type II } \\
\text { Error }\end{array}$ \\
\hline$N\left(20,20^{2}\right)$ & 0.945 & 0.081 & 25 & 18 & 23.10 & 26.52 & 0.74 & 19.79 & 0.050 & 0.200 \\
$N\left(20,8.94^{2}\right)$ & 0.932 & 0.039 & 25 & 13 & 20.26 & 26.53 & 0.52 & 18.77 & 0.050 & 0.200 \\
$N\left(30,20^{2}\right)$ & 0.955 & 0.079 & 25 & 16 & 22.16 & 26.52 & 0.67 & 19.00 & 0.050 & 0.200 \\
$N\left(30,8.94^{2}\right)$ & 0.975 & 0.047 & 25 & 15 & 20.44 & 26.59 & 0.53 & 19.66 & 0.049 & 0.200 \\
\hline
\end{tabular}


Table 2.7 Operating characteristics of optimal design and minimax design for case study. The searching range for $P_{T}$ is [0.9-0.99] and $P_{L}$ is $[0.001-0.1] . \mu_{0}=-7.6, \mu_{1}=11.1, \sigma=36.4 . \theta=\mu_{0}=-7.6, \tau=\sigma=36.4$ and $(\alpha, \beta)=(0.05,0.1)$.

\begin{tabular}{c|cccccccccc}
\hline & $P_{T}$ & $P_{L}$ & $N_{\max }$ & $n_{1}$ & $\bar{X}_{L}$ & $\bar{X}_{L}^{\prime}$ & PET & $E_{n}$ & $\begin{array}{c}\text { Type I } \\
\text { Error }\end{array}$ & $\begin{array}{c}\text { Type II } \\
\text { Error }\end{array}$ \\
& & & & & & & & & & \\
\hline Optimal Design & 0.945 & 0.099 & 34 & 14 & -6.86 & 2.52 & 0.53 & 23.40 & 0.049 & 0.100 \\
Minimax Design & 0.943 & 0.083 & 33 & 18 & -5.30 & 2.56 & 0.61 & 23.91 & 0.049 & 0.098 \\
\hline
\end{tabular}




\section{CHAPTER THREE}

\section{A Multi-Stage Predictive Probability Interim Design for Phase II Clinical Trials with Continuous Endpoints}

\subsection{Abstract}

Numerous phase II cancer clinical trial designs are based on binary response rate outcomes. The loss of information is particularly inefficient due to the dichotomization of tumor shrinkage according to RECIST criteria. In this paper, we extended Lee and Liu's probability predictive design for binary outcomes into a single-arm multi-stage setting for continuous endpoints, such as continuous tumor shrinkage in cancer trials or any other short-term response endpoints. We present the numeric simulation outputs for minimax designs with different prior distributions, while maintaining robust results. This predictive probability design is flexible due to its Bayesian properties. It keeps updating the information during the trial as the patients are continuously enrolled. In the meanwhile, it also controls the frequentist type I and type II error rates. We also developed R programs to assist with the study design.

Keywords: Multi-stage design; Predictive probability; Interim strategy; Continuous endpoints

\subsection{Introduction}

Phase II clinical trials aim to screen out those ineffective treatment or therapies, and identify those effective ones to move forward to larger confirmatory randomized phase III trials. As a result, phase II trials tend to have shorter study periods, smaller sample sizes, and endpoints that aim to be predictive to the definitive phase III trials. In cancer phase II 
trials, a commonly used endpoint is objective response rate, which is dichotomized from continuous tumor shrinkage according to RECIST [1].

Many designs have been proposed to target potential promising new therapies for phase II trials, among which at least one interim look is usually incorporated to utilize the information from the interim data that has already been obtained. In the setting of single treatment regimen or one-arm design, for the purpose of evaluating the efficacy, Gehan's design and Simon's two-stage design as well as Fleming's two-stage design are still the most widely used two-stage designs in practice [18-19,61].In some scenarios,three-stage design and multi-stage design are preferred to two-stage designs, since they are usually more efficient when compared to the two-stage design, especially when the effect size is small, and total sample size required is large. A multi-stage design is more efficient to terminate the trial early if the new therapy is not effective $[20,70]$. Chen extended Simon's two-stage design into a three-stage design and claimed that the average sample size reduction from two-stage to three-stage is about $10 \%$ when the null hypothesis is true [20]. Fleming proposed a multiple testing procedure for one-sample design to allow for early termination while maintaining the statistical properties. In the randomized setting, group sequential designs have been widely employed, with Pocock's design and O'Brien and Fleming's design as two commonly used group-sequential options[62, 63].

On the other hand, Bayesian clinical trial designs have gained popularity due to their flexibility to include sequential stoppings when compared with their frequentist counterparts. Thall and Simon introduced a class of phase II Bayesian clinical trial designs [28]. Tan and Machin proposed a two-stage design based on the posterior distribution [29]. Sambucini extended it into a two-stage design based on predictive distribution [30, 31]. 
Wang et al. and Dong et al. developed two Bayesian single-arm two-stage designs incorporating both frequentist and Bayesian error rates [34, 39]. In particular, Lee and Liu proposed a predictive probability design for single-arm trial with binary outcome to monitor the trial continuously[37].

Although this binary endpoint is intuitive to understand and widely adopted in practice, loss of information from the dichotomization cannot be avoided. In addition, with the development of molecular targeted therapies and immunotherapies, drugs can be still active and patients can benefit from those "minor response" or "mixed response", even though drugs do not lead to high-levels of tumor regression and do not meet the criteria of response rates $[6,71,72]$. Therefore, using continuous tumor shrinkage instead of the binary response rate has been proposed in previous studies [5, 53-56, 58]. However, these designs can be extended to any continuous outcomes, for example, biomarker assessment over time. In this paper, we extended Lee and Liu's predictive probability design into a single-arm setting with continuous outcome. This manuscript is organized into the following sections. Section 3.3 details our proposed design and trial decision rules. Section 3.4 displayed the numeric results from the simulation and case study. Section 3.5 is to discuss the strengths, limitations and future directions of current design.

\subsection{Methods}

\subsubsection{Notation and Proposed Design}

In this paper, we extend Lee and Liu's $P P$ design into a multi-stage design for continuous endpoints. The hypothesis testing framework is as follows:

$$
H_{0}: \mu \leq \mu_{0} \text { vs. } H_{1}: \mu \geq \mu_{1}
$$


where $\mu_{0}$ represents the maximum efficacy from the standard treatment or historical control, and $\mu_{1}$ represents a target efficacy threshold from the treatment or therapy being studied. This extended design also meets both pre-specified Type I and Type II error rate constraints.

For illustration purposes, we define "tumor size change" as the percentage decrease in the sum of the longest diameter of the target lesions compared to baseline for each patient, with positive values denoting tumor shrinkage [73]. We assume the average tumor shrinkage, $X$, follows a normal distribution $N\left(\mu, \sigma^{2}\right)$, with mean $\mu$ and known variance $\sigma^{2}$. We assume a normal conjugate prior distribution for $\mu, N\left(\theta, \tau^{2}\right)$, which represents the investigator's previous knowledge of the tumor size change from standard treatment or historical control. We use effective sample size for the prior (ESS), $\frac{\sigma^{2}}{\tau^{2}}$ for the normal case, to assign the amount of information for the prior [67, 73]. For the purpose of simulation, we set $\mathrm{ESS}=1$, assuming very little knowledge is known before the trial starts. We also compare the effect of different ESS specifications in the results section.

Here, we consider a single-arm $K$-stage design, where $K \leq N_{\max }$. At the end of $i^{t h}$ stage, the number of patients enrolled in total is denoted as $n_{i}$, where $i=1,2, \ldots, K$. We denote $\bar{X}_{n_{i}}$ as the average tumor shrinkage for the current $n_{i}$ patients. Therefore, given the information from these current $n_{i}$ patients, the posterior distribution of the mean tumor shrinkage $\mu$ follows a normal distribution, with mean $\frac{\sigma^{2} \theta}{n_{i} \tau^{2}+\sigma^{2}}+\frac{n_{i} \tau^{2}}{n_{i} \tau^{2}+\sigma^{2}} \bar{X}_{n_{i}}$ and variance $\frac{\tau^{2} \sigma^{2}}{n_{i} \tau^{2}+\sigma^{2}}$. Thus, the average tumor shrinkage from the future $m_{i}$ patients, where $m_{i}=$ $N_{\max }-n_{i}$, follows a normal distribution with mean $\mu_{p}^{*}$ and $\sigma_{p}^{* 2}$, where 


$$
\begin{gathered}
\mu_{p}^{*}=\frac{\sigma^{2} \theta}{\left(m_{i}+n_{i}\right) \tau^{2}+\sigma^{2}}+\frac{n_{i} \tau^{2}}{\left(m_{i}+n_{i}\right) \tau^{2}+\sigma^{2}} \bar{X}_{n_{i}}+\frac{m_{i} \tau^{2} \bar{X}_{m_{i}}}{\left(m_{i}+n_{i}\right) \tau^{2}+\sigma^{2}} \\
\sigma_{p}^{* 2}=\frac{\tau^{2} \sigma^{2}}{\left(m_{i}+n_{i}\right) \tau^{2}+\sigma^{2}}
\end{gathered}
$$

We define $B_{i}=\operatorname{Prob}\left(\mu_{*}>\mu_{0} \mid \bar{X}_{n_{i}}, \bar{X}_{m_{i}}\right)$ to measure the probability that the mean tumor shrinkage is larger than $\mu_{0}$, given the mean tumor size change in current $n_{i}$ patients and future $m_{i}$ patients. We can conclude the treatment is efficacious when $B_{i}>P_{T}$, where $P_{T}$ is the posterior critical value at each stage and is usually assumed to be greater than 0.8 .

$$
\begin{gathered}
B_{i}=\operatorname{Prob}\left(\mu_{*}>\mu_{0} \mid \bar{X}_{n_{i}}, \bar{X}_{m_{i}}\right)=1-\operatorname{Prob}\left(\mu_{*} \leq \mu_{0} \mid \bar{X}_{n_{i}}, \bar{X}_{m_{i}}\right) \\
=1-\Phi\left(\frac{\mu_{0}-\mu_{p}^{*}}{\sigma_{p}^{*}}\right)>P_{T}
\end{gathered}
$$

where $\Phi($.$) represents the cumulative density function (CDF) of the standard normal$ distribution. The above inequality (1) can be derived into an inequality of $\bar{X}_{m_{i}}$ regarding $P_{T}$.

$$
\bar{X}_{m_{i}}>\frac{\left(\left(n_{i}+m_{i}\right) \tau^{2}+\sigma^{2}\right)\left(\mu_{0}-\sigma_{p}^{*} \Phi^{-1}\left(1-P_{T}\right)\right)-\sigma^{2} \theta-\tau^{2} n_{i} \bar{X}_{n_{i}}}{m_{i} \tau^{2}}=\delta
$$

The posterior predictive distribution of tumor size change from the future $m$ patients also follows a normal distribution $N\left(\mu_{p}, \sigma_{p}^{2}\right)$, with $\mu_{p}=\frac{\sigma^{2} \theta}{n_{i} \tau^{2}+\sigma^{2}}+\frac{n_{i} \tau^{2}}{n_{i} \tau^{2}+\sigma^{2}} \bar{X}_{n_{i}}$ and $\sigma_{p}^{2}=$ $\frac{\sigma^{2}}{m_{i}}+\frac{\tau^{2} \sigma^{2}}{n_{i} \tau^{2}+\sigma^{2}}$

Based on all the information above, we can then define the predictive probability $(P P)$ Predictive probability $(P P)=\operatorname{Prob}\left(\bar{X}_{m_{i}}>\delta \mid \bar{X}_{n_{i}}\right)$

$$
=1-\operatorname{Prob}\left(\bar{X}_{m_{i}} \leq \delta \mid \bar{X}_{n_{i}}\right)=1-\Phi\left(\frac{\delta-\mu_{p}}{\sigma_{p}}\right)
$$


A high $P P$ means that given current data, the treatment is likely to be efficacious by the end of the study; whereas a low PP suggests that the treatment may not have sufficient activity. Therefore, we use $P P$ to make the decision during the interims in the trial.

\subsubsection{Trial Decision Rules}

For this proposed design, we have the flexibility to stop the trial early either due to futility and superiority. We use similar decision rules as those described in Lee and Liu's $P P$ design and our two-stage design $[37,73]$. For a K-stage design $\left(K<N_{\max }\right)$, we denote $P P_{i}$ as the predictive probability at the end of $i^{t h}$ stage $(i \leq K)$. We also denote $P_{L}$ and $P_{U}$ as the lower and upper boundaries for $P P_{i}$, assuming $P_{L}$ and $P_{U}$ are constant for each stage. $P_{L}$ and $P_{U}$ should be both between 0 and 1 , where $P_{L}$ is usually a small positive number less than 0.1 and $P_{U}$ is usually a large number greater than 0.9. By comparing $P P_{i}$ with $P_{L}$ and $P_{U}$ and solving for $\bar{X}_{n_{i}}$, we can obtain $\bar{X}_{L_{i}}$ and $\bar{X}_{U_{i}}$, which are defined as the lower and upper bounds for the sample mean from current $n_{i}$ patients. Therefore, for a $K$-stage design, the decision rule can be described in terms of $\bar{X}_{L_{i}}$ and $\bar{X}_{U_{i}}$ at each stage:

- If $\bar{X}_{n_{i}}<\bar{X}_{L_{i}}$, stop the trial for futility and reject the alternative hypothesis;

- $\quad$ If $\bar{X}_{n_{i}}>\bar{X}_{U_{i}}$, stop the trial for efficacy and reject the null hypothesis;

- $\quad$ Otherwise, if $\bar{X}_{L_{i}} \leq \bar{X}_{n_{i}} \leq \bar{X}_{U_{i}}$, continue to the next stage.

\subsubsection{Frequentist Operating Characteristics}

As we want our design to meet both pre-specified type I and type II error rate constraints, we need to calculate the probability of rejecting the null hypothesis at each stage. Therefore, for the design described above, we obtain the average tumor shrinkage from the cumulative sample size of the patients at the end of each stage $i$, denoted as $\bar{X}_{n_{i}}$, where $\bar{X}_{n_{i}}$ follows a normal distribution with mean $\mu$ and variance $\sigma^{2} / n_{i}$. If we 
standardize $\bar{X}_{n_{i}}$ and transform it into a test statistics, $\mathrm{Z}_{n_{i}}=\left(\bar{X}_{n_{i}}-\mu_{0}\right) /\left(\sqrt{\sigma^{2} / n_{i}}\right)$, for a given design that continues to the end, the vector of $\mathrm{Z}=\left(\mathrm{Z}_{n_{1}}, \mathrm{Z}_{n_{2}}, \ldots, \mathrm{Z}_{n_{K}}\right)$ is multivariate normally distributed with the mean $\left(\mu-\mu_{0}\right) /\left(\sqrt{\sigma^{2} / n_{i}}\right)$ and variance 1 . The covariance between $\mathrm{Z}_{n_{i}}$ and $\mathrm{Z}_{n_{j}}$ is $\sqrt{\frac{n_{i}}{n_{j}}}$ for $n_{i}<n_{j}[74]$. Therefore, the vector of the $\bar{X}=$ $\left(\bar{X}_{n_{1}}, \bar{X}_{n_{2}}, \ldots, \bar{X}_{n_{K}}\right)$, is assumed to follow a multivariate normal distribution, with mean $\mu$ and variance $\frac{\sigma^{2}}{n_{i}}(i=1,2, \ldots, K)$. The covariance between $\bar{X}_{n_{i}}$ and $\bar{X}_{n_{j}}$, with $n_{i}<n_{j}$, is $\sigma^{2} / n_{j}$. The probability of rejecting $H_{0}$ with the true mean $\mu$ at each stage can be expressed as:

$$
\begin{aligned}
& \operatorname{Pr}\left(\text { reject the } H_{0} \text { at stage } i\right)
\end{aligned}
$$

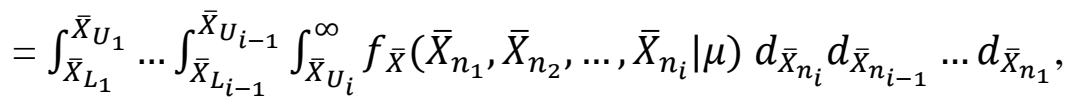

where $f_{\bar{X}}$ is the probability density function (pdf) of the multivariate normal distribution with mean vector and covariance matrix as previously described. $\bar{X}_{U_{i}}$ and $\bar{X}_{L_{i}}$ are boundaries obtained from predictive probabilities $P_{L}$ and $P_{U}$ at each stage $i$. When the trial continues to the last stage $K, \bar{X}_{U_{K}}$ and $\bar{X}_{L_{K}}$ are obtained from the posterior probability. In the two-stage paper, we compare the results with the scenario, in which we assume the predictive probability of enrolling future patients is 0 when we reach $N_{\max }$. It has been shown to be very similar in the two scenarios. Therefore, the type I error and power can be calculated by summing up the probability of rejecting the null hypothesis at each stage when the null and alternative hypothesis is true, respectively [55].

To evaluate the multidimensional integral in the equation above, wo strategies are applied. One directly integrates the multivariate normal density $[75,76]$. Computation time 
for the direct numeric integration is much less when the design only contains 3 or fewer stages. Thus, we utilize a published $\mathrm{R}$ package, which contains a function to perform a Monte Carlo algorithm and evaluate the integrand for 4 or more desired stages, as the computation time reduces significantly [77]. The actual type I error can be calculated by summing up (4) from each stage $i$ when $\mu=\mu_{0}$, with its value under $\mu=\mu_{1}$ yielding the power. One limitation for the current $\mathrm{R}$ package we utilized to evaluate the multidimensional integral is that only up to 40 stages is allowed due to their algorithm used in the package.

\subsubsection{Optimization Criteria}

This current proposed design is based on predictive probability and, similar to the two-stage design, we wish our design to meet both pre-specified type I and type II error rates constraints. In general, $P_{L}$ is usually chosen as a very small number, eg. less than or equal to 0.1 , while $P_{U}$ is usually chosen as a very large number, eg. greater than or equal to 0.9 . Both Lee and Liu's study and Dong et al. have suggested guidelines for the searching range of these parameter $[37,39]$. In this design, we restrict $P_{L}$ as 0.1 and $P_{U}$ as 0.95 , instead of searching $P_{L}$ and $P_{U}$ in a range, across all the stages in current manuscript, due to the grid search strategy. As the number of parameters increases, the computation time increases quickly. On the other hand, we set the searching range for $P_{T}$ to be [0.90 - 0.99]. In the two-stage paper, we already discussed how to choose $P_{T}$ and $P_{L}$ and their impact on stopping rules [73]. By searching through the range of $P_{T}$, we can identify the design that will yield desirable power within the constraint of the specified type I error rates. To determine the searching range for $N_{\max }$, we use the 0.8 to 1.5 times of the sample size from the single-stage design for continuous variables as the searching boundaries. In this paper, 
we only consider the minimax design here, which is to minimize the maximum sample size [19]. If there are more than one design with the same total sample size, we select the design with largest power.

To find the minimax design, the following parameters must be pre-specified:

- The number of stages in total $K$

- The proportions of patients enrolled at each interim look

- Maximum uninteresting mean and minimum interesting mean: $\mu_{0}$ and $\mu_{1}$

- Likelihood variance: $\sigma^{2}$

- Prior distribution for mean $\mu: N\left(\theta, \tau^{2}\right)$

- Lower and upper boundaries of predictive probability: $P_{L}$ and $P_{U}$

- Threshold posterior probability: $P_{T}$

- Nominal type I and type II error rate constraints: $\alpha$ and $\beta$

\subsection{Results}

We present the simulation results for 3-stage to 10-stage design for this proposed minimax design in Table 3.1 and Table A3.1. We assume the sample size at each stage is approximately equally spaced. We also tested effect sizes of $1,0.8,0.5$ and 0.2 (also referred as Cohen's d), representing the ranges of large, medium and small effect sizes. We assume the prior mean $\theta$ equals to the likelihood mean $\mu$ and we set the ESS as 1, assuming very little information is known before the trial starts. Table 3.1 shows the results satisfying the pre-specified type I error rate of 0.05 and type II error rate of 0.2 , while Table A3.1 shows the results meeting the pre-specified type I error rate of 0.05 and type II error of 0.1 . 
In the presented simulation scenario, we assume an average of $20 \%$ tumor size shrinkage after standard treatment or historical control. Thus, the null hypothesis is $H_{0} \leq$ $20 \%$. We expect our new therapy to improve this tumor shrinkage to at least $30 \%$. Thus, the alternative hypothesis is $H_{1} \geq 30 \%$. Several observations were made according to the simulation results. Firstly, we initially search $0.7-1.7$ times the sample size from onestage single-arm design for $N_{\max }$. It covers most of the situations according to our simulation experiences. However, if there were no combinations of the parameters that meet both type I and type II error constraints within this searching range, we need to extend the searching range for $N_{\max }$. Secondly, as the number of stages increases, with the same effect size, the number of smallest sample size required slightly increases. From 3-stage design to 10 -stage design, the total sample size increased by over $20 \%$. This increment is even higher when type II error rate constraint is 0.1 . Further, all the $P_{T}$ were determined to be in the middle of the searching range [0.9 -0.99$]$. From our experience, as $P_{T}$ increases, the type I error rates decrease while the type II error rates increase (data not shown).

\subsubsection{Roles of priors}

Similar to our two-stage paper, we explored both pessimistic and optimistic priors and assessed their effect on the design operating characteristics. The four priors are listed below:

- Weak pessimistic prior: $N\left(20,20^{2}\right), \mathrm{ESS}=\sigma^{2} / \tau^{2}=1$

- Strong pessimistic prior: $N\left(20,8.94^{2}\right), \mathrm{ESS}=\sigma^{2} / \tau^{2}=5$

- Weak optimistic prior: $N\left(30,20^{2}\right)$, ESS $=\sigma^{2} / \tau^{2}=1$

- Strong optimistic prior: $N\left(30,8.94^{2}\right), \mathrm{ESS}=\frac{\sigma^{2}}{\tau^{2}}=5$,

where "weak" denotes ESS = 1 and "strong" denotes ESS = 5, while "pessimistic" means $\theta=\mu_{0}$ and "optimistic" means $\theta=\mu_{1}$. The numeric results are presented in Table 3.2, 
and the stopping boundaries for the ten-stage design is shown in the Figure 3.1. It has been shown that the results are fairly robust. All four priors gave a similar smallest sample size required. It is interesting that the $P_{T}$ that leads to the minimax design is more similar with the same prior. When we use pessimistic priors, $P_{T}$ for the strong prior is smaller than the weak prior; while when we use optimistic priors, $P_{T}$ for the strong prior is larger than the weak prior. The stopping boundaries are getting closer to each other after the $3^{\text {rd }}$ interim look, which is intuitive in practice since the more information obtained, the more precise evidence we can apply to make our trial decision. We don't suggest make a decision too early based on very limited information.

\subsubsection{Case Study}

In this section, we apply this multi-stage design to the same unpublished trial illustrated in Wason et al. [5]. In this unpublished trial, the primary endpoint was a binary response rate and was originally designed as a Simon's two-stage design. In Wason's design, they transformed the binary endpoints into continuous variable using a standard normal distribution transformation (shown in the appendix). Therefore, $\mu_{0}=-7.6$ and $\mu_{1}=11.1$, with known $\sigma=36.4$. Under this setup, Wason's optimal continuous design yielded around $50 \%$ reduction in total sample size when compared to the design using binary response rate.

We apply our Bayesian PP design for this trial scenario. In particular, $\mu_{0}=-7.6$, $\mu_{1}=11.1$ and $\sigma=36.4$. We chose the prior as $N\left(-7.6,36.4^{2}\right)$ and set the same type I and II error rates as 0.05 and 0.1 , respectively. We set $P_{L}=0.1, P_{U}=0.95$ and searching range for $P_{T}$ to be $[0.9-0.99]$. We tested our design in two settings. Setting 1: equally spaced with 5 stages; Setting 2: equally spaced with 10 stages. The numeric results are 
presented below in Table 3.3, and the stopping boundaries are shown in Figure 3.2. The minimax design yields a total sample size for the 5-stage design of 40, with $P_{T}$ determined to be 0.953 . When the number of stages increases to 10 , the minimal total sample size required to meet both type I and type II error rates increases to 46 . The stopping boundaries for both settings are shown in figure 3.2. For example, in terms of 5-stage design, after 8 patients are enrolled at the end of first stage, if the average tumor shrinkage is greater than 22.95 we should stop the trial early for efficacy. If the average tumor shrinkage is less than -12.34 , we should stop the trial early for futility, otherwise trial should continue to the second stage.

We also assess the operating characteristics when the interim looks are not equally spaced. The numeric results are shown in Table 3.4. The first row in Table 3.2 is the equally spaced design. The second row is for stopping the trial after approximately $10 \%, 30 \%, 50 \%$, $70 \%$ and $100 \%$ of the patients are enrolled. The third row is to stop the trial after approximately $10 \%, 20 \%, 30 \%, 40 \%, 100 \%$ of the patients are enrolled. The last row is to stop the trial after approximately $60 \%, 70 \%, 80 \%, 90 \%$ and $100 \%$ of the patients are enrolled. In these four scenarios, $P_{T}$ is quite similar, meaning it may not affect the design when the searching range was given. However, the one with the first interim look at $60 \%$ of the patients are enrolled yields the smallest total sample, while the one with the first 4 interim looks before $50 \%$ of the patients are enrolled gives the largest total sample size.

\subsection{Discussion}

A group-sequential approach in clinical trials is usually to analyze the interim data after groups of patients were enrolled. It is usually more flexible and efficient than the 
design with fixed sample size. In this paper, we extended Lee and Liu's PP design for binary outcomes and proposed a multi-stage design for continuous endpoints based on predictive probability. The current design allows to stop the trial early either due to futility or efficacy, while maintaining the frequentist operating characteristics at the same time.

If continuous monitoring of the trial is desired, information from a certain number of patients after the trial starts is needed for the first interim analysis. Lee and Liu suggest the $1^{\text {st }}$ interim look should happen after $10 \%$ of the total patients are enrolled [37]. As we show in the case study, as more patients are analyzed in earlier stages, fewer total patients are required. In addition, when investigators choose equal sample size for each stage, when the effect size is large, the sample size at each stage may not be exactly the same due to rounding precision. For example, in Table 1, when the effect size equals to 1 and number of stages is 8 . The total sample size required is 11 , with 1 in each stage for the first 7 stages and 4 in the last stage. However, this situation may not be ideal and reasonable in practice. We would rather set larger percentage at the beginning of the trial. For example, regarding the design with 8-stage design and effect size of 1 , if we put 3 patients in the first stage and then 1 for each from stage 2 to stage 8 , the total sample size of 10 yields the desired minimax design.

There are several limitations in this current proposed design. Firstly, in this paper, we only assessed equal spaced interim looks from 3- to 10-stages. Although our R code can accommodate user-defined unequal spaced interim looks, we did not compare the operating characteristics from those unequally spaced interim looks with those design scenarios presented, except the case study in this manuscript. Therefore, one of the future directions is to explore how to space the interim looks during the whole trial to achieve the 
most desirable design, and how it will affect the design properties. Secondly, due to the current algorithm, we can only accommodate up to 40 interim looks during the trial due to the dependency of the R2Cuba package [77]. We plan to extend it into 'real' continuous monitoring in the future, although we need to determine how it is realistic in practice to monitor the trial continuously. In practice, the necessity of continuous monitoring depends on several factors, such as effect size, the overall sample size required, the timing of endpoints collection during the trial as well as trial resources and staffing. Thirdly, we only explored optimistic and pessimistic conjugated normal priors. Therefore, it would be interesting to assess how non-conjugated priors, both pessimistic and optimistic, will impact the results. Further, as we mentioned above in the manuscript, our grid searching strategy can only accommodate the minimax design due to computation time. It will be interesting to see how the probability of early termination under the null hypothesis and compare the expected sample size from the optimal design with the smallest sample size desired. We plan to optimize the code and incorporate it into our ongoing $\mathrm{R}$ package.

In general, we have shown the results from a multi-stage design using predictive probability for continuous endpoints. We have shown that in a two-stage design, the total sample size reduced nearly by half when we used the continuous endpoints, instead of dichotomizing them into binary endpoints. When it was extended to a multi-stage design, the total sample required increases as the number of interim looks increases, which is common to other group sequential designs. However, the multi-stage design provides more flexibility and statistical efficiency when compared to the two-stage design. We developed $\mathrm{R}$ functions to perform this proposed design and the code will be available in an upcoming $\mathrm{R}$ package, but these functions are currently available upon request. As research rapidly 
moves to incorporate more immunotherapies and targeted therapies, these designs will accommodate new types of outcomes while allowing for flexible stopping rules for futility and/or efficacy to continue to optimize trial resources and prioritize agents with compelling early phase data. 
Table 3.1 Operating characteristics of minimax multi-stage design for continuous endpoints. For both designs below, $\mu_{0}=20, \quad \mu_{1}=30, \theta=\mu_{0}=20, \sigma=$ $10,12.5,20$ and $50, \tau=\sigma . P_{L}=0.1$ and $P_{U}=0.95$.

\begin{tabular}{|c|c|c|c|c|c|c|}
\hline \multicolumn{7}{|c|}{$\alpha=0.05, \beta=0.2$} \\
\hline Effect size & $\sigma$ & $K$ & $P_{T}$ & $N_{\max }$ & Type I Error & Type II Error \\
\hline \multirow[t]{8}{*}{1.0} & 10 & 3 & 0.936 & 7 & 0.049 & 0.179 \\
\hline & & 4 & 0.936 & 8 & 0.049 & 0.159 \\
\hline & & 5 & 0.937 & 9 & 0.050 & 0.132 \\
\hline & & 6 & 0.940 & 11 & 0.049 & 0.087 \\
\hline & & 7 & 0.933 & 8 & 0.050 & 0.187 \\
\hline & & 8 & 0.940 & 11 & 0.047 & 0.141 \\
\hline & & 9 & 0.942 & 9 & 0.036 & 0.183 \\
\hline & & 10 & 0.931 & 10 & 0.046 & 0.093 \\
\hline \multirow[t]{8}{*}{0.8} & 12.5 & 3 & 0.942 & 12 & 0.050 & 0.156 \\
\hline & & 4 & 0.940 & 11 & 0.050 & 0.194 \\
\hline & & 5 & 0.942 & 13 & 0.050 & 0.147 \\
\hline & & 6 & 0.943 & 13 & 0.049 & 0.165 \\
\hline & & 7 & 0.942 & 13 & 0.049 & 0.165 \\
\hline & & 8 & 0.942 & 15 & 0.050 & 0.138 \\
\hline & & 9 & 0.941 & 17 & 0.047 & 0.126 \\
\hline & & 10 & 0.930 & 10 & 0.044 & 0.155 \\
\hline
\end{tabular}


Table 3.1 (Continued)

\begin{tabular}{cc|ccccc}
\hline \multicolumn{6}{c}{$\alpha=0.05, \beta=0.2$} \\
\hline Effect size & $\sigma$ & $K$ & $P_{T}$ & $N_{\max }$ & Type I Error & Type II Error \\
\hline 0.5 & 20 & 3 & 0.948 & 28 & 0.050 & 0.185 \\
& & 0.950 & 29 & 0.049 & 0.191 \\
& 5 & 0.951 & 30 & 0.049 & 0.193 \\
& 6 & 0.952 & 33 & 0.049 & 0.171 \\
& 7 & 0.952 & 33 & 0.050 & 0.183 \\
& 8 & 0.952 & 33 & 0.050 & 0.179 \\
& & 0.952 & 32 & 0.049 & 0.190 \\
& & 10 & 0.950 & 34 & 0.048 & 0.125 \\
\hline 0.2 & 3 & 0.953 & 171 & 0.049 & 0.198 \\
& 4 & 0.955 & 179 & 0.050 & 0.197 \\
& 5 & 0.957 & 189 & 0.049 & 0.198 \\
& 6 & 0.959 & 196 & 0.050 & 0.198 \\
& 7 & 0.960 & 202 & 0.050 & 0.198 \\
& 8 & 0.964 & 206 & 0.048 & 0.192 \\
& 9 & 0.967 & 216 & 0.046 & 0.199 \\
& 10 & 0.961 & 213 & 0.048 & 0.176 \\
\hline
\end{tabular}


Table 3.2 Operating characteristics of minimax designs with different priors. (Design parameters: $\mu_{0}=20, \mu_{1}=30, \sigma=20, \alpha=0.05, \beta=0.2, P_{L}=0.1$ and $\left.P_{U}=0.95\right)$. For simulation in this table, the effect size were set as 0.5 .

\begin{tabular}{|c|c|c|c|c|c|}
\hline $\begin{array}{c}\text { Prior } \\
\mathrm{N}\left(\theta, \tau^{2}\right)\end{array}$ & $K$ & $P_{T}$ & $N_{\max }$ & Type I Error & Type II Error \\
\hline Pessimistic Weak & 3 & 0.948 & 28 & 0.050 & 0.185 \\
\hline \multirow[t]{7}{*}{$N\left(20,20^{2}\right)$} & 4 & 0.950 & 29 & 0.049 & 0.191 \\
\hline & 5 & 0.951 & 30 & 0.049 & 0.193 \\
\hline & 6 & 0.952 & 33 & 0.049 & 0.171 \\
\hline & 7 & 0.952 & 33 & 0.050 & 0.183 \\
\hline & 8 & 0.952 & 33 & 0.050 & 0.179 \\
\hline & 9 & 0.952 & 32 & 0.049 & 0.190 \\
\hline & 10 & 0.950 & 34 & 0.048 & 0.125 \\
\hline Optimistic Weak & 3 & 0.960 & 27 & 0.049 & 0.200 \\
\hline \multirow[t]{7}{*}{$N\left(30,20^{2}\right)$} & 4 & 0.962 & 29 & 0.050 & 0.196 \\
\hline & 5 & 0.964 & 30 & 0.049 & 0.195 \\
\hline & 6 & 0.966 & 30 & 0.049 & 0.200 \\
\hline & 7 & 0.966 & 32 & 0.049 & 0.187 \\
\hline & 8 & 0.969 & 32 & 0.048 & 0.198 \\
\hline & 9 & 0.970 & 33 & 0.048 & 0.199 \\
\hline & 10 & 0.966 & 37 & 0.045 & 0.176 \\
\hline
\end{tabular}


Table 3.2 (Continued)

\begin{tabular}{c|c|cccc}
\hline $\begin{array}{c}\text { Prior } \\
\mathrm{N}\left(\theta, \tau^{2}\right)\end{array}$ & $K$ & $P_{T}$ & $N_{\max }$ & Type I Error & Type II Error \\
\hline Pessimistic Strong & 3 & 0.930 & 27 & 0.050 & 0.178 \\
$N\left(20,8.94^{2}\right)$ & 4 & 0.929 & 28 & 0.050 & 0.196 \\
& 5 & 0.929 & 29 & 0.049 & 0.200 \\
& 6 & 0.929 & 30 & 0.049 & 0.199 \\
& 7 & 0.927 & 31 & 0.049 & 0.197 \\
& 8 & 0.924 & 32 & 0.050 & 0.192 \\
\hline Optimistic Strong & 3 & 0.921 & 30 & 0.050 & 0.186 \\
$N\left(30,8.94^{2}\right)$ & 10 & 0.912 & 30 & 0.048 & 0.104 \\
\hline & 5 & 0.978 & 27 & 0.048 & 0.197 \\
& 5 & 0.978 & 28 & 0.050 & 0.190 \\
& 6 & 0.980 & 29 & 0.049 & 0.189 \\
& 7 & 0.981 & 30 & 0.048 & 0.196 \\
& 8 & 0.981 & 31 & 0.048 & 0.189 \\
& 9 & 0.975 & 31 & 0.049 & 0.048 \\
& 10 & 0.978 & 31 & 0.047 & 0.162 \\
\hline
\end{tabular}


Figure 3.1: Stopping Boundaries for the 10-stage design with 4 different priors. In this scenario, the parameters are as follows: $\mu_{0}=$ $20, \mu_{1}=30, \sigma=20, P_{L}=0.1, P_{U}=0.95$, searching range for $P_{T}$ is $[0.9,0.99]$. All the four designs meet the pre-specified type I and type II error rates $(\alpha, \beta)=(0.05,0.2)$.

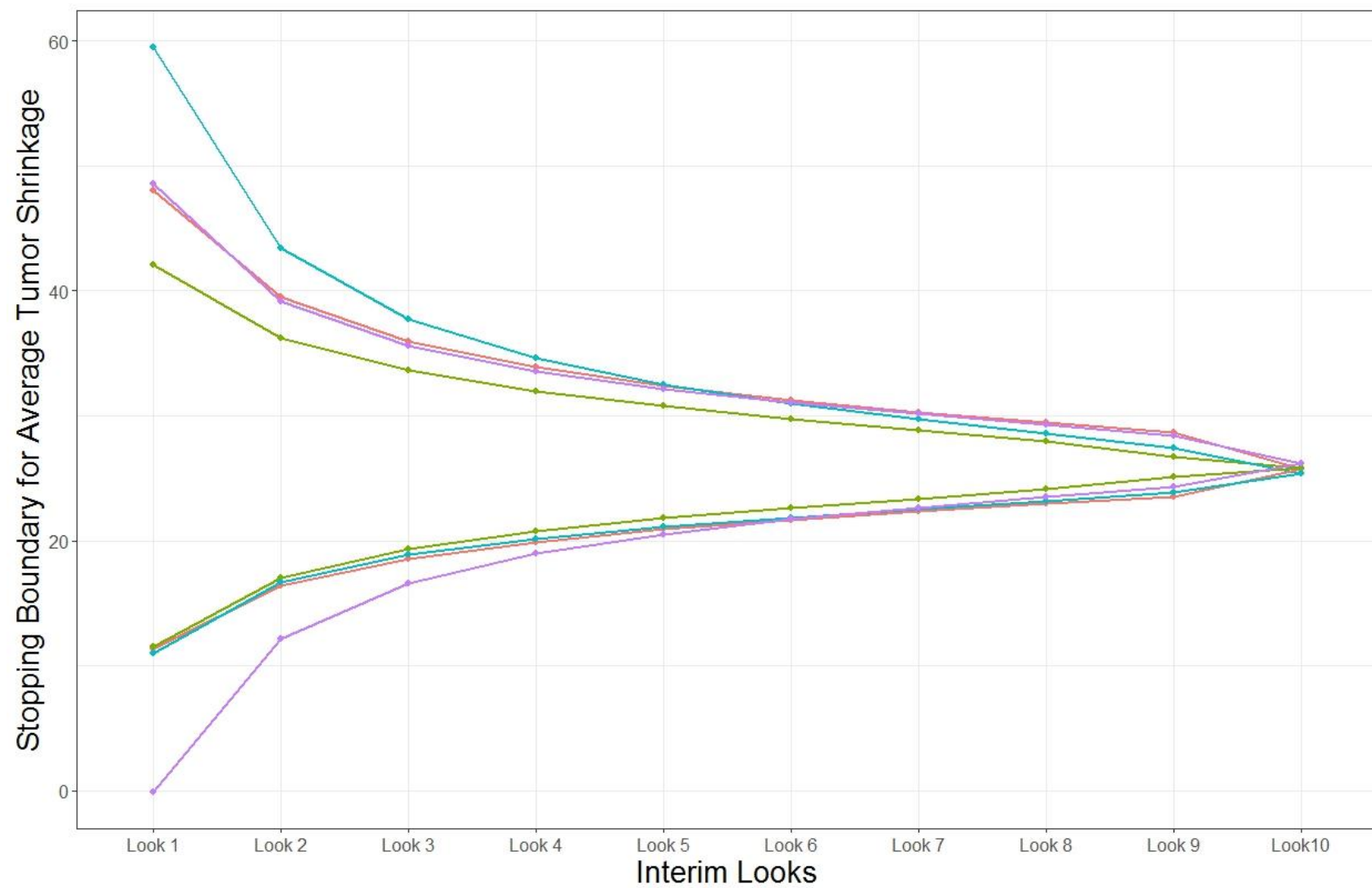

$\rightarrow \mathrm{N}\left(20,20^{\wedge} 2\right)$ - Weak Pessimistic

- N(30, 20^2) - Weak Optimistic

$-\mathrm{N}\left(20,8.94^{\wedge} 2\right)$ - Strong Pessimistic

$\rightarrow \mathrm{N}\left(30,8.94^{\wedge} 2\right)$ - Strong Optimistic 
Table 3.3 Operating characteristics of 5-stage and 10-stage Minimax design with equal space for case study. The searching range for $\mathrm{P}_{\mathrm{T}}$ is [0.9-0.99]. $\mathrm{P}_{\mathrm{L}}=0.1$ and $\mathrm{P}_{\mathrm{U}}=0.95 . \mu_{0}=-7.6, \mu_{1}=11.1, \sigma=36.4 . \theta=\mu_{0}=-7.6, \tau=\sigma=36.4$ and $(\alpha, \beta)=(0.05,0.1)$.

\begin{tabular}{c|cccc}
\hline & $P_{T}$ & Nmax & Type I Error & Type II Error \\
& & & & \\
\hline 5-stage design & 0.953 & 40 & 0.049 & 0.096 \\
10-stage design & 0.954 & 46 & 0.047 & 0.097 \\
\hline
\end{tabular}

Table 3.4 Operating characteristics of 5-stage unequal space for case study. The searching range for $\mathrm{P}_{\mathrm{T}}$ is $[0.9-0.99]$. $\mathrm{P}_{\mathrm{L}}=0.1$ and $\mathrm{P}_{\mathrm{U}}=$ 0.95. $\mu_{0}=-7.6, \mu_{1}=11.1, \sigma=36.4 . \theta=\mu_{0}=-7.6, \tau=\sigma=36.4$ and $(\alpha, \beta)=(0.05,0.1)$.

9

\begin{tabular}{ccccc|crrr}
\hline$N_{1}$ & $N_{2}$ & $N_{3}$ & $N_{4}$ & $N_{5}$ & $P_{T}$ & Type I Error & Type II Error \\
\hline 8 & 16 & 24 & 32 & 40 & 0.953 & 0.049 & 0.096 \\
5 & 13 & 22 & 31 & 43 & 0.955 & 0.050 & 0.098 \\
5 & 9 & 14 & 18 & 45 & 0.958 & 0.050 & 0.100 \\
22 & 26 & 29 & 33 & 36 & 0.950 & 0.047 & 0.093 \\
\hline
\end{tabular}


Figure 3.2: Stopping Boundaries for the 5-stage design and 10-stage design for case study.
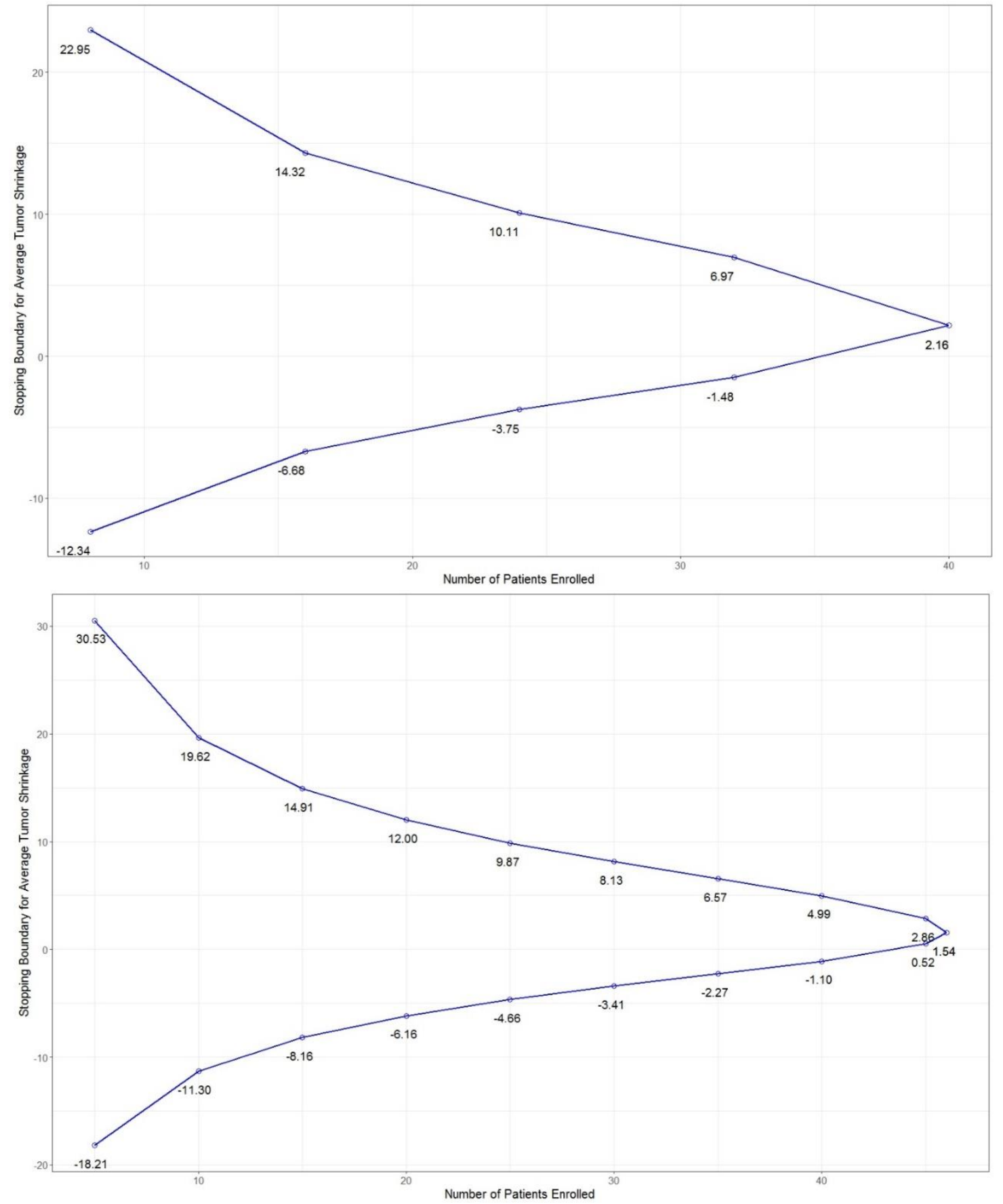


\section{CHAPTER FOUR}

\section{PPSC: An R Package for Single-Arm Phase II Predictive Probability Design with}

Continuous Endpoints

\subsection{Abstract}

In practice, interim analyses are usually incorporated into a trial to stop the trial early either due to futility and/or superiority. Early stopping of the trial can prevent patient exposure to ineffective therapies or accelerate drug development if beneficial effects are observed. In the Bayesian framework, interim designs based on predictive probability provide a flexible way to monitor the trial continuously while incorporating the evidence from the current data and making inference for the future. We extended a predictive probability design for binary outcomes into a single-arm trial setting for continuous outcomes and provide both optimal and minimax designs. In this paper, we describe the usage and implementation of the R package PPSC, which aims to implement a grid search strategy to find the optimal and minimax designs for continuous outcomes based on predictive probability. The package PPSC contains two functions and provides the solutions for both two-stage and multi-stage designs. These two functions give clinical investigators and biostatisticians the flexibility to monitor the trial and provide the stopping boundaries for futility and efficacy when early stopping the trial is desired.

Keywords: predictive probability; continuous outcome; interim design; phase II; R.

\subsection{Introduction}

A conventional phase II trial aims to evaluate the treatment effect of a drug or therapy being studied and identify the promising ones to carry on to the confirmative phase III trials. Interim strategies are of great importance in each stage of clinical trials. As 
developed for protecting participants from unsafe drugs or to hasten the general implementation of a beneficial therapy, interim strategies can be utilized to stop the trials early either for safety, futility or efficacy [14].

Many statistical methods have been developed for phase II trial designs, for both two-stage and multi-stage designs, regarding different types of endpoints. For binary endpoints, Gehan developed a two-stage design to stop the trial early due to futility if no response is observed from the first stage [18]. Simon proposed both optimal design and minimax design allowing one interim stop for futility [19]. Several extensions of Simon's design have been proposed [20, 21, 78]. Recent years, Bayesian approaches have been widely applied in the clinical trial designs. In particular, Tan and Machin proposed a twostage design based on posterior distribution of the true response rate and Sambucini extended it into predictive version [29-31]. Dong et al. suggested a two-stage design incorporating both frequentist and Bayesian error rates [39].

Regarding multi-stage designs, group-sequential designs are widely used in practice, in which groups of patients, either equally spaced or unequally spaced, are enrolled and the hypothesis is tested multiple times during accrual the trial. The most popular group sequential designs are O’Brian-Fleming' design and Pocock's design [62, 63]. Jennison ad Turnbull provide an thorough overview of group-sequential methodology [74]. Under Bayesian framework, in particular, Lee and Liu developed a predictive probability design for the binary response rate to continuously monitor the trial [37].

We extended Lee and Liu's predictive probability design into a setting for continuous outcome. This paper introduces an R package, PPSC, which provides the implementation of our extended predictive probability designs. This package specifically 
incorporates two types of designs: the two-stage design and the multi-stage design. The two-stage design is primarily for the purpose of early stopping due to futility, while it also has the capacity to stop early due to efficacy. The multi-stage design allows early stopping of the trial due to either futility or efficacy, which can accommodate up to 40 stages with 39 interim looks.

The paper is organized as follows. Section 4.3 describes the both two-stage design and multi-stage design as well as the trial decision rules. Section 4.4 details the implementation of the two main functions in the package and gives a series of examples of how to use the options in the functions. Section 4.5 discusses the limitations and future directions for current design and package.

\subsection{Methods}

For both two-stage and multi-stage designs, the hypothesis testing framework is as follows:

$$
H_{0}: \mu \leq \mu_{0} \text { vs. } H_{1}: \mu \geq \mu_{1}
$$

where $\mu_{0}$ represents the maximum efficacy from the standard treatment or historical control, while $\mu_{1}$ represents a target efficacy threshold from the treatment or therapy being investigated. Both designs meet pre-specified Type I and Type II error rate constraints.

For illustration purposes, we use tumor shrinkage as an example of continuous outcomes. However, this design is not limited to tumor shrinkage. It could be used for changes in biomarkers, pre-post designs or any other continuous endpoints. We present this one example throughout for simplicity. Here, we define "tumor size change/tumor shrinkage" as the percentage decrease in the sum of the longest diameter of the target 
lesions compared to baseline for each patient, with positive values denoting tumor shrinkage. We assume the average tumor shrinkage, $X$, follows a normal distribution $N\left(\mu, \sigma^{2}\right)$, with mean $\mu$ and known variance $\sigma^{2}$. We assume a normal conjugate prior distribution for $\mu, N\left(\theta, \tau^{2}\right)$ and we use effective sample size for the prior (ESS), the ratio of likelihood variance to prior variance $\left(\frac{\sigma^{2}}{\tau^{2}}\right)$ for the normal case, to assign the amount of information for the prior [67]. For the purpose of simulation, we set ESS $=1$, assuming very little knowledge is known before the trial starts. In practice, ESS and priors should be input from the investigators and represent the investigator's previous knowledge of the tumor size change or endpoint being studied from standard treatment or historical control.

We generalized the notation for the two-stage design to be a special case of the multi-stage design. We consider a single-arm trial with a total sample size of $N_{\max }$ and total $K$ stages, where $K \leq N_{\max }$. When $\mathrm{K}=2$, it represents the two-stage design. At the end of $i^{t h}$ stage, the number of patients enrolled in total is denoted as $n_{i}$, where $i=$ $1,2, \ldots, K$. We denote the average tumor shrinkage for $n_{i}$ patients as $\bar{X}_{n_{i}}$. Therefore, given the information from these current $n_{i}$ patients, the posterior distribution of the mean tumor shrinkage $\mu$ follows a normal distribution, with mean $\frac{\sigma^{2} \theta}{n_{i} \tau^{2}+\sigma^{2}}+\frac{n_{i} \tau^{2}}{n_{i} \tau^{2}+\sigma^{2}} \bar{X}_{n_{i}}$ and variance $\frac{\tau^{2} \sigma^{2}}{n_{i} \tau^{2}+\sigma^{2}}$. Thus, the average tumor shrinkage from the future $m_{i}$ patients, where $m_{i}=$ $N_{\max }-n_{i}$, follows a normal distribution with mean $\mu_{p}^{*}$ and $\sigma_{p}^{* 2}$, where

$$
\begin{gathered}
\mu_{p}^{*}=\frac{\sigma^{2} \theta}{\left(m_{i}+n_{i}\right) \tau^{2}+\sigma^{2}}+\frac{n_{i} \tau^{2}}{\left(m_{i}+n_{i}\right) \tau^{2}+\sigma^{2}} \bar{X}_{n_{i}}+\frac{m_{i} \tau^{2} \bar{X}_{m_{i}}}{\left(m_{i}+n_{i}\right) \tau^{2}+\sigma^{2}} \\
\sigma_{p}^{* 2}=\frac{\tau^{2} \sigma^{2}}{\left(m_{i}+n_{i}\right) \tau^{2}+\sigma^{2}}
\end{gathered}
$$


We define $B_{i}=\operatorname{Prob}\left(\mu_{*}>\mu_{0} \mid \bar{X}_{n_{i}}, \bar{X}_{m_{i}}\right)$ to measure the probability that the mean tumor shrinkage is larger than $\mu_{0}$, given the mean tumor size change in current $n_{i}$ patients and future $m_{i}$ patients. We can conclude the treatment is efficacious when $B_{i}>P_{T}$, where $P_{T}$ is the posterior critical value at each stage and is usually assumed to be greater than 0.8 .

$$
\begin{gathered}
B_{i}=\operatorname{Prob}\left(\mu_{*}>\mu_{0} \mid \bar{X}_{n_{i}}, \bar{X}_{m_{i}}\right)=1-\operatorname{Prob}\left(\mu_{*} \leq \mu_{0} \mid \bar{X}_{n_{i}}, \bar{X}_{m_{i}}\right) \\
=1-\Phi\left(\frac{\mu_{0}-\mu_{p}^{*}}{\sigma_{p}^{*}}\right)>P_{T}
\end{gathered}
$$

where $\Phi(\cdot)$ represents the cumulative density function (CDF) of the standard normal distribution. The above inequality (1) can be derived into an inequality of $\bar{X}_{m_{i}}$ regarding $\theta_{T}$.

$$
\bar{X}_{m_{i}}>\frac{\left(\left(n_{i}+m_{i}\right) \tau^{2}+\sigma^{2}\right)\left(\mu_{0}-\sigma_{p}^{*} \Phi^{-1}\left(1-P_{T}\right)\right)-\sigma^{2} \theta-\tau^{2} n_{i} \bar{X}_{n_{i}}}{m_{i} \tau^{2}}=\delta
$$

The posterior predictive distribution of tumor size change from the future $m$ patients also follows a normal distribution $N\left(\mu_{p}, \sigma_{p}^{2}\right)$, with $\mu_{p}=\frac{\sigma^{2} \theta}{n_{i} \tau^{2}+\sigma^{2}}+\frac{n_{i} \tau^{2}}{n_{i} \tau^{2}+\sigma^{2}} \bar{X}_{n_{i}}$ and $\sigma_{p}^{2}=$ $\frac{\sigma^{2}}{m_{i}}+\frac{\tau^{2} \sigma^{2}}{n_{i} \tau^{2}+\sigma^{2}}$

Based on all the information above, we can then define the predictive probability $(P P)$

Predictive probability $(P P)=\operatorname{Prob}\left(\bar{X}_{m_{i}}>\delta \mid \bar{X}_{n_{i}}\right)$

$$
=1-\operatorname{Prob}\left(\bar{X}_{m_{i}} \leq \delta \mid \bar{X}_{n_{i}}\right)=1-\Phi\left(\frac{\delta-\mu_{p}}{\sigma_{p}}\right)
$$


A high $P P$ means that given current data, the treatment is likely to be efficacious by the end of the study; whereas a low PP suggests that the treatment may not have sufficient activity. Therefore, we use $P P$ to make the decision during the interims in the trial.

- If $P P_{i}<P_{L}$, stop the trial and reject the alternative hypothesis;

- If $P P_{i}>P_{U}$, stop the trial and reject the null hypothesis;

- Otherwise, continue to the next stage.

$P P_{i}$ can also be transformed into $\bar{X}_{n_{i}}$, and the trial decision rules can be made according to the futility and efficacy boundaries at the end of each stage.

Although our design stopping boundaries are based on Bayesian predictive probabilities, we additionally restrict our designs to satisfy pre-specified type I and type II error rates constraints. Therefore, we need to calculate the probability of rejecting the null hypothesis at each stage. The detailed calculations for type I error rates and power are documented in Chapter 2 and 3.

\subsection{Use of the package PPSC}

Package PPSC depends on three libraries: R2Cuba, cubature and ggplot2 [76, 77, 79]. Packages cubature and R2Cuba are employed to conduct the multidimensional integration either using direct numeric integration or Monte Carlo algorithm. When the design has more than three stages, integration using Monte Carlo algorithm is much faster than numeric integration. Package ggplot 2 is utilized to plot the design stopping boundaries. Both designs give clinical investigators and biostatisticians the flexibility to monitor the trial and stop the trial early when necessary. 
Package PPSC contains two functions, par2stage and parmultistage. Function par2stage conducts a single-arm PP design, which incorporates one interim look, allowing for early stopping only due to futility. Function parmultestage performs a single-arm PP design allows for early stopping due to both futility and superiority, and currently allows for up to 40 interim looks. Regardless of design selected, input options are described in Table 4.1 and Table 4.2.

For both functions, there are several arguments in common. To implement the two designs, the users should specify a couple of parameters: the likelihood standard deviation "sigma" $(\sigma)$; the mean "theta" $(\theta)$ and standard deviation "tau" $(\tau)$ for the normal conjugate priors, which represents the previous knowledge about the endpoint from the investigators; the null value "mu0" $\left(\mu_{0}\right)$ and alternative value "mu1" $\left(\mu_{1}\right)$ for the hypothesis testing; the low and high boundaries for the posterior probability $\left(P_{T}\right)$, "pTlow" and "pThigh", as well as the searching increment for $\left(P_{T}\right)$, "pTby". As defined in Lee and Liu's design, $P_{T}$ is usually a large number between $[0.8,1]$, while Dong et al. suggest a larger number such as $0.95[37,39]$. The default setting is "pTlow $=0.9$, pThigh $=0.99$ and $p$ Tby $=0.01$ ". For the two-stage design, users also need to specify the low and high boundaries for the predictive probability $\left(P_{L}\right)$, "pLlow" and "pLhigh", as well as the searching increment for $\left(P_{L}\right)$, "pLby". $P_{L}$ is usually a small number below 0.1 . The default setting is "pLlow $=0.01$, pLhigh $=0.1$ and pLby $=0.01$ ”. Although two-stage design is primarily for early termination due to futility, the trial can also stop early for efficacy. To do so, the user can specify the upper boundary for the predictive probability $\left(P_{U}\right)$, "pU", which is usually a large number greater than 0.9 . The default value is 0.95 . In addition, for the multi-stage design, users have the flexibility of specifying the following parameters: if the trial is 
continuously monitored "continuous", with the default value "F"; the number of interims looks involved in the trial "num.interim", with the default value "num.interim $=2$ " for the 3-stage design; if the number of patients at each stage is the same "equal.space", with default setting "equal.space $=\mathrm{T}$ " for the same number of patients at each stage. Further, for both designs, the users need to specify the type I and type II error rates constraints, "pre.typeIerror" and "pre.typeIIerror". The default settings are "pre.typeIerror $=0.05$ " and "pre.typeIIerror $=0.2$ ". The plot for stopping boundaries can be outputted with the argument "plot_yes" being "T".

Another argument for both function is "plot_yes", for which if the value is "T", the plot for stopping boundaries is outputted.

\subsubsection{Two-stage design for early stopping due to futility: use of par2stage}

\section{Example 1:}

Consider the situation where the standard therapy or historical control will lead the tumor shrinkage up to $20 \%$ shrinkage and the clinical investigators want to test if the new therapy will improve the tumor shrinkage to at least $30 \%$. The trial only allows for early stopping due to futility. Therefore, we set $\mathrm{pU}=1$. The likelihood standard deviation in this example is assumed to be 20 , which should be input from the investigator or from historical control data. If we assume little information is known before the trials starts, we use ESS $=1$. Thus, theta $=20$ and tau $=20$. The searching range for $\mathrm{pL}$ is to be $[0.01,0.1]$ and for $\mathrm{pT}$ is to be $[0.9,0.99]$. The increment for grid search is 0.01 for both $\mathrm{pT}$ and $\mathrm{pL}$. The prespecified type I error rate is set to 0.05 , and type II error rate is set to 0.2 . The $\mathrm{R}$ code necessary to run this scenario is: 
Example $1<$ - par2stage $($ sigma $=20$, tau $=20$, theta $=20, \operatorname{mu} 0=20$, mul $=30$, pTlow $=0.90$, pThigh $=0.99$, Thby $=0.01$, pLlow $=0.01, \mathrm{pLhigh}=0.1, \mathrm{pLby}=0.01, \mathrm{pU}$ $=1$, pre.typeIerror $=0.05$, pre.typeIIerror $=0.2$, plot_yes $=\mathrm{T}$ )

The output is a list containing two elements, which give the operating characteristics for both optimal design and minimax design. In terms of the optimal design, the total sample size required is 27 with 11 for the first stage. The futility boundaries for first and second stage are 20.681 and 26.447. The corresponding type I and type II error rates are 0.044 and 0.199 . The $\mathrm{pT}$ and $\mathrm{pL}$ that yield the optimal design are 0.95 and 0.1 . The minimax design yields a very similar parameter combination, with 26 as the total sample size, 14 as the sample size for the first stage, 21.048 and 26.575 as the futility boundaries.

\$Optimal Design`

[1]

Total Sample Size $\quad 27$

Sample Size for the First Stage $\quad 11$

Futility Boundaries at the End of the First Stage $\quad 20.681$

Futility Boundaries at the End of the Second Stage $\quad 26.447$

Probability of Early Termination $\quad 0.545$

Expected Sample Size under the null $\quad 18.281$

Type I Error $\quad 0.044$

Type II Error $\quad 0.199$

pT $\quad 0.950$

$\mathrm{pL} \quad 0.100$

[2] 


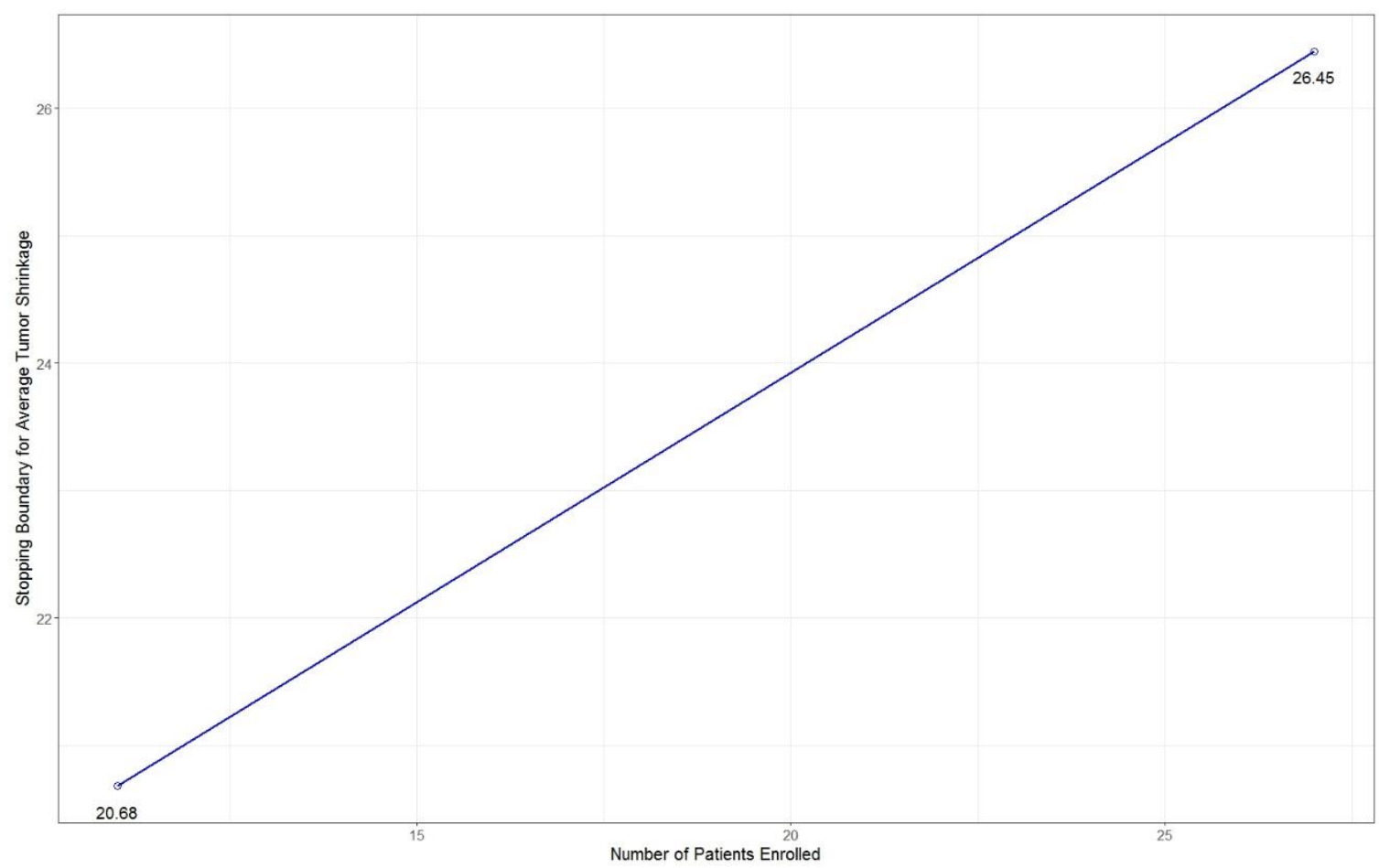

\$'Minimax Design`

[1]

Total Sample Size $\quad 26$

Sample Size for the First Stage 14

Futility Boundaries at the End of the First Stage $\quad 21.048$

Futility Boundaries at the End of the Second Stage 26.575

Probability of Early Termination $\quad 0.578$

Expected Sample Size at null $\quad 19.067$

Type I Error $\quad 0.045$

Type II Error $\quad 0.200$

pT $\quad 0.950$

$\mathrm{pL} \quad 0.060$

[2] 


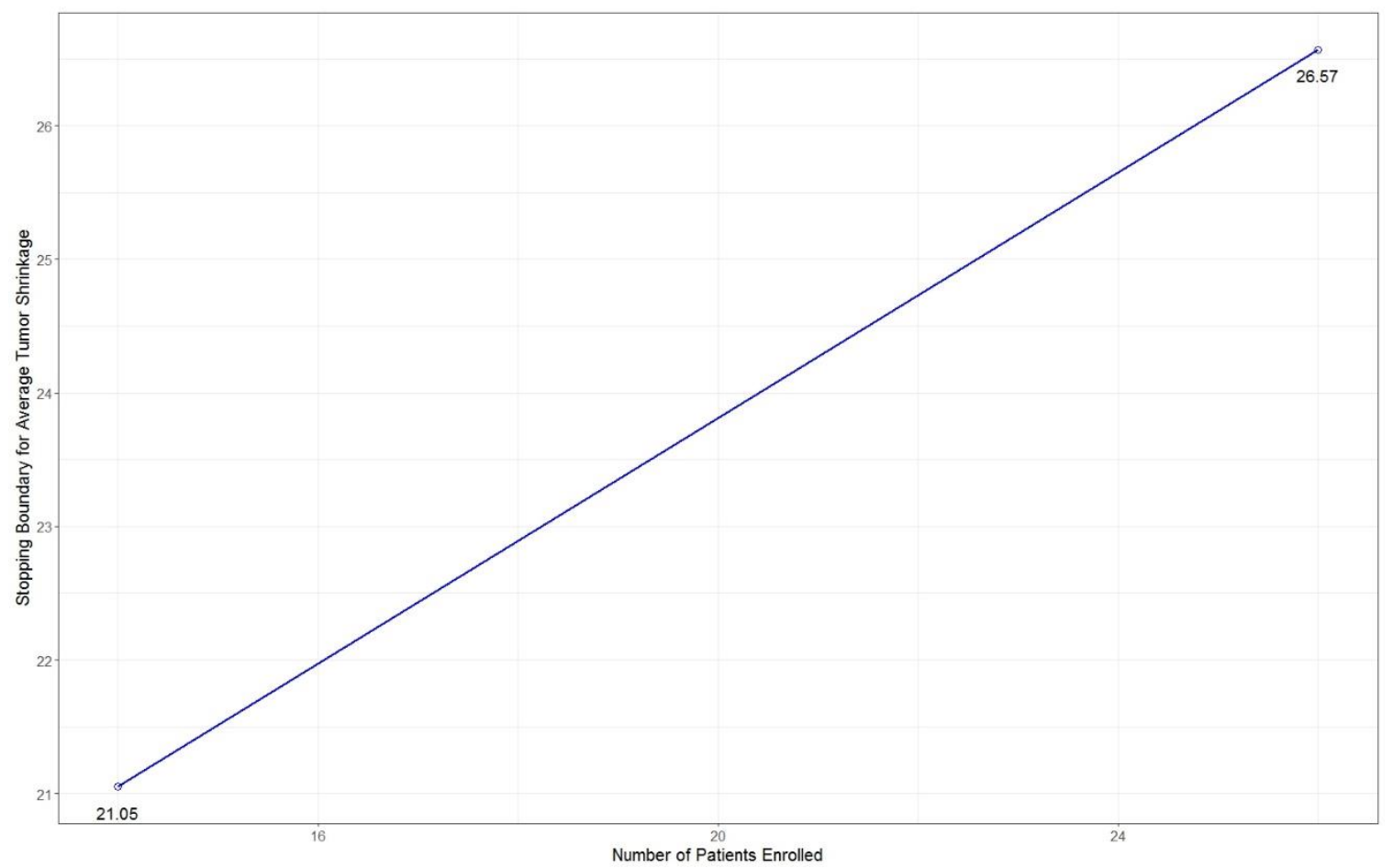

\section{Example 2:}

Although the current version of the software is not designed to search for the desired $\mathrm{pU}$ within a user-defined range, it has the capacity to allow for early stopping due to efficacy. The user can specify $\mathrm{pU}$, which is usually a large positive number between 0 and 1 , for example, above 0.9 . If the predictive probability is greater than $\mathrm{pU}$, say 0.95 , indicating that given the information from the current data, we will have $95 \%$ probability to conclude that the treatment is efficacious by the end of the study, if data had the same trend. Therefore, in the same scenario for example 1, if we designed the trial which to allow for early stopping either due to futility or superiority, the R code and the output are as follows: 
Example $2<-$ par2stage $($ sigma $=20$, tau $=20$, theta $=20, \mathrm{mu} 0=20, \mathrm{mu} 1=30$, pTlow $=0.9$, pThigh $=0.99$, pTby $=0.01$, pLlow $=0.01$, pLhigh $=0.1, \mathrm{pLby}=0.01, \mathrm{pU}=$ 0.95 , pre.typeIerror $=0.05$, pre typeIIerror $=0.2$, plot_yes $=\mathrm{T}$ )

\$Optimal Design`

[1]

Total Sample Size $\quad 27$

Sample Size for the First Stage $\quad 10$

Futility Boundaries at the End of the First Stage $\quad 20.215$

Futility Boundaries at the End of the Second Stage $\quad 26.447$

Efficacy Boundaries at the end of the First Stage $\quad 35.340$

Efficacy Boundaries at the End of the Second Stage $\quad 26.447$

Probability of Early Termination $\quad 0.521$

Expected Sample Size at null $\quad 18.140$

Type I Error $\quad 0.048$

Type II Error $\quad 0.197$

pT $\quad 0.950$

pL $\quad 0.100$

[2] 


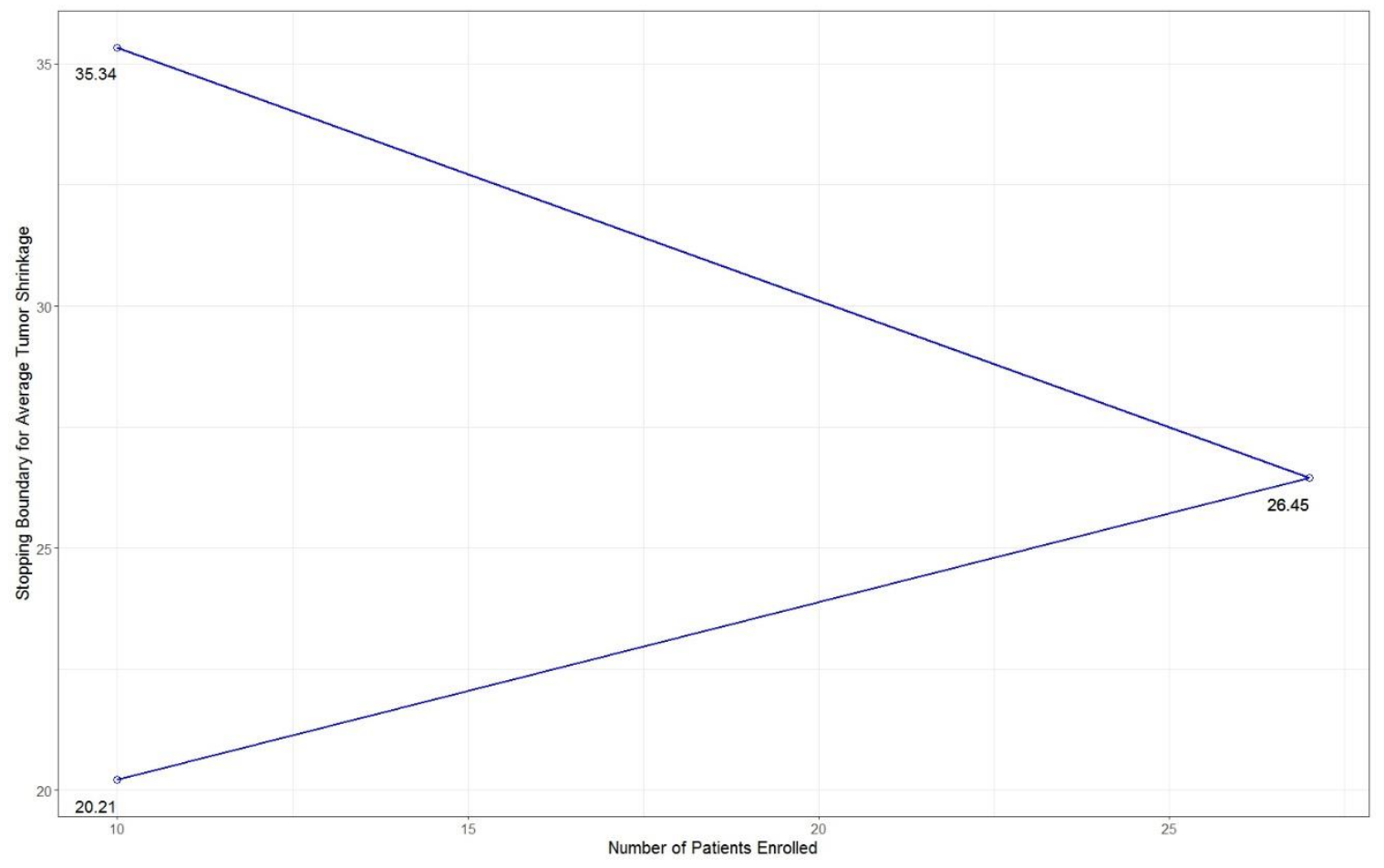

\$'Minimax Design`

[1]

Total Sample Size $\quad 26$

Sample Size for the First Stage 13

Futility Boundaries at the End of the First Stage 20.923

Futility Boundaries at the End of the Second Stage $\quad 26.575$

Efficacy Boundaries at the end of the First Stage $\quad 33.388$

Efficacy Boundaries at the End of the Second Stage $\quad 26.575$

Probability of Early Termination $\quad 0.574$

Expected Sample Size at null $\quad 18.538$

Type I Error $\quad 0.048$

Type II Error $\quad 0.199$

pT $\quad 0.950$

$\mathrm{pL} \quad 0.070$

[2] 


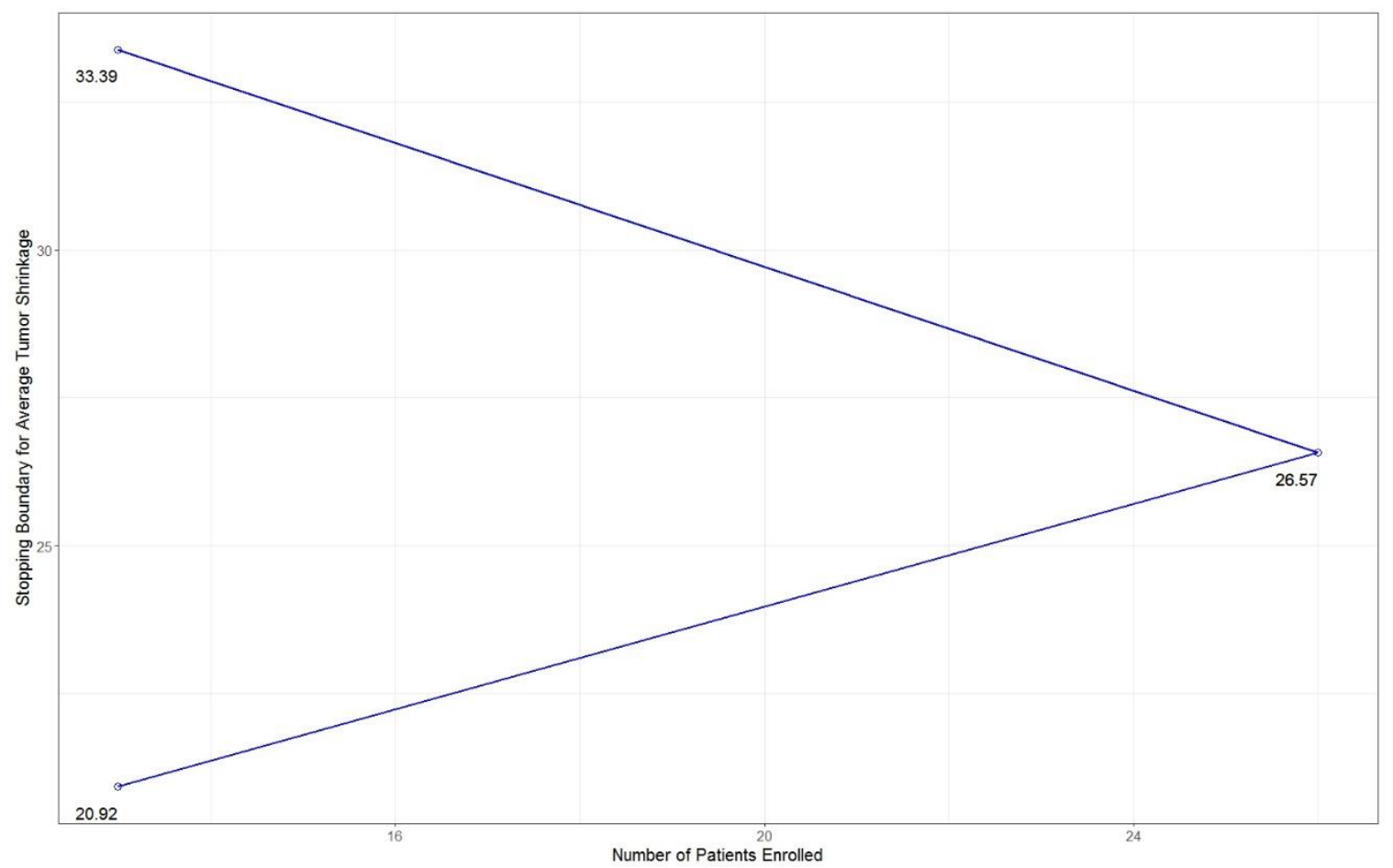

The design operating characteristics are very similar to the design that only allows for early stopping due to futility. In the output, there are two more operating characteristics "Efficacy Boundaries at the end of First Stage" and "Efficacy Boundaries at the end of Second Stage". The interpretation here is: in terms of the optimal design, after enrolling 10 patients, if the average tumor shrinkage is less than $20.215 \%$, we should stop the trial early for futility; if the average tumor shrinkage is greater than $35.340 \%$, we should stop the trial early for efficacy; otherwise we should continue to the second stage and enroll 10 additional patients. Both optimal designs (Example 1 and Example 2) yield a total sample size of 27 , and both minimax designs yield a total sample size of 26. pL and pT were also very similar for both optimal and minimax designs.

According to our simulation experience, the operating characteristics arising from the design allowing for early stopping due to efficacy will get close to those from the futility design when $\mathrm{pU}$ gets close to 1 . When $\mathrm{pU}$ is smaller, say 0.9 , the sample size required for 
the first stage will be larger. The probability of early termination also increases. The total sample size will be 26 with the probability of early termination 0.722 .

Example 1 and 2 explain the usage of function par2stage to perform the two-stage design. Both optimal design and minimax design are included in the output. The stopping boundaries are also displayed if the value for "plot_yes" argument is "T".

\subsubsection{Multi-stage design for early stopping due to both futility and efficacy: use of parmultistage}

\section{Example 3:}

Consider the same scenario from the previous examples in the two-stage design setting. The investigators want to incorporate more than one interim look during the entire trial. The standard therapy or historical control will lead to tumor shrinkage up to $20 \%$, and the clinical investigators want to test if the new therapy will improve the tumor shrinkage up to at least $30 \%$. All the other parameters are assumed to be the same.

In current version, for the multistage design, we are only allowed to search the range for $\mathrm{pT}$, but not for $\mathrm{pL}$ and $\mathrm{pU}$, due to intensive computation time. The user should pre-specify a single value for $\mathrm{pL}$ and $\mathrm{pU}$, for example, $\mathrm{pL}=0.1$ and $\mathrm{pU}=0.95$. Usually, as mentioned above, $\mathrm{pL}$ is a small number and $\mathrm{pU}$ is a large number between 0 and 1 . The pre-specified type I error rate and type II error rate were still set to be 0.05 and 0.2 . In addition, this current version can only display the minimax design also due to the computing time and searching strategy. If the investigators want to incorporate 3 interim looks during the entire trial with sample size equally spaced and the searching range for $\mathrm{pT}$ is still set as $[0.9,0.99]$, the R-code to execute is as follows:

Example3 <- parmultistage (continuous $=\mathrm{F}$, first.look $=$ NULL, num.interim $=3$, equal. spaced $=\mathrm{T}$, user.p $=\mathrm{NULL}$, sigma $=20$, tau $=20, \mathrm{mu} 0=20, \mathrm{mu} 1=30$, theta $=20$, 
pTlow $=0.9$, pThigh $=0.99$, pTby $=0.01, \mathrm{pL}=0.1, \mathrm{pU}=0.95$, pre.typeIerror $=0.05$, pre.typeIIerror $=0.2$, plot_yes $=\mathrm{T}$ )

$[[1]]$

\section{Minimax Design}

Cumulative sample size for each interim look $\quad \begin{array}{llll}7 & 14 & 21 & 29\end{array}$

$\begin{array}{lllll}\text { Futility Boundaries } & 18.00 & 21.42 & 23.34 & 26.21\end{array}$

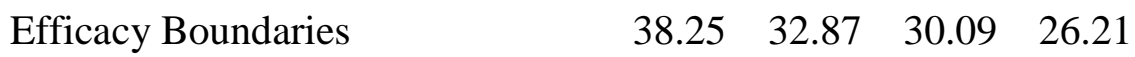

Probability of Early Termination $\quad 0.84$

Expected Sample Size under H0 24.67

Type I Error $\quad 0.05$

Type II Error $\quad 0.19$

pT 0.95

[[2]]

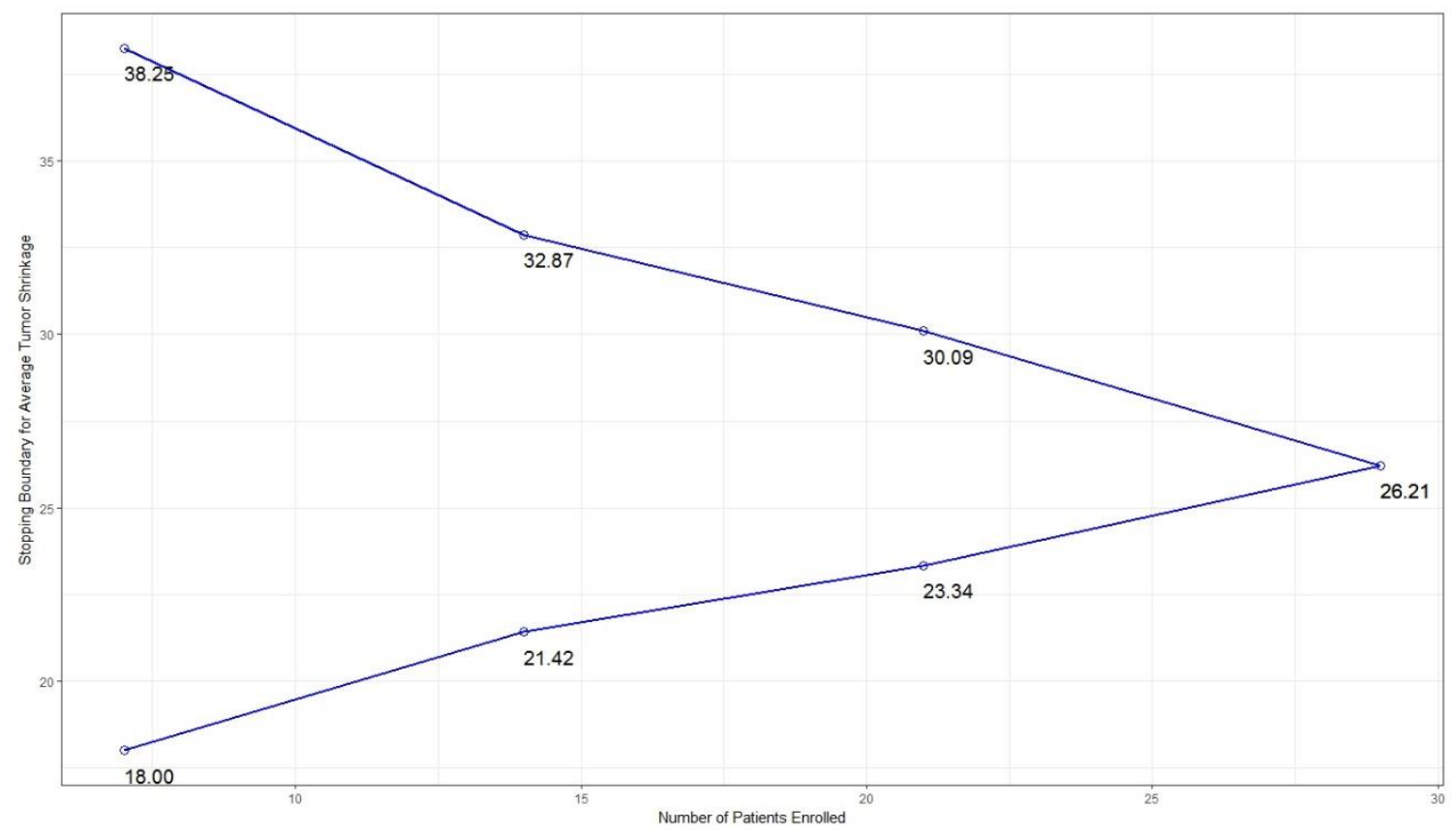

In the $\mathrm{R}$ arguments, just to set the num.interim equal to 3 , which is the number of interim looks desired during the trial, and to set equal.spaced $=\mathrm{T}$, indicating the same 
number of patients at each stage is desired. Sometimes, when we set the interim looks equally spaced through the trial, sample size in the last stage may deviate slightly from previous stages. In this example, the sample size for the last stage is 8 , instead of 7 as the first three stages. This discrepancy is due to rounding errors. If we set plot_yes $=\mathrm{T}$, the output from parmultistage function is a list containing two elements. The first element is the operating characteristics for the minimax design resulting from the user-specified parameters, including the sample size and futility/ efficacy boundaries for each interim look, probability of termination, expected sample size under the null hypothesis and type I/II error rates. The second element is a plot for stopping boundaries, with y-axis being the upper and lower boundaries for trial stopping rules, and $\mathrm{x}$-axis being the cumulative number of patients at each interim look. To hide the plot, set plot_yes equal to F.

\section{Example 4:}

If the investigators prefer not to space looks at the data equally, they can also define the cumulative proportion of the sample size regarding each interim look. For example, instead of equally spaced interim looks, the investigators can set user.p $=\mathrm{c}(0.1,0.4,0.8$, 1.0) and equal.spaced $=\mathrm{F}$, if they want to analyze the data from the trial after $10 \%, 40 \%$, $80 \%$ and $100 \%$ of the patients are enrolled. They can stop the trial at each of the interim looks either due to futility or efficacy. The R code is as follows:

Example4 <- parmultistage $($ continuous $=\mathrm{F}$, first.look $=$ NULL, num.interim $=3$, equal. spaced $=F$, user.p $=c(0.1,0.4,0.8,1.0)$, sigma $=20$, tau $=20$, mu0 $=20$, mu $1=30$, theta $=20, \mathrm{pTlow}=0.9, \mathrm{pThigh}=0.99, \mathrm{pTby}=0.01, \mathrm{pL}=0.1, \mathrm{pU}=0.95$, pre.typeIerror $=0.05$, pre.typeIIerror $=0.2$, plot_yes $=\mathrm{T}$ ) 
The output is as follows:

[[1]]

Minimax Design

Cumulative sample size for each interim look

$\begin{array}{llll}4 & 13 & 26 & 32\end{array}$

Futility Boundaries

$\begin{array}{llll}14.42 & 20.97 & 24.15 & 26.29\end{array}$

Efficacy Boundaries

$\begin{array}{llll}44.56 & 33.75 & 29.13 & 26.29\end{array}$

Probability of Early Termination $\quad 0.9$

Expected Sample Size under H0 24.9

Type I Error

0.04

Type II Error

0.2

pT

0.96

[[2]]

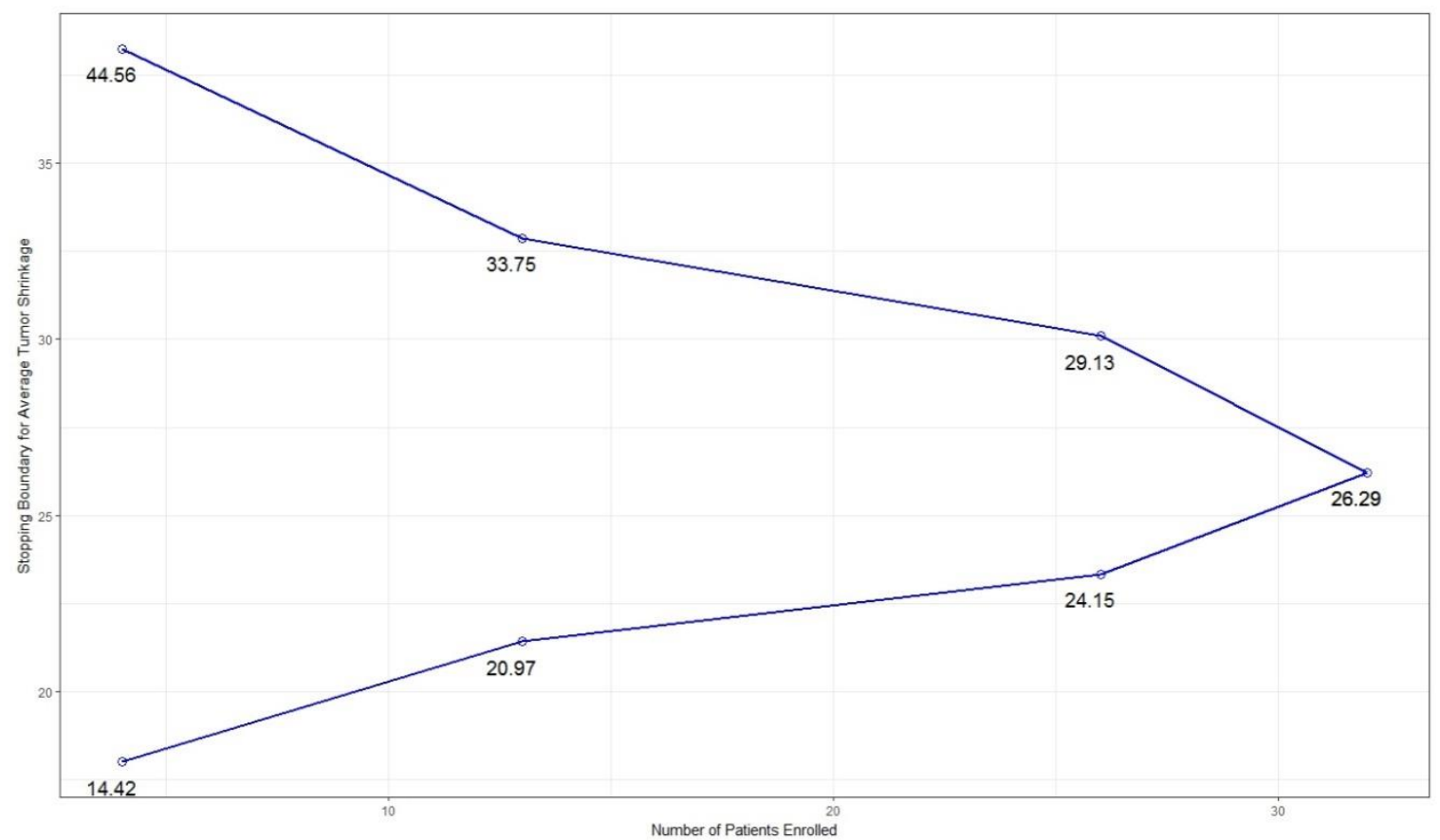

The results are different from the design with equally spaced interim looks. The total sample size required is larger than the design with equally spaced interim looks. Given different sample sizes at each interim look, as well as different $P_{T}$ yielding the minimax design, the futility and efficacy boundaries are also different between two designs. 


\section{Example 5:}

If the investigators want to continuously monitor the trial, especially when the effect size is large, they can specify the argument "continuous $=\mathrm{T}$ ". In addition, they can specify the proportion of the total sample size they plan for the first interim look. For example, when the effect size is 1 , that is sigma $=$ tau $=10$, and all the other parameters the same as previous examples, the investigators want to monitor the trial continuously after $50 \%$ of the patients are enrolled. In this scenario, we can specify the arguments of "continuous $=\mathrm{T}$ " and "first.look $=0.5 "$. The $\mathrm{R}$ code necessary to run this scenario is

Example5 <- parmultistage $($ continuous $=\mathrm{T}$, first.look $=0.5$, num.interim $=$ NULL , equal. spaced $=F$, user.p $=$ NULL, sigma $=10$, tau $=10$, mu0 $=20$, mul $=30$, theta $=20$, pTlow $=0.9$, pThigh $=0.99$, pTby $=0.01, \mathrm{pL}=0.1, \mathrm{pU}=0.95$, pre.typeIerror $=0.05$, pre.typeIIerror $=0.2$, plot_yes $=\mathrm{T}$ )

The output is as follows:

$[[1]]$

\section{Minimax Design}

Cumulative sample size for each interim look

$\begin{array}{llll}4 & 5 & 6 & 7\end{array}$

Futility Boundaries

$22.48 \quad 23.46 \quad 24.42 \quad 26.28$

Efficacy Boundaries

$\begin{array}{llll}32.50 & 30.63 & 28.98 & 26.28\end{array}$

Probability of Early Termination $\quad 0.9$

Expected Sample Size under H0 $\quad 6.70$

Type I Error $\quad 0.05$

Type II Error $\quad 0.19$

pT 0.94

[[2]] 


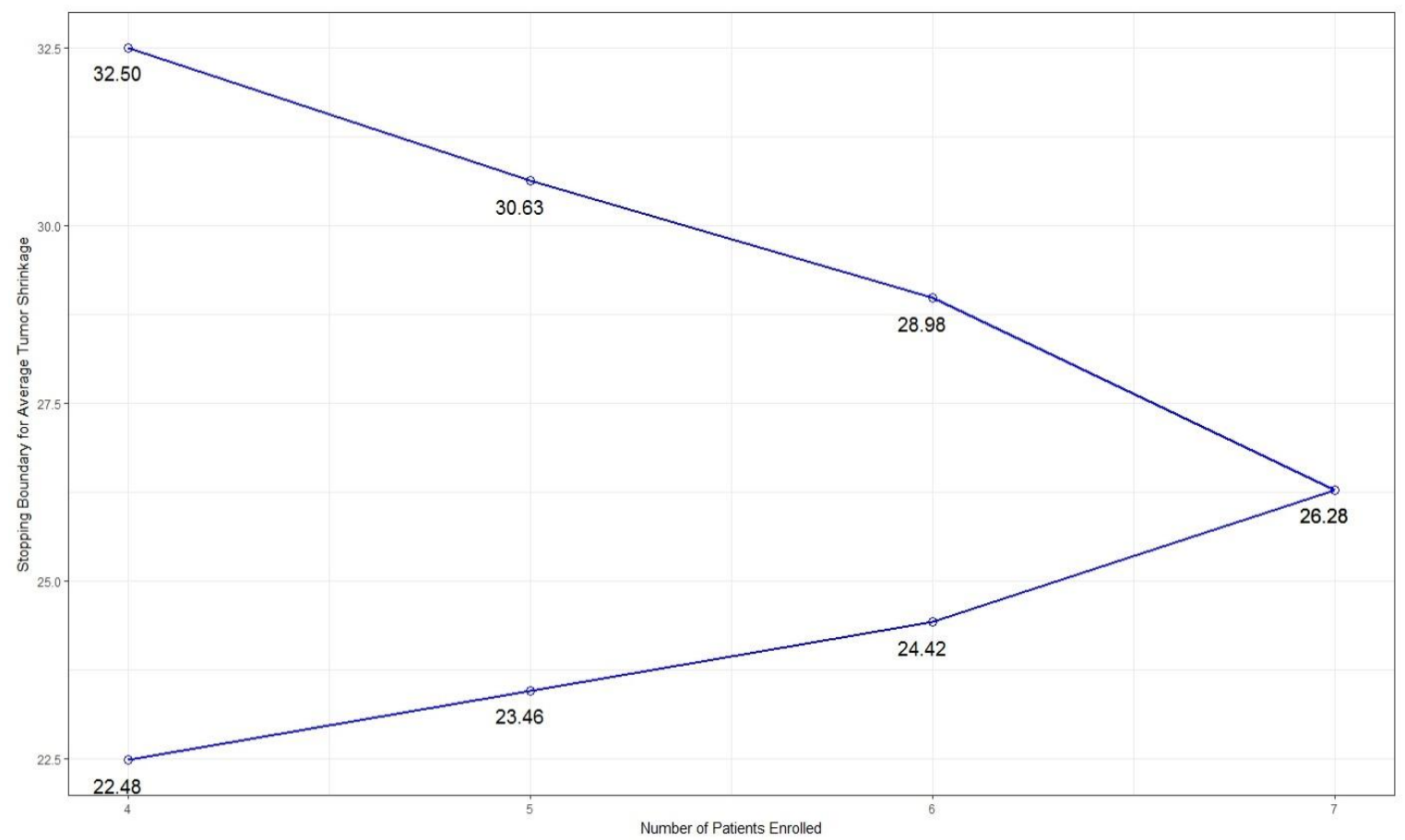

\subsection{Discussion}

In this paper, we detailed the usage and implementation of the package PPSC corresponding to our single-arm predictive probability two- and multi- stage design for continuous outcomes. Package PPSC is an R package that provides a grid search for desired optimal and minimax design for continuous normal outcome based on predictive probability. Both optimal and minimax designs satisfy pre-specified type I and type II error rates. Package PPSC can be utilized to monitor the trial and stop the trial early either due to futility or efficacy. The function to conduct the two-stage trial is originally designed for futility trial, while it has the capacity to accommodate for early stop due to superiority. The function to conduct the multi-stage design can accommodate the situations with equal sample size at each stage, unequal sample size at each stage, and continuous monitoring of the trial. 
On the other hand, there are several limitations to the current version of Package PPSC. First, Package PPSC is currently available only for normally distributed outcomes with conjugated priors. Second, in terms of multi-stage design, especially for the purpose of continuous monitoring, this version can only incorporate up to 40 stages due to the current package R2Cuba for multidimensional numerical integration [77]. Third, for twostage design, the package PPSC yields both optimal design and minimax design in the output. A user-defined range for $P_{T}$ and $P_{L}$ is searched to find both optimal and minimax designs. However, only minimax design is shown for multi-stage setting, due to the grid searching strategy and algorithm we employed. In the multi-stage setting, we restrict $P_{L}$ and $P_{U}$ as a user-defined value and search for a user-defined range for $P_{T}$ to find the minimax design.

In summary, we extended Lee and Liu's predictive probability approach into twostage and multi-stage setting for continuous endpoints. In practice, these two designs are more relevant for treatment effects with short observation time. In the scenario where the treatment effect will take a long period, such as several weeks or months, to observe, this design is not quite useful. That is when the long-time treatment effect is expected in practice, patients will have been enrolled in later stages in the trial, since the patients will generally recruited continuously. Of course, it also depends on the institutes and recourses to enroll patients. We also developed an R package PPSC to implement the described designs. This package will be submitted to CRAN shortly after completion of this study and will then be freely accessible in R. All necessary functions are available upon request. 
Table 4.1 Input options for function par2stage

\begin{tabular}{|c|c|}
\hline Name & Role \\
\hline sigma & $\begin{array}{l}\text { The standard deviation from the user pre-specified normal likelihood } \\
\text { function. }\end{array}$ \\
\hline tau & $\begin{array}{l}\text { The standard deviation from the user pre-specified normal prior } \\
\text { distribution. }\end{array}$ \\
\hline theta & The mean from the user pre-specified normal prior distribution. \\
\hline mu0 & The maximum uninteresting mean from the null hypothesis. \\
\hline mu1 & The minimum interesting mean from the alternative hypothesis. \\
\hline pTlow & $\begin{array}{l}\text { The lower boundary for searching range of } P_{T} . P_{T} \text { is a value above } 0.8 \text {. } \\
\text { If the searching range for } P_{T} \text { is from } 0.9 \text { to } 0.99 \text {, then pTlow }=0.9 \text {. }\end{array}$ \\
\hline pThigh & $\begin{array}{l}\text { The higher boundary for searching range of } P_{T} . P_{T} \text { is a value above } 0.8 \text {. } \\
\text { If the searching range for } P_{T} \text { is from } 0.9 \text { to } 0.99 \text {, then pThigh }=0.99 \text {. }\end{array}$ \\
\hline pTby & The searching increment for $P_{T}$. \\
\hline pLlow & $\begin{array}{l}\text { The lower boundary for searching range of } P_{L} . P_{L} \text { is usually a small } \\
\text { value below } 0.1 \text {. If the searching range for } P_{L} \text { is from } 0.01 \text { to } 0.1 \text {, then } \\
\text { pLlow }=0.01 \text {. }\end{array}$ \\
\hline pLhigh & $\begin{array}{l}\text { The higher boundary for searching range of } P_{L} . P_{L} \text { is usually a small } \\
\text { value below } 0.1 \text {. If the searching range for } P_{L} \text { is from } 0.01 \text { to } 0.1 \text {, then } \\
\text { pLhigh }=0.1 \text {. }\end{array}$ \\
\hline pLby & The searching increment for $P_{L}$. \\
\hline $\mathrm{pU}$ & $\begin{array}{l}\text { The efficacy boundary for predictive probability, which is usually a } \\
\text { value above } 0.9 \text {. }\end{array}$ \\
\hline pre.typeIerror & The user pre-specified nominal type I error rate constraint. \\
\hline pre.typeIIerror & The user pre-specified nominal type II error rate constraint. \\
\hline plot_yes & The option to choose if displaying the stopping boundary plot. \\
\hline
\end{tabular}


Table 4.2 Input options for function parmultistage

\begin{tabular}{|c|c|}
\hline Name & Role \\
\hline continuous & $\begin{array}{l}\text { The option to choose continuous monitoring or not. The default value } \\
\text { is F. }\end{array}$ \\
\hline first.look & $\begin{array}{l}\text { The proportion of the total sample for the first interim look. The default } \\
\text { value is NULL. The user should specify a number when continuously } \\
\text { monitoring is desired. }\end{array}$ \\
\hline num.interim & The number of interim looks during the trial. \\
\hline equal.space & The option to set equally spaced interim looks. The default value is $\mathrm{T}$. \\
\hline user.p & The user defined proportion of sample size for each interim looks. \\
\hline sigma & $\begin{array}{l}\text { The standard deviation from the user pre-specified normal likelihood } \\
\text { function. }\end{array}$ \\
\hline tau & $\begin{array}{l}\text { The standard deviation from the user pre-specified normal prior } \\
\text { distribution. }\end{array}$ \\
\hline theta & The mean from the user pre-specified normal prior distribution. \\
\hline mu0 & The maximum uninteresting mean from the null hypothesis. \\
\hline mu1 & The minimum interesting mean from the alternative hypothesis. \\
\hline pTlow & $\begin{array}{l}\text { The lower boundary for searching range of } P_{T} . P_{T} \text { is a value above } 0.8 \text {. } \\
\text { If the searching range for } P_{T} \text { is from } 0.9 \text { to } 0.99 \text {, then pTlow }=0.9 \text {. }\end{array}$ \\
\hline pThigh & $\begin{array}{l}\text { The higher boundary for searching range of } P_{T} . P_{T} \text { is a value above } 0.8 \text {. } \\
\text { If the searching range for } P_{T} \text { is from } 0.9 \text { to } 0.99 \text {, then pThigh }=0.99 \text {. }\end{array}$ \\
\hline pTby & The searching increment for $P_{T}$. \\
\hline $\mathrm{pL}$ & $\begin{array}{l}\text { The user pre-specified value for } P_{L} \text {, which is usually a small value } \\
\text { below } 0.1 \text {. }\end{array}$ \\
\hline $\mathrm{pU}$ & $\begin{array}{l}\text { The user pre-specified value for } P_{U} \text {, which is usually a large value } \\
\text { above } 0.9 \text {. }\end{array}$ \\
\hline pre.typeIerror & The user pre-specified nominal type I error rate constraint. \\
\hline pre.typeIIerror & The user pre-specified nominal type II error rate constraint. \\
\hline plot_yes & The option to choose if displaying the stopping boundary plot. \\
\hline
\end{tabular}




\section{CHAPTER FIVE}

\section{Summary and Conclusion}

\subsection{Conclusion}

Phase II trials aim to identify promising agents or therapies to move forward to confirmative randomized phase III trials. In practice, RECIST criteria is commonly applied to categorize the tumor shrinkage into complete response, partial response, progressive disease and stable disease [1]. Response rate, which is the combination of complete and partial response, with at least a $30 \%$ decrease in the sum of the longest diameter of the target lesions, is usually used as a secondary endpoint in most of the cancer trials. However, as shown in the previous studies, this dichotomization not only leads to loss of statistical efficiency, but greatly increases the required sample size [5, 54-56, 58].

Interim strategies are of great importance in each stage of oncology clinical trials, and at least one interim look is usually encouraged when designing the trial. The purpose of interim looks is to terminate the trial early for safety, futility, or efficacy, and to facilitate the trial process and protect patients' clinical benefits [14]. Two-stage design is widely used in phase II trials, one of which is Simon's two-stage design in a single-arm setting for binary outcomes, such as response rate [19]. It has been extended in many different ways and has been summarized in the introduction part. Regarding multi-stage designs, the group-sequential approach is usually used in practice. A groupsequential design for clinical trial is usually to analyze the interim data after groups of patients are enrolled. In this scheme, the number of patients at each stage is pre-specified and the trial continues as long as the underlying test statistics keeps within a specified boundary. In the randomized setting, group sequential designs are well developed and 
widely applied in practice to identify and select new therapies [62, 63, 74]. For single-arm settings, in particular, Chang et al. proposed a group-sequential design for binary outcomes with minimized average expected sample size under the null and alternative hypotheses in phase II trials under the frequentist framework [80]. When compared with frequentist designs, Bayesian designs are more flexible. Lee and Liu proposed a predictive probability design, on which we developed our researches [37].

In this dissertation, we proposed a two-stage design and a multi-stage design for continuous endpoints based on predictive probability and developed an $\mathrm{R}$ package for these two designs. The two-stage design is mainly focused on futility early termination while it has the capacity to stop for efficacy. The multi-stage design has the flexibility to allow early stopping either due to futility or efficacy. Both designs are flexible and user-friendly. The users can choose to monitor the trial continuously, or to monitor the trial with prespecified number of stages, in which the users have the options to set equal or unequal proportions of patients at each stage. Both designs meet pre-specified type I and type II error rate constraints.

For both design options, we obtained similar decreased sample size as in previous studies, when using tumor shrinkage instead of response rate, as continuous variable and maintained the frequentist operating characteristics at the same time $[5,56]$. We also explored and evaluated the roles of priors on the effect of the design operating characteristics. The results were shown to be fairly robust, although the comparison was illustrative but not conclusive. Assessed priors yielded similar sample sizes and other operating characteristics were slightly different. We also compared our results with designs based on posterior designs, and the results were very similar. However, when compared 
with posterior probability design, this predictive probability approach has the advantages that it mimics the decision making process in reality and more intuitive. It answers the question that if additional data are collected, is the trial likely to show convincing evidence against the null hypothesis and in favor of the alternative hypothesis. The predictive probabilities approach is able to model the endpoint using earlier information that is informative about the final outcome.

\subsection{Strength and Limitations}

\section{Strengths:}

In general, multi-stage is usually more efficient than two-stage or three-stage design. It is more efficient to stop the trial early for futility or superiority, which will save resources, protect patients, and accelerate the process of drug development. When compared with designs using binary outcomes, using continuous endpoints utilize more information from the data and, therefore, reduce the sample size. Both of our designs based on predictive probability, while maintaining the frequentist operating characteristics.

One of the major strengths for this study is the flexibility to implement and making it an appealing alternative to other designs. In terms of two-stage design, the users have the option to stop the trial early only due to futility, or to stop the trial early due to efficacy with a specific value of $P_{T}$. Regarding the multi-stage design, it allows continuous monitoring up to 40 stages. It is particularly useful when the effect size is large, and the investigators want to monitor the trial continuously. Further, this multi-stage design allows the flexibility of setting either equal or unequal numbers of patients at each stage when the effect size is moderate or small. To choose the design with equal numbers of patients at 
each stage, the users just need to specify the number of interim looks they prefer. On the other side, if the users would like designs with unequal sample size for each stages, they can specify the cumulative proportion of the total sample size at each interim look.

Another strength for this current study is that although the trial stopping boundaries are obtained from the predictive probability given the information from current data, which resembles more closely to the decision making process of conducting clinical trials in practice, it relies on parameters $P_{L}, P_{U}$ and $P_{T}$ to control the type I and type II error rate constraints in the meanwhile. With this hybrid setting, our designs were shown to possess the desirable operating characteristics and maintain both type I and type II error rates.

Further, it has been shown that our design gave fairly robust results across different priors. The choice of priors is important in Bayesian clinical trial designs. We compared the results across "weak/strong" and "optimistic/ pessimistic" priors and all the results were very similar across different prior combinations, although more thorough assessment of priors' effect, especially those non-conjugated priors, should be conducted in the future.

Another strength is that we develop an R package PPSC to implement both twostage and multi-stage design. It is important to have a user-friendly tool available, which makes the two designs accessible in the future for the collaboration between biostatisticians and investigators.

\section{Limitations:}

There are several limitations in current two-stage and multi-stage designs, which we plan to address in the future. Firstly, for both designs, we assume the standard deviation $\sigma$ for the likelihood function of the studying continuous endpoint is known, in line with previous studies. It is reasonable, since in practice this value could be obtained from 
precious trials or preclinical studies. However, when there is little prior information is available, especially for those novel drugs or biomarkers, it is not a valid assumption. Thus, unknown variance may be explored in the future. Secondly, prior are important in Bayesian clinical trial designs. It is always challenging and controversial to choose a suitable prior on the parameter of interest. For both designs, we only assessed the conjugate priors for the mean, assuming variance is known, and explored a little bit on the roles of pessimistic and optimistic priors. There could be clinical situations where the prior does not follow a normal distribution and thus the resulting posterior does not have a closed form. More exploration is needed in this regard. We plan to look at other non-normal priors, such as log-normal distributions in the future.

In terms of the multistage design, although our R package has the flexibility for user-defined sample size for each interim look, we need to set the value of $P_{L}$ and $P_{U}$ and only search for $P_{T}$ to find the desired design. In addition, we need to do a thorough evaluation on how to space the interim looks to achieve the most desirable design and how it will affect the design properties. Further, our grid searching strategy can only accommodate minimax design due to computation time due to the grid search strategy we employed. It will be interesting to see how the probability of early termination under the null hypothesis and compare the expected sample size from the optimal design with the smallest sample size desired.

\subsection{Future Directions}

We plan to extend this current study in different directions. There are several future

directions in common for both two-stage design and multi-stage designs. First, since 
current design can only operate on conjugator priors based on normal likelihood, we would like to extend both current two-stage design and multi-stage design to accommodate nonconjugate priors. To do this, we will utilize the WinBUGS software and the R package R2WinBUGS to handle non-conjugate priors [81, 82]. Further, we only explored situations where the continuous outcome follows a normal distribution. However, as mentioned above, it may not be realistic in practice. Thus, we plan to explore other distributions for the continuous endpoints. Second, in practice, the likelihood variance may not be known in advance, we would like to incorporate the likelihood variance as an unknown parameter into the design. Third, the current design is best for the short-term endpoints, which can be evaluated without affecting the continuous process of patients' recruitment. However, this design may not be appropriate for those endpoints that need longer time to be obtained and evaluated. Recently, with the development of immunotherapy in oncology, there are several proposed designs taking into the treatment lagged time effect for the survival endpoints [83-85]. One future direction is to incorporate time factor or delayed treatment effect into the design for the continuous endpoints. Another possible future direction is evaluate this continuous endpoint and survival outcome simultaneously, which may be more interesting in terms of clinical impact.

Lastly, we also plan to optimize and update the searching strategy in the future. For the multi-stage design described in chapter 3, the current version only allows searching for a range of $P_{T}$ for the minimax design. We would like to optimize the searching strategy to allow $P_{L}$ and $P_{U}$ search as well as the optimal design output. In addition, due to the current algorithm, we can only accommodate up to 40 stages during the trial due to the dependency 
of the cubature package [77]. We would like to expand it into continuous monitoring without limitation.

In conclusion, we developed two predictive probability interim designs for continuous endpoints and an $\mathrm{R}$ package to implement in this dissertation. The two designs are flexible and well preserve statistical properties. It provides an alternative option for continuous endpoints when a single-arm design with flexible interim stopping is desired. As research rapidly moves to incorporate more immunotherapies and targeted therapies, these designs will accommodate new types of outcomes while allowing for flexible stopping rules for futility and/or efficacy to continue optimizing trial resources and prioritize agents with compelling early phase data. 


\section{APPENDICES}

\section{A1 Transformations from binary endpoints $(p)$ to continuous endpoints}

$(\mu)[5]$ (From Wason et al., 2011).

Let $p_{0}$ denote the response rate under the null hypothesis and $p_{1}$ denote

the response rate under the alternative hypothesis. $\mu_{0}$ is the tumor shrinkage under the null hypothesis and $\mu_{1}$ is under the alternative hypothesis. $\sigma$ is the standard deviation of the continuous endpoint, which is assumed to be known. $d$ is the cut-off the ratio of dichotomization, which is $30 \%$ with RECIST criteria [1]. $X$ is denoted as the random variable for the continuous endpoint, such as tumor shrinkage. $Z$ is the standard normal random variable.

$$
p_{0}=\operatorname{Pr}(X>d)=\operatorname{Pr}\left(\sigma Z+\mu_{0}>d\right)=1-\Phi\left(\frac{d-\mu_{0}}{\sigma}\right)
$$

So that:

$$
\mu_{0}=d-\sigma \Phi^{-1}\left(1-p_{0}\right)
$$

Similarly,

$$
\mu_{1}=d-\sigma \Phi^{-1}\left(1-p_{1}\right)
$$




\section{A2 Frequentist operating characteristics calculation for two-stage design}

\section{to terminate early for efficacy.}

If the investigators prefer to terminating the trial early for both futility and efficacy, we can rewrite the $\varphi(\cdot)$ in chapter one in the form of rejecting the null hypothesis at each stage. We denote it as $\varphi^{*}(\cdot)$. Thus,

$$
\varphi^{*}\left(\mu_{0}, \sigma, n_{1}, n_{2}, \bar{X}_{n_{1}}, \bar{X}_{L}, \bar{X}_{U}, \bar{X}_{L}^{\prime}\right)=P_{\mu}\left(\bar{X}_{n_{1}} \geq \bar{X}_{U}\right)+\int_{\bar{X}_{L}}^{\bar{X}_{U}} f_{\mu}(x) P_{\mu}\left(\bar{X}_{n_{1}+n_{2}} \geq \bar{X}_{U}^{\prime}\right) d x
$$

Similar as $\varphi($.$) , the first part of \varphi^{*}($.$) is the probability of rejecting the null hypothesis at$ the first stage and the second part if the probability of rejecting the null at the end of second stage.

The corresponding type I and type II error rates can be written as:

$$
\begin{gathered}
\alpha=\varphi^{*}\left(\mu_{0}, \sigma, n_{1}, n_{2}, \bar{X}_{n_{1}}, \bar{X}_{L}, \bar{X}_{U}, \bar{X}_{L}^{\prime}\right) \\
\beta=1-\varphi^{*}\left(\mu_{1}, \sigma, n_{1}, n_{2}, \bar{X}_{n_{1}}, \bar{X}_{L}, \bar{X}_{U}, \bar{X}_{L}^{\prime}\right)
\end{gathered}
$$

The probability of early termination under the null hypothesis is :

$$
\operatorname{PET}\left(\mu_{0}\right)=P_{\mu}\left(\bar{X}_{n_{1}} \leq \bar{X}_{L} \mid H_{0}\right)+P_{\mu}\left(\bar{X}_{n_{1}} \geq \bar{X}_{U} \mid H_{0}\right)
$$

And the expected sample size under the null hypothesis is the same as the situation for only stopping early for futility. 


\section{A3 Supplementary simulation results for two-stage designs.}

Table A3.1 Optimal two-stage design stopping early for futility

Table A3.2 Minimax two-stage design stopping early for futility

Table A3.3 Optimal two-stage design stopping early for efficacy

Table A3.4 Minimax two-stage design stopping early for efficacy

Table A3.5 Operating characteristics of optimal efficacy designs with different priors.

Table A3.6 Operating characteristics of minimax efficacy designs with different priors. 
Table A3.1 Operating characteristics of optimal two-stage design for continuous endpoints with early termination for futility. For both designs below, $P_{U}=1, \mu_{0}=20, \mu_{1}=30, \theta=\mu_{0}=20, \sigma=10,12.5,20$ and 50, $\tau=\sigma$. The first row for each effect size is with type I and type II error rate constraints $(\alpha, \beta)=(0.05,0.2)$ and the second row for each effect size is with $(\alpha, \beta)=(0.05,0.1)$.

\begin{tabular}{|c|c|c|c|c|c|c|c|c|c|c|c|}
\hline \multicolumn{12}{|c|}{ Optimal Design } \\
\hline Effect size & $\sigma$ & $P_{T}$ & $P_{L}$ & Nmax & $n_{1}$ & $\bar{X}_{L}$ & $\bar{X}_{L}^{\prime}$ & PET & En & Type I Error & Type II Error \\
\hline 1.0 & 10.0 & 0.945 & 0.100 & 7 & 3 & 21.49 & 26.46 & 0.60 & 4.59 & 0.040 & 0.199 \\
\hline & & 0.937 & 0.100 & 9 & 4 & 20.98 & 25.38 & 0.58 & 6.11 & 0.050 & 0.099 \\
\hline 0.9 & 11.1 & 0.936 & 0.094 & 8 & 3 & 20.25 & 26.34 & 0.52 & 5.42 & 0.049 & 0.200 \\
\hline & & 0.939 & 0.094 & 11 & 5 & 20.89 & 25.41 & 0.57 & 7.58 & 0.050 & 0.100 \\
\hline 0.8 & 12.5 & 0.938 & 0.100 & 10 & 5 & 21.67 & 26.38 & 0.62 & 6.91 & 0.050 & 0.199 \\
\hline & & 0.941 & 0.096 & 14 & 6 & 20.63 & 25.41 & 0.55 & 9.60 & 0.050 & 0.100 \\
\hline 0.7 & 14.3 & 0.941 & 0.085 & 13 & 6 & 20.85 & 26.43 & 0.56 & 9.09 & 0.050 & 0.200 \\
\hline & & 0.943 & 0.085 & 18 & 9 & 21.01 & 25.47 & 0.58 & 12.74 & 0.050 & 0.100 \\
\hline 0.6 & 16.7 & 0.942 & 0.100 & 18 & 7 & 20.30 & 26.34 & 0.52 & 12.29 & 0.050 & 0.199 \\
\hline & & 0.944 & 0.099 & 25 & 10 & 20.31 & 25.40 & 0.52 & 17.15 & 0.049 & 0.100 \\
\hline 0.5 & 20.0 & 0.945 & 0.100 & 26 & 11 & 20.69 & 26.39 & 0.55 & 17.81 & 0.048 & 0.199 \\
\hline & & 0.945 & 0.100 & 36 & 15 & 20.46 & 25.40 & 0.54 & 24.76 & 0.049 & 0.099 \\
\hline 0.4 & 25.0 & 0.945 & 0.098 & 40 & 17 & 20.57 & 26.40 & 0.54 & 27.65 & 0.050 & 0.200 \\
\hline & & 0.946 & 0.100 & 56 & 24 & 20.54 & 25.42 & 0.54 & 38.65 & 0.049 & 0.100 \\
\hline 0.3 & 33.3 & 0.946 & 0.099 & 71 & 31 & 20.68 & 26.40 & 0.55 & 49.49 & 0.050 & 0.200 \\
\hline & & 0.946 & 0.100 & 99 & 42 & 20.45 & 25.41 & 0.54 & 68.52 & 0.050 & 0.100 \\
\hline 0.2 & 50.0 & 0.947 & 0.100 & 160 & 71 & 20.77 & 26.41 & 0.55 & 110.93 & 0.049 & 0.200 \\
\hline & & 0.947 & 0.100 & 223 & 96 & 20.51 & 25.42 & 0.54 & 154.48 & 0.050 & 0.100 \\
\hline 0.1 & 100 & 0.947 & 0.100 & 638 & 279 & 20.66 & 26.41 & 0.51 & 442.83 & 0.050 & 0.200 \\
\hline & & 0.947 & 0.100 & 890 & 382 & 20.47 & 25.42 & 0.54 & 617.33 & 0.050 & 0.100 \\
\hline
\end{tabular}


Table A3.2 Operating characteristics of minimax two-stage design for continuous endpoints with early termination for futility. For both designs below, $P_{U}=1, \mu_{0}=20, \mu_{1}=30, \theta=\mu_{0}=20, \sigma=10,12.5,20$ and 50, $\tau=\sigma$. The first row for each effect size is with type I and type II error rate constraints $(\alpha, \beta)=(0.05,0.2)$ and the second row for each effect size is with $(\alpha, \beta)=(0.05,0.1)$.

\begin{tabular}{|c|c|c|c|c|c|c|c|c|c|c|c|}
\hline \multicolumn{12}{|c|}{ Minimax Design } \\
\hline Effect & $\sigma$ & $\mathrm{P}_{\mathrm{T}}$ & $\mathrm{P}_{\mathrm{L}}$ & N.max & $\mathrm{n} 1$ & $\overline{\bar{X}}_{L}$ & $\bar{X}_{L}^{\prime}$ & PET & En & Type I & Type II \\
\hline size & & & & & & & & & & Error & Error \\
\hline 1.0 & 10.0 & 0.945 & 0.100 & 7 & 3 & 21.49 & 26.46 & 0.60 & 4.59 & 0.040 & 0.199 \\
\hline & & 0.937 & 0.100 & 9 & 4 & 20.98 & 25.38 & 0.58 & 6.11 & 0.050 & 0.099 \\
\hline 0.9 & 11.1 & 0.947 & 0.031 & 8 & 1 & 6.11 & 26.74 & 0.11 & 7.26 & 0.042 & 0.189 \\
\hline & & 0.938 & 0.058 & 11 & 3 & 17.07 & 25.38 & 0.32 & 8.41 & 0.049 & 0.096 \\
\hline 0.8 & 12.5 & 0.938 & 0.100 & 10 & 5 & 21.67 & 26.38 & 0.62 & 6.91 & 0.050 & 0.199 \\
\hline & & 0.941 & 0.096 & 14 & 6 & 20.63 & 25.41 & 0.55 & 9.60 & 0.050 & 0.100 \\
\hline 0.7 & 14.3 & 0.945 & 0.044 & 13 & 7 & 20.53 & 26.57 & 0.54 & 9.77 & 0.047 & 0.200 \\
\hline & & 0.952 & 0.001 & 16 & 3 & 1.95 & 26.13 & 0.01 & 15.81 & 0.043 & 0.089 \\
\hline 0.6 & 16.7 & 0.941 & 0.063 & 18 & 6 & 18.04 & 26.31 & 0.39 & 13.36 & 0.050 & 0.188 \\
\hline & & 0.942 & 0.046 & 24 & 7 & 16.63 & 25.46 & 0.30 & 18.96 & 0.050 & 0.099 \\
\hline 0.5 & 20.0 & 0.945 & 0.080 & 25 & 18 & 23.09 & 26.52 & 0.74 & 19.79 & 0.050 & 0.200 \\
\hline & & 0.946 & 0.069 & 35 & 18 & 20.72 & 25.51 & 0.56 & 25.47 & 0.050 & 0.100 \\
\hline 0.4 & 25.0 & 0.940 & 0.055 & 39 & 14 & 17.85 & 26.30 & 0.37 & 29.66 & 0.050 & 0.189 \\
\hline & & 0.945 & 0.002 & 53 & 23 & 14.25 & 25.54 & 0.14 & 48.95 & 0.047 & 0.097 \\
\hline 0.3 & 33.3 & 0.941 & 0.069 & 69 & 24 & 18.23 & 26.32 & 0.40 & 51.12 & 0.050 & 0.194 \\
\hline & & 0.950 & 0.010 & 96 & 59 & 19.37 & 25.62 & 0.44 & 79.62 & 0.049 & 0.100 \\
\hline 0.2 & 50.0 & 0.949 & 0.080 & 155 & 149 & 25.46 & 26.59 & 0.91 & 149.55 & 0.050 & 0.200 \\
\hline & & 0.948 & 0.081 & 217 & 146 & 22.23 & 25.53 & 0.70 & 166.95 & 0.050 & 0.100 \\
\hline 0.1 & 100 & 0.943 & 0.006 & 591 & 282 & 15.69 & 26.51 & 0.23 & 518.48 & 0.049 & 0.199 \\
\hline & & 0.944 & 0.017 & 858 & 265 & 16.64 & 25.46 & 0.29 & 684.80 & 0.049 & 0.099 \\
\hline
\end{tabular}


Table A3.3 Operating characteristics of optimal two-stage design for continuous endpoints with early termination for efficacy. For both designs below, $P_{U}=0.95, \mu_{0}=20, \mu_{1}=30, \theta=\mu_{0}=20, \sigma=10,12.5,20$ and $50, \tau=\sigma$. The first row for each effect size is with type I and type II error rate constraints $(\alpha, \beta)=(0.05,0.2)$ and the second row for each effect size is with $(\alpha, \beta)=(0.05,0.1)$.

\begin{tabular}{|c|c|c|c|c|c|c|c|c|c|c|c|}
\hline \multicolumn{12}{|c|}{ Optimal Design } \\
\hline Effect size & $\sigma$ & $P_{T}$ & $P_{L}$ & Nmax & $n_{1}$ & $\bar{X}_{L}$ & $\bar{X}_{U}$ & PET & $E_{n}$ & Type I Error & Type II Erro1 \\
\hline \multirow[t]{2}{*}{1.0} & 10.0 & 0.946 & 0.100 & 7 & 3 & 21.54 & 35.33 & 0.61 & 4.57 & 0.041 & 0.200 \\
\hline & & 0.940 & 0.088 & 9 & 4 & 20.80 & 32.65 & 0.57 & 6.15 & 0.050 & 0.100 \\
\hline \multirow[t]{2}{*}{0.9} & 11.1 & 0.938 & 0.100 & 8 & 4 & 21.82 & 33.93 & 0.63 & 5.46 & 0.050 & 0.198 \\
\hline & & 0.942 & 0.094 & 11 & 6 & 21.72 & 31.08 & 0.66 & 25.5 & 0.050 & 0.100 \\
\hline \multirow[t]{2}{*}{0.8} & 12.5 & 0.942 & 0.073 & 10 & 5 & 21.11 & 33.90 & 0.59 & 7.08 & 0.049 & 0.200 \\
\hline & & 0.944 & 0.093 & 14 & 7 & 21.30 & 31.54 & 0.62 & 9.69 & 0.050 & 0.100 \\
\hline \multirow[t]{2}{*}{0.7} & 14.3 & 0.949 & 0.100 & 14 & 5 & 20.29 & 36.16 & 0.52 & 9.29 & 0.045 & 0.200 \\
\hline & & 0.948 & 0.100 & 19 & 7 & 20.21 & 33.29 & 0.52 & 12.74 & 0.048 & 0.100 \\
\hline \multirow[t]{2}{*}{0.6} & 16.7 & 0.946 & 0.088 & 18 & 8 & 20.78 & 34.37 & 0.56 & 12.40 & 0.050 & 0.200 \\
\hline & & 0.947 & 0.099 & 25 & 12 & 21.17 & 31.55 & 0.60 & 17.15 & 0.050 & 0.100 \\
\hline \multirow[t]{2}{*}{0.5} & 20.0 & 0.947 & 0.100 & 26 & 12 & 21.20 & 33.86 & 0.59 & 17.74 & 0.050 & 0.199 \\
\hline & & 0.948 & 0.100 & 36 & 18 & 21.31 & 31.20 & 0.62 & 24.87 & 0.050 & 0.099 \\
\hline \multirow[t]{2}{*}{0.4} & 25.0 & 0.949 & 0.100 & 41 & 17 & 20.64 & 36.44 & 0.55 & 27.79 & 0.050 & 0.199 \\
\hline & & 0.950 & 0.100 & 57 & 24 & 20.59 & 32.09 & 0.56 & 38.69 & 0.050 & 0.100 \\
\hline \multirow[t]{2}{*}{0.3} & 33.3 & 0.950 & 0.095 & 72 & 33 & 20.97 & 33.69 & 0.58 & 49.56 & 0.050 & 0.200 \\
\hline & & 0.951 & 0.100 & 101 & 45 & 20.81 & 31.71 & 0.57 & 68.65 & 0.049 & 0.100 \\
\hline \multirow[t]{2}{*}{0.2} & 50.0 & 0.951 & 0.100 & 163 & 72 & 20.89 & 33.82 & 0.57 & 111.20 & 0.050 & 0.200 \\
\hline & & 0.951 & 0.100 & 226 & 103 & 20.87 & 31.54 & 0.58 & 154.66 & 0.050 & 0.100 \\
\hline \multirow[t]{2}{*}{0.1} & 100 & 0.952 & 0.100 & 656 & 273 & 20.59 & 34.13 & 0.55 & 446.01 & 0.050 & 0.200 \\
\hline & & 0.952 & 0.100 & 910 & 398 & 20.71 & 31.72 & 0.57 & 620.32 & 0.050 & 0.100 \\
\hline
\end{tabular}


Table A3.4 Operating characteristics of minimax two-stage design for continuous endpoints with early termination for efficacy. For both designs below, $P_{U}=0.95, \mu_{0}=20, \mu_{1}=30, \theta=\mu_{0}=20, \sigma=10,12.5,20$ and $50, \tau=\sigma$. The first row for each effect size is with type I and type II error rate constraints $(\alpha, \beta)=(0.05,0.2)$ and the second row for each effect size is with $(\alpha, \beta)=(0.05,0.1)$.

\section{Minimax Design}

\begin{tabular}{|c|c|c|c|c|c|c|c|c|c|c|c|}
\hline Effect size & $\sigma$ & $P_{T}$ & $P_{L}$ & Nmax & $n_{1}$ & $\bar{X}_{L}$ & $\bar{X}_{U}$ & PET & $E_{n}$ & Type I Error & Type II Error \\
\hline 1.0 & 10.0 & $\begin{array}{l}0.936 \\
0.942\end{array}$ & $\begin{array}{l}0.100 \\
0.072\end{array}$ & $\begin{array}{l}7 \\
9\end{array}$ & $\begin{array}{l}3 \\
4\end{array}$ & $\begin{array}{l}21.13 \\
20.44\end{array}$ & $\begin{array}{l}34.93 \\
32.72\end{array}$ & $\begin{array}{l}0.58 \\
0.54\end{array}$ & $\begin{array}{l}4.67 \\
6.30\end{array}$ & $\begin{array}{l}0.050 \\
0.048\end{array}$ & $\begin{array}{l}0.176 \\
0.100\end{array}$ \\
\hline 0.9 & 11.1 & $\begin{array}{l}0.938 \\
0.947\end{array}$ & $\begin{array}{l}0.100 \\
0.037\end{array}$ & $\begin{array}{c}8 \\
11\end{array}$ & $\begin{array}{l}4 \\
6\end{array}$ & $\begin{array}{l}21.82 \\
20.40\end{array}$ & $\begin{array}{l}33.93 \\
31.25\end{array}$ & $\begin{array}{l}0.63 \\
0.54\end{array}$ & $\begin{array}{l}5.46 \\
8.29\end{array}$ & $\begin{array}{l}0.050 \\
0.047\end{array}$ & $\begin{array}{l}0.198 \\
0.100\end{array}$ \\
\hline 0.8 & 12.5 & $\begin{array}{l}0.941 \\
0.944\end{array}$ & $\begin{array}{l}0.100 \\
0.093\end{array}$ & $\begin{array}{l}10 \\
14\end{array}$ & $\begin{array}{l}6 \\
7\end{array}$ & $\begin{array}{l}22.61 \\
21.30\end{array}$ & $\begin{array}{l}32.34 \\
31.54\end{array}$ & $\begin{array}{l}0.70 \\
0.62\end{array}$ & $\begin{array}{l}7.19 \\
9.69\end{array}$ & $\begin{array}{l}0.050 \\
0.050\end{array}$ & $\begin{array}{l}0.200 \\
0.100\end{array}$ \\
\hline 0.7 & 14.3 & $\begin{array}{l}0.945 \\
0.945\end{array}$ & $\begin{array}{l}0.059 \\
0.094\end{array}$ & $\begin{array}{l}13 \\
18\end{array}$ & $\begin{array}{c}7 \\
11\end{array}$ & $\begin{array}{l}21.07 \\
22.12\end{array}$ & $\begin{array}{l}33.19 \\
30.21\end{array}$ & $\begin{array}{l}0.59 \\
0.70\end{array}$ & $\begin{array}{c}9.49 \\
13.12\end{array}$ & $\begin{array}{l}0.049 \\
0.050\end{array}$ & $\begin{array}{l}0.200 \\
0.100\end{array}$ \\
\hline 0.6 & 16.7 & $\begin{array}{l}0.945 \\
0.946\end{array}$ & $\begin{array}{l}0.100 \\
0.100\end{array}$ & $\begin{array}{l}18 \\
24\end{array}$ & $\begin{array}{l}10 \\
21\end{array}$ & $\begin{array}{l}22.13 \\
23.96\end{array}$ & $\begin{array}{l}32.62 \\
27.73\end{array}$ & $\begin{array}{l}0.67 \\
0.88\end{array}$ & $\begin{array}{l}12.68 \\
21.36\end{array}$ & $\begin{array}{l}0.050 \\
0.050\end{array}$ & $\begin{array}{l}0.196 \\
0.100\end{array}$ \\
\hline 0.5 & 20.0 & $\begin{array}{l}0.948 \\
0.949\end{array}$ & $\begin{array}{l}0.030 \\
0.052\end{array}$ & $\begin{array}{l}25 \\
35\end{array}$ & $\begin{array}{l}17 \\
20\end{array}$ & $\begin{array}{l}21.55 \\
20.91\end{array}$ & $\begin{array}{l}31.31 \\
30.59\end{array}$ & $\begin{array}{l}0.64 \\
0.59\end{array}$ & $\begin{array}{l}19.92 \\
26.15\end{array}$ & $\begin{array}{l}0.050 \\
0.050\end{array}$ & $\begin{array}{l}0.200 \\
0.100\end{array}$ \\
\hline 0.4 & 25.0 & $\begin{array}{l}0.949 \\
0.948\end{array}$ & $\begin{array}{l}0.034 \\
0.100\end{array}$ & $\begin{array}{l}39 \\
54\end{array}$ & $\begin{array}{l}29 \\
47\end{array}$ & $\begin{array}{l}22.38 \\
23.91\end{array}$ & $\begin{array}{l}30.57 \\
27.76\end{array}$ & $\begin{array}{l}0.71 \\
0.88\end{array}$ & $\begin{array}{l}31.93 \\
47.88\end{array}$ & $\begin{array}{l}0.050 \\
0.050\end{array}$ & $\begin{array}{l}0.200 \\
0.100\end{array}$ \\
\hline 0.3 & 33.3 & $\begin{array}{l}0.949 \\
0.651\end{array}$ & $\begin{array}{l}0.056 \\
0.014\end{array}$ & $\begin{array}{l}69 \\
96\end{array}$ & $\begin{array}{l}61 \\
65\end{array}$ & $\begin{array}{l}24.31 \\
20.51\end{array}$ & $\begin{array}{l}29.01 \\
29.56\end{array}$ & $\begin{array}{l}0.86 \\
0.56\end{array}$ & $\begin{array}{l}62.11 \\
78.65\end{array}$ & $\begin{array}{l}0.050 \\
0.050\end{array}$ & $\begin{array}{l}0.200 \\
0.100\end{array}$ \\
\hline 0.2 & 50.0 & $\begin{array}{l}0.950 \\
0.951\end{array}$ & $\begin{array}{l}0.030 \\
0.003\end{array}$ & $\begin{array}{l}155 \\
215\end{array}$ & $\begin{array}{l}140 \\
172\end{array}$ & $\begin{array}{l}24.16 \\
20.97\end{array}$ & $\begin{array}{l}28.80 \\
25.66\end{array}$ & $\begin{array}{l}0.86 \\
0.61\end{array}$ & $\begin{array}{l}142.16 \\
188.60\end{array}$ & $\begin{array}{l}0.050 \\
0.050\end{array}$ & $\begin{array}{l}0.200 \\
0.100\end{array}$ \\
\hline 0.1 & 100 & $\begin{array}{l}0.950 \\
0.950\end{array}$ & $\begin{array}{l}0.046 \\
0.045\end{array}$ & $\begin{array}{l}619 \\
857\end{array}$ & $\begin{array}{l}596 \\
837\end{array}$ & $\begin{array}{l}25.29 \\
24.73\end{array}$ & $\begin{array}{l}27.92 \\
26.49\end{array}$ & $\begin{array}{l}0.93 \\
0.94\end{array}$ & $\begin{array}{l}597.65 \\
838.11\end{array}$ & $\begin{array}{l}0.050 \\
0.050\end{array}$ & $\begin{array}{l}0.200 \\
0.100\end{array}$ \\
\hline
\end{tabular}


Table A3.5 Operating characteristics of optimal efficacy designs with different priors. (Design parameter: $\mu_{0}=20, \mu_{1}=30, \alpha=$ $0.05, \beta=0.2, \sigma=20$ )

\begin{tabular}{l|cccccccccc}
\hline $\begin{array}{c}\text { Prior } \\
\mathrm{N}\left(\theta, \tau^{2}\right)\end{array}$ & $P_{T}$ & $P_{L}$ & $N_{\max }$ & $n_{1}$ & $\bar{X}_{L}$ & $\bar{X}_{U}$ & PET & $E_{n}$ & $\begin{array}{c}\text { Type I } \\
\text { Error }\end{array}$ & $\begin{array}{c}\text { Type II } \\
\text { Error }\end{array}$ \\
\hline$N\left(20,20^{2}\right)$ & 0.947 & 0.100 & 26 & 12 & 21.20 & 33.86 & 0.59 & 17.74 & 0.050 \\
$N\left(20,8.94^{2}\right)$ & 0.931 & 0.096 & 26 & 11 & 21.15 & 36.07 & 0.58 & 17.31 & 0.050 \\
$N\left(30,20^{2}\right)$ & 0.958 & 0.100 & 26 & 12 & 20.83 & 33.49 & 0.58 & 18.07 & 0.050 \\
$N\left(30,8.94^{2}\right)$ & 0.977 & 0.100 & 26 & 13 & 20.66 & 33.03 & 0.56 & 18.76 & 0.048 \\
\hline
\end{tabular}

Table A3.6 Operating characteristics of minimax efficacy designs with different priors. (Design parameter: $\mu_{0}=20, \mu_{1}=30, \sigma=$ $20, \alpha=0.05, \beta=0.2)$

\begin{tabular}{l|cccccccccc}
\hline $\begin{array}{c}\text { Prior } \\
\mathrm{N}\left(\theta, \tau^{2}\right)\end{array}$ & $P_{T}$ & $P_{L}$ & $N_{\max }$ & $n_{1}$ & $\bar{X}_{L}$ & $\bar{X}_{L}^{\prime}$ & PET & $E_{n}$ & $\begin{array}{c}\text { Type I } \\
\text { Error }\end{array}$ & $\begin{array}{c}\text { Type II } \\
\text { Error }\end{array}$ \\
\hline$N\left(20,20^{2}\right)$ & 0.948 & 0.030 & 25 & 17 & 21.55 & 31.31 & 0.64 & 19.92 & 0.050 & 0.200 \\
$N\left(20,8.94^{2}\right)$ & 0.935 & 0.013 & 25 & 16 & 20.27 & 32.42 & 0.53 & 20.25 & 0.049 \\
$N\left(30,20^{2}\right)$ & 0.957 & 0.068 & 25 & 20 & 23.58 & 29.88 & 0.80 & 20.99 & 0.050 & 0.200 \\
$N\left(30,8.94^{2}\right)$ & 0.975 & 0.100 & 25 & 21 & 24.21 & 29.40 & 0.85 & 21.61 & 0.050 & 0.200 \\
\hline
\end{tabular}




\section{A4 Supplementary simulation results for multi-stage designs.}

Table A4.1 Operating characteristics of Minimax multi-stage design for continuous endpoints. For both designs below, $\mu_{0}=20, \quad \mu_{1}=30, \theta=\mu_{0}=20, \sigma=$ $10,12.5,20$ and $50, \tau=\sigma . P_{L}=0.1$ and $P_{U}=0.95$.

$$
\alpha=0.05, \beta=0.1
$$

\begin{tabular}{cc|ccccc}
\hline Effect & $\sigma$ & $K$ & $P_{T}$ & N.max & Type I Error & Type II Error \\
\hline 1.0 & 10 & 3 & 0.940 & 10 & 0.050 & 0.081 \\
& & 4 & 0.940 & 11 & 0.050 & 0.076 \\
& & & 0.940 & 11 & 0.049 & 0.088 \\
& & 0.940 & 11 & 0.049 & 0.087 \\
& & 7 & 0.942 & 13 & 0.050 & 0.070 \\
& 8 & 0.943 & 15 & 0.048 & 0.051 \\
& 9 & 0.941 & 17 & 0.050 & 0.044 \\
& 10 & 0.969 & 12 & 0.017 & 0.071 \\
\hline & 3 & 0.945 & 16 & 0.049 & 0.078 \\
& 4 & 0.945 & 17 & 0.049 & 0.084 \\
& 5 & 0.960 & 18 & 0.037 & 0.096 \\
& 6 & 0.947 & 18 & 0.049 & 0.084 \\
& 7 & 0.947 & 19 & 0.049 & 0.080 \\
& 8 & 0.950 & 17 & 0.048 & 0.088 \\
& 9 & 0.950 & 20 & 0.048 & 0.093 \\
& 10 & 0.929 & 21 & 0.049 & 0.080 \\
\hline & & & & &
\end{tabular}


Table A4.1 (continued)

$$
\alpha=0.05, \beta=0.1
$$

\begin{tabular}{|c|c|c|c|c|c|c|}
\hline Effect & $\sigma$ & $K$ & $P_{T}$ & N.max & Type I Error & Type II Error \\
\hline \multirow[t]{8}{*}{0.5} & 20 & 3 & 0.950 & 38 & 0.049 & 0.098 \\
\hline & & 4 & 0.952 & 41 & 0.049 & 0.097 \\
\hline & & 5 & 0.953 & 43 & 0.049 & 0.098 \\
\hline & & 6 & 0.954 & 45 & 0.049 & 0.089 \\
\hline & & 7 & 0.955 & 46 & 0.050 & 0.096 \\
\hline & & 8 & 0.956 & 48 & 0.049 & 0.095 \\
\hline & & 9 & 0.957 & 50 & 0.047 & 0.099 \\
\hline & & 10 & 0.951 & 52 & 0.046 & 0.036 \\
\hline \multirow[t]{8}{*}{0.2} & 50 & 3 & 0.953 & 237 & 0.050 & 0.100 \\
\hline & & 4 & 0.955 & 252 & 0.050 & 0.099 \\
\hline & & 5 & 0.957 & 263 & 0.050 & 0.098 \\
\hline & & 6 & 0.960 & 274 & 0.049 & 0.100 \\
\hline & & 7 & 0.961 & 291 & 0.050 & 0.094 \\
\hline & & 8 & 0.970 & 295 & 0.043 & 0.090 \\
\hline & & 9 & 0.963 & 296 & 0.049 & 0.089 \\
\hline & & 10 & 0.965 & 310 & 0.049 & 0.044 \\
\hline
\end{tabular}




\section{A5. Calculation of covariance between $\bar{X}_{n_{i}}$ and $\bar{X}_{n_{j}}$ :}

Let $n_{i}$ denote the cumulative number of patients at $\mathrm{i}^{\text {th }}$ stage and $n_{j}$ denote the cumulative sample size at $\mathrm{j}^{\text {th }}$ stage, where $n_{j}>n_{i}$. Thus, $\bar{X}_{n_{i}}$ denotes the average tumor shrinkage from the $n_{i}$ patients and $\bar{X}_{n_{j}}$ denotes the average tumor shrinkage from the $n_{j}$ patients.

$$
\begin{aligned}
\operatorname{Cov}\left(\bar{X}_{n_{i}}, \bar{X}_{n_{j}}\right)= & \operatorname{Cov}\left(\frac{1}{n_{i}} \sum_{i=1}^{n_{i}} X_{i}, \frac{1}{n_{j}} \sum_{j=1}^{n_{j}} X_{j}\right) \\
= & E\left(\frac{1}{n_{i}} \sum_{i=1}^{n_{i}} X_{i} * \frac{1}{n_{j}} \sum_{j=1}^{n_{j}} X_{j}\right)-E\left(\frac{1}{n_{i}} \sum_{i=1}^{n_{i}} X_{i}\right) E\left(\frac{1}{n_{j}} \sum_{j=1}^{n_{j}} X_{j}\right) \\
& =\frac{1}{n_{i} n_{j}} E\left(\sum_{i=1}^{n_{i}} X_{i} \sum_{j=1}^{n_{j}} X_{j}\right)-\mu^{2} \\
& =\frac{1}{n_{i} n_{j}} E\left(n_{i} \bar{X}_{n_{i}} n_{j} \bar{X}_{n_{j}}\right)-\mu^{2} \\
& =\frac{1}{n_{i} n_{j}} E\left[n_{i} \bar{X}_{n_{i}} *\left(n_{i} \bar{X}_{n_{i}}+\left(n_{j}-n_{i}\right) \bar{X}^{\prime} n_{j}\right)\right]-\mu^{2} \\
& =\frac{1}{n_{i} n_{j}} E\left[n_{i}^{2} \bar{X}_{n_{i}}^{2}+n_{i} \bar{X}_{n_{i}}\left(n_{j}-n_{i}\right) \bar{X}_{n_{j}}^{\prime}\right]-\mu^{2} \\
& =\frac{n_{i}^{2}}{n_{i} n_{j}} E\left[\bar{X}_{n_{i}}^{2}\right]+\frac{n_{i}\left(n_{j}-n_{i}\right)}{n_{i} n_{j}} E\left(\bar{X}_{n_{i}} \bar{X}_{n_{j}}^{\prime}\right)-\mu^{2}
\end{aligned}
$$

Since $E\left[\bar{X}_{n_{i}}^{2}\right]=\operatorname{Var}\left(\bar{X}_{n_{i}}\right)+\left[E\left(\bar{X}_{n_{i}}\right)\right]^{2}, \bar{X}_{n_{i}}$ and $\bar{X}_{n_{j}}^{\prime}$ are independent with both mean $\mu$, where $\bar{X}_{n_{j}}^{\prime}$ is the average tumor shrinkage from the patients $n_{j}-n_{i}$.

$$
\begin{aligned}
\operatorname{Cov}\left(\bar{X}_{n_{i}}, \bar{X}_{n_{j}}\right) & =\frac{n_{i}^{2}}{n_{i} n_{j}}\left(\operatorname{Var}\left(\bar{X}_{n_{i}}\right)+\left[E\left(\bar{X}_{n_{i}}\right)\right]^{2}\right)+\frac{n_{i}\left(n_{j}-n_{i}\right)}{n_{i} n_{j}} E\left(\bar{X}_{n_{i}}\right) E\left(\bar{X}_{n_{j}}^{\prime}\right)-\mu^{2} \\
& =\frac{n_{i}^{2}}{n_{i} n_{j}}\left(\frac{\sigma^{2}}{n_{i}}+\mu^{2}\right)+\frac{n_{i}\left(n_{j}-n_{i}\right)}{n_{i} n_{j}} \mu^{2}-\mu^{2}
\end{aligned}
$$




$$
=\frac{\sigma^{2}}{n_{j}}+\frac{n_{i}^{2}}{n_{i} n_{j}} \mu^{2}+\mu^{2}-\frac{n_{i}^{2}}{n_{i} n_{j}} \mu^{2}-\mu^{2}=\frac{\sigma^{2}}{n_{j}}
$$

Calculation of covariance between $Z_{n_{i}}$ and $Z_{n_{j}}$ from $\bar{X}_{n_{i}}$ and $\bar{X}_{n_{j}}$

$$
\begin{aligned}
& Z_{n_{i}}=\frac{\bar{X}_{n_{i}}-\mu}{\sqrt{\sigma^{2} / n_{i}}} \text { and } Z_{n_{j}}=\frac{\bar{X}_{n_{j}}-\mu}{\sqrt{\sigma^{2} / n_{j}}} \\
& \operatorname{Cov}\left(Z_{n_{i}}, Z_{n_{j}}\right)=\operatorname{Cov}\left(\frac{\bar{X}_{n_{i}}-\mu}{\sqrt{\frac{\sigma^{2}}{n_{i}}}}, \frac{\bar{X}_{n_{j}}-\mu}{\sqrt{\frac{\sigma^{2}}{n_{j}}}}\right)=E\left(\frac{\bar{X}_{n_{i}}-\mu}{\sqrt{\frac{\sigma^{2}}{n_{i}}}} * \frac{\bar{X}_{n_{j}}-\mu}{\sqrt{\frac{\sigma^{2}}{n_{j}}}}\right)-E\left(\frac{\bar{x}_{n_{i}}-\mu}{\sqrt{\frac{\sigma^{2}}{n_{i}}}}\right) E\left(\frac{\bar{X}_{n_{j}}-\mu}{\sqrt{\frac{\sigma^{2}}{n_{j}}}}\right) \\
& =\frac{\sqrt{n_{i} n_{j}}}{\sigma^{2}} E\left[\left(\bar{X}_{n_{i}}-\mu\right)\left(\bar{X}_{n_{j}}-\mu\right)\right]-\frac{\sqrt{n_{i} n_{j}}}{\sigma^{2}} E\left[\bar{X}_{n_{i}}-\mu\right] E\left[\bar{X}_{n_{j}}-\mu\right] \\
& =\frac{\sqrt{n_{i} n_{j}}}{\sigma^{2}} E\left[\bar{X}_{n_{i}} \bar{X}_{n_{j}}-\mu\left(\bar{X}_{n_{i}}+\bar{X}_{n_{j}}\right)+\mu^{2}\right]-0 \\
& =\frac{\sqrt{n_{i} n_{j}}}{\sigma^{2}} E\left[\bar{X}_{n_{i}} \bar{X}_{n_{j}}\right]-\frac{\sqrt{n_{i} n_{j}}}{\sigma^{2}} E\left[\mu\left(\bar{X}_{n_{i}}+\bar{X}_{n_{j}}\right)\right]+\frac{\sqrt{n_{i} n_{j}}}{\sigma^{2}} E\left[\mu^{2}\right] \\
& =\frac{\sqrt{n_{i} n_{j}}}{\sigma^{2}} E\left[\bar{X}_{n_{i}} * \frac{1}{n_{j}} \sum_{j=1}^{n_{j}} X_{j}\right]-\frac{\sqrt{n_{i} n_{j}}}{\sigma^{2}} E\left[\mu\left(\bar{X}_{n_{i}}+\bar{X}_{n_{j}}\right)\right]+\frac{\sqrt{n_{i} n_{j}}}{\sigma^{2}} E\left[\mu^{2}\right] \\
& =\frac{\sqrt{n_{i} n_{j}}}{\sigma^{2}} \frac{1}{n_{j}} E\left[\bar{X}_{n_{i}}\left(n_{i} \bar{X}_{n_{i}}+\left(n_{j}-n_{i}\right) \bar{X}^{\prime}{ }_{n_{j}}\right)\right]-\frac{\sqrt{n_{i} n_{j}}}{\sigma^{2}} E\left[\mu\left(\bar{X}_{n_{i}}+\bar{X}_{n_{j}}\right)\right] \\
& +\frac{\sqrt{n_{i} n_{j}}}{\sigma^{2}} E\left[\mu^{2}\right] \\
& =\frac{1}{\sigma^{2}} \sqrt{\frac{n_{i}}{n_{j}}} E\left[n_{i} \bar{X}_{n_{i}}^{2}+\left(n_{j}-n_{i}\right) \bar{X}_{n_{i}} \bar{X}_{n_{j}}^{\prime}\right]-\frac{\sqrt{n_{i} n_{j}}}{\sigma^{2}} \mu E\left[\bar{X}_{n_{i}}+\bar{X}_{n_{j}}\right]+\frac{\sqrt{n_{i} n_{j}}}{\sigma^{2}} \mu^{2}
\end{aligned}
$$




$$
\begin{aligned}
& =\frac{n_{i}}{\sigma^{2}} \sqrt{\frac{n_{i}}{n_{j}}} E\left[\bar{X}_{n_{i}}^{2}\right]+\frac{1}{\sigma^{2}} \sqrt{\frac{n_{i}}{n_{j}}} E\left[\left(n_{j}-n_{i}\right) \bar{X}_{n_{i}} \bar{X}_{n_{j}}^{\prime}\right]-2 \frac{\sqrt{n_{i} n_{j}}}{\sigma^{2}} \mu^{2}+\frac{\sqrt{n_{i} n_{j}}}{\sigma^{2}} \mu^{2} \\
& =\frac{n_{i}}{\sigma^{2}} \sqrt{\frac{n_{i}}{n_{j}}}\left(\operatorname{Var}\left(\bar{X}_{n_{i}}\right)+\left[E\left(\bar{X}_{n_{i}}\right)\right]^{2}\right)+\frac{1}{\sigma^{2}} \sqrt{\frac{n_{i}}{n_{j}}}\left(n_{j}-n_{i}\right) E\left[\bar{X}_{n_{i}} \bar{X}^{\prime} n_{j}\right] \\
& -\frac{\sqrt{n_{i} n_{j}}}{\sigma^{2}} \mu^{2} \\
& =\frac{n_{i}}{\sigma^{2}} \sqrt{\frac{n_{i}}{n_{j}}}\left(\frac{\sigma^{2}}{n_{i}}+\mu^{2}\right)+\frac{1}{\sigma^{2}} \sqrt{\frac{n_{i}}{n_{j}}}\left(n_{j}-n_{i}\right) E\left[\bar{X}_{n_{i}} \bar{X}^{\prime} n_{j}\right]-\frac{\sqrt{n_{i} n_{j}}}{\sigma^{2}} \mu^{2} \\
& =\sqrt{\frac{n_{i}}{n_{j}}}+\frac{n_{i}}{\sigma^{2}} \sqrt{\frac{n_{i}}{n_{j}}} \mu^{2}+\frac{\sqrt{n_{i} n_{j}}}{\sigma^{2}} n_{j} \mu^{2}-\frac{n_{i}}{\sigma^{2}} \sqrt{\frac{n_{i}}{n_{j}}} \mu^{2}-\frac{\sqrt{n_{i} n_{j}}}{\sigma^{2}} \mu^{2}=\sqrt{\frac{n_{i}}{n_{j}}}
\end{aligned}
$$

\section{Calculation of covariance between $\bar{X}_{n_{i}}$ and $\bar{X}_{n_{j}}$ from $Z_{n_{i}}$ and $Z_{n_{j}}$}

Since $Z_{n_{i}}=\frac{\bar{X}_{n_{i}-\mu}}{\sqrt{\sigma^{2} / n_{i}}}$ and $Z_{n_{j}}=\frac{\bar{X}_{n_{j}}-\mu}{\sqrt{\sigma^{2} / n_{j}}}, \bar{X}_{n_{i}}=Z_{n_{i}} \sqrt{\sigma^{2} / n_{i}}+\mu$ and $\bar{X}_{n_{j}}=Z_{n_{j}} \sqrt{\sigma^{2} / n_{j}}+$ $\mu$

$$
\begin{aligned}
\operatorname{Cov}\left(\bar{X}_{n_{i}}, \bar{X}_{n_{j}}\right) & =\operatorname{Cov}\left(Z_{n_{i}} \sqrt{\sigma^{2} / n_{i}}+\mu, Z_{n_{j}} \sqrt{\sigma^{2} / n_{j}}+\mu\right) \\
& =\operatorname{Cov}\left(Z_{n_{i}} \sqrt{\frac{\sigma^{2}}{n_{i}}}, Z_{n_{j}} \sqrt{\frac{\sigma^{2}}{n_{j}}}\right) \\
& =\frac{\sigma^{2}}{\sqrt{n_{i} n_{j}}} \operatorname{Cov}\left(Z_{n_{i}}, Z_{n_{j}}\right) \\
& =\frac{\sigma^{2}}{\sqrt{n_{i} n_{j}}} \sqrt{\frac{n_{i}}{n_{j}}}=\frac{\sigma^{2}}{n_{j}}
\end{aligned}
$$




\title{
A6. R Package Manual
}

\section{Package 'PPSC'}

\author{
July 17, 2017
}

Type Package

Title PPSC: An R Package for Single-

Arm Phase II Trial Design with Continuous Endpoints based on Predictive Probability

Version 0.1 .0

Author Meng Liu

Emily V. Dressler

Maintainer Meng Liu <mengliu236@gmail.com>

Description This is an $\mathrm{R}$ package to implement the grid search of optimal and minimax designs for continuous endpoints based on predictive probability while meeting both user prespecified type I and type II error rate constaints.

Depends cubature,

R2Cuba,

ggplot2

Imports cubature,

R2Cuba,

ggplot2

License GPL-2

Encoding UTF-8

LazyData true

Suggests knitr

RoxygenNote 6.0 .1 .9000

\section{R topics documented:}

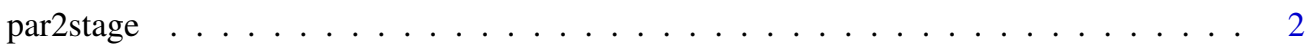

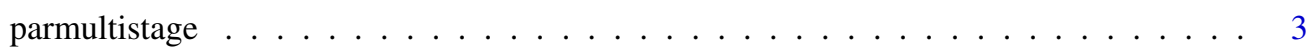

$\begin{array}{lr}\text { Index } & 6\end{array}$ 


\section{Description}

This function allows the user to search for the optimal and minimax predictive probability design for continuous normal endpints in a single-arm two-stage clinical trial setting. "sigma" is the standard deviation for the likelihood function. "tau" is the standard deviation for normal prior distribnution. Both "sigma" and "tau" need input from the investigators and usually should be obtained from the historical control or standard therapy. The ratio of "sigma" to "tau" is related to the effective sample size for the prior.

\section{Usage}

par2stage(sigma, tau, theta, mu0, mu1, pTlow, pThigh, pTby, pLlow, pLhigh, pLby, $\mathrm{pU}$, pre.typeIerror, pre.typeIIerror)

\section{Arguments}

sigma

tau

theta

mu0

mu1

pTlow

pThigh

pTby

pLlow

pLhigh

pLby

$\mathrm{pU}$

pre.typeIerror

pre.typeIIerror

The user pre-specified nominal type II error rate constraint.The default value is 0.2 .

The standard deviation from the user pre-specified normal likelihood function. It should be obtained from previous historical control or standard therapy.

The standard deviation from the user pre-specified normal prior distribution. It should be input from the clinical investigators.

The mean from the user pre-specified normal prior distributions.It should be input from the clinical investigators.

The maximum uninteresting mean from the null hypothesis.

The minimum interesting mean from the alternative hypothesis.

The lower boundary for searching range of pT. pT is usually a large probability above 0.8 . If the searching range for $\mathrm{pT}$ is 0.9 to 0.99 , p Tlow $=0.9$.

The higher boundary for searching range of $\mathrm{pT}$. pT is usually a large probability above 0.8 . If the searching range for $\mathrm{pT}$ is 0.9 to 0.99 , pThigh $=0.99$.

The searching increment for $\mathrm{pT}$. The default value is 0.01 .

The lower boundary for searching range of $\mathrm{pL}$. $\mathrm{pL}$ is usually a small probability below 0.1 . If the searching range for $\mathrm{pL}$ is 0.01 to 0.1 , pLlow $=0.01$.

The higher boundary for searching range of $\mathrm{pL}$. $\mathrm{pL}$ is usually a small probability below 0.1 . If the searching range for $\mathrm{pL}$ is 0.01 to 0.1 , pLhigh $=0.1$.

The searching increment for $\mathrm{pL}$. The default value is 0.01 .

The efficacy boundary for predictive probability. It is a value usually above 0.9 .

The user pre-specified nominal type I error rate constraint. The default value is 0.05 . 


\section{Value}

Returns the operating characteristics of two-stage optimal and minimax designs

Total Sample Size

Total sample size for the two stages

Sample Size for the First Stage

The number of patients required in the first stage

Futility Boundaries at the End of the First Stage

A value under which to stop the trial early for futility at the end of the first stage

Futility Boundaries at the End of the Second Stage

A value under which to claim the trial unsuccessful

Efficacy Boundaries at the End of the First Stage

A value above which to stop the trial early for efficacy at the end of the first stage

Efficacy Boundaries at the End of the Second Stage

A value above which to claim the trial successful

Probability of Early Termination

Probability of early termination under the null hypothesis

Expected Sample Size under HO

Expected sample size under the null hypothesis

Type I Error The Actual type I error rate

Type II Error The Actual type II error rate

pT The actual pT yeilding the desired design

$\mathrm{pL} \quad$ The actual $\mathrm{pL}$ yeilding the desired design

Plot The plot containing stopping boundaries

\section{Examples}

\#Example 1: a two-stage design with mu $=20$ and mu1 $=30$ and stop the trial early only for futility

par2stage $($ sigma $=20$, tau $=20$, theta $=20$, mu $0=20$, mu1 $=30$, pTlow $=0.9$, pThigh $=0.99$, pTby $=0.01$, pLlow $=0.01$, pLhigh $=0.1$, pLby $=0.01, \mathrm{pU}=1$, pre.typeIerror $=0.05$, pre. typeIIerror $=0.2$ )

\#Example 2: a two-stage design with mu $0=20$ and mu1 $=30$ and stop the trial early for both futility and efficacy

par2stage $($ sigma $=20, \operatorname{tau}=20$, theta $=20$, mu $0=20$, mu1 $=30$, pTlow $=0.9$, pThigh $=0.99$, pTby $=0.01$, pLlow $=0.01$, pLhigh $=0.1$, pLby $=0.01, \mathrm{pU}=0.95$, pre.typeIerror $=0.05$, pre.typeIIerror $=0.2$ )

parmultistage

Find the minimax multi-stage design based on predictive probability and plot the stopping boundaries

\section{Description}

This function performs a grid search for the minimax design in a single-arm multi-stage setting. There are many different user-defined options in this function, which allows for different group sizes and choosing continuously monitoring. 


\section{Usage}

parmultistage(continuous, first.look, num.interim, equal.spaced, user.p, sigma, tau, mu0, mu1, theta, pTlow, pThigh, pTby, pL, pU, pre.typeIerror, pre.typeIIerror, plot_yes)

\section{Arguments}

continuous

first.look

num.interim

equal.spaced

user.p

sigma

tau

mu0

mu1

theta

pTlow

pThigh

pTby

$\mathrm{pL}$

$\mathrm{pU}$

pre.typeIerror

The user pre-specified nominal type II error rate constraint.The default value is 0.2 .

plot_yes

The option to choose continuous monitoring or not. The default value is $\mathrm{F}$, representing "no continuous monitoring"

The proportion of the total sample size before the continuously monitoring. The default value is NULL. It should have a value if the value for "continuous" equals to $\mathrm{T}$.

The number of interim looks involved in the trial. The user should specify a value up to 40 if the same sample size at each stage is preferred. That is, when eqaul. spaced $=\mathrm{T}$.

The option to choose if the same sample size at each stage is required. If so, the value is set as T. Otherwise, equal. spaced $=\mathrm{F}$.

When different sample sizes at each stage is desired, the user should specify the cumulative proportion at the end of each stage. For example, if the user want to conduct the interim analysis at 20 for user.p is $c(0.2,0.5,0.8,1)$. If the same sample size at each stage is desired, the value is NULL.

The standard deviation from the user pre-specified normal likelihood function.

The standard deviation from the user pre-specified normal prior distribution.

The maximum uninteresting mean from the null hypothesis.

The minimum interesting mean from the alternative hypothesis.

The mean from the user pre-specified normal prior distribution.

The lower boundary for searching range of pT. pT is usually a large probability above 0.8. If the searching range for pT is 0.9 to 0.99 , pTlow $=0.9$.

The higher boundary for searching range of $\mathrm{pT}$. pT is usually a large probability above 0.8. If the searching range for $\mathrm{pT}$ is 0.9 to 0.99 , pThigh $=0.99$.

The searching increment for $\mathrm{pT}$. The default value is 0.01 .

The futility boundary for predictive probability. It is a value usually below 0.1 .

The efficacy boundary for predictive probability. It is a value usually above 0.9 .

The user pre-specified nominal type I error rate constraint. The default value is 0.05 .

The option to choose if displaying the stopping boundary plot.

\section{Details}

parmultistage utilized the grid search strategy to implement the search in a user pre-specified range for $\mathrm{pT}$ and identify the minimax design. 


\section{Value}

Returns the operating characteristics of the minimax design

Cumulative sample size for each interim look

A vector containing the cumulative sample size at the end of each stage for interim analysis

Futility Boundaries

A vector containing the futility stopping boundaries for each interim look

Efficacy Boundaries

A vector containing the efficacy stopping boundaries for each interim look

Probability of Early Termination

Probability of early termination under the null hypothesis

Expected Sample Size under HO

Expected sample size under the null hypothesis

Type I Error The Actual type I error rate

Type II Error The Actual type II error rate

pT

The actual pT yeilding the desired design

Plot

The plot containing stopping boundaries

\section{Examples}

\#Example 1 : Equal sample size at each interim look

parmultistage (continuous $=\mathrm{F}$, first.look $=$ NULL, num.interim $=2$, equal. spaced $=\mathrm{T}$, user. $\mathrm{p}=\mathrm{NULL}$, sigma $=20$, tau $=20$, mu $0=20$, mu1 $=30$, theta $=20, \mathrm{pTlow}=0.9$,

pThigh $=0.99$, pTby $=0.01, \mathrm{pL}=0.1, \mathrm{pU}=0.95$, pre.typeIerror $=0.05$,

pre.typeIIerror $=0.2$, plot_yes $=\mathrm{T}$ )

\#Example 2: Unequal sample size for at each interim look

parmultistage (continuous $=\mathrm{F}$, first.look $=\mathrm{NULL}$, num. interim $=3$, equal. spaced $=\mathrm{F}$, user. $p=c(0.1,0.4,0.8,1.0)$, sigma $=20$, tau $=20$, mu $0=20$, mu1 $=30$, theta $=20$, pTlow $=0.9$, pThigh $=0.99$, pTby $=0.01, \mathrm{pL}=0.1, \mathrm{pU}=0.95$, pre.typeIerror $=0.05$, pre.typeIIerror $=0.2$, plot_yes $=\mathrm{T}$ )

\#Exmaple 3: Continuouly monitor after $50 \%$ of sample size are enrolled parmultistage (continuous $=\mathrm{T}$, first.look $=0.5$, num. interim $=$ NULL, equal. spaced $=\mathrm{F}$, user $\cdot \mathrm{p}=\mathrm{NULL}$, sigma $=20$, tau $=20$, mu0 $=20$, mu1 $=30$, theta $=20$, pTlow $=0.9$, pThigh $=0.99$, pTby $=0.01, \mathrm{pL}=0.1, \mathrm{pU}=0.95$, pre.typeIerror $=0.05$, pre.typeIIerror $=0.2$, plot_yes $=\mathrm{T}$ ) 


\section{Index}

\section{*Topic PPSC}

par2stage, 2

parmultistage, 3

par2stage, 2

parmultistage, 3 


\section{BIBLIOGRAPHY}

1. Eisenhauer, E.A., et al., New response evaluation criteria in solid tumours: revised RECIST guideline (version 1.1). Eur J Cancer, 2009. 45(2): p. 228-47.

2. Adjei, A.A., M. Christian, and P. Ivy, Novel designs and end points for phase II clinical trials. Clin Cancer Res, 2009. 15(6): p. 1866-72.

3. Seymour, L., et al., The design of phase II clinical trials testing cancer therapeutics: consensus recommendations from the clinical trial design task force of the national cancer institute investigational drug steering committee. Clin Cancer Res, 2010. 16(6): p. 1764-9.

4. Booth, C.M., et al., Design and conduct of phase II studies of targeted anticancer therapy: recommendations from the task force on methodology for the development of innovative cancer therapies (MDICT). Eur J Cancer, 2008. 44(1): p. 25-9.

5. Wason, J.M., A.P. Mander, and T.G. Eisen, Reducing sample sizes in two-stage phase II cancer trials by using continuous tumour shrinkage end-points. Eur J Cancer, 2011. 47(7): p. 983-9.

6. Altman, D.G. and P. Royston, The cost of dichotomising continuous variables. BMJ, 2006. 332(7549): p. 1080.

7. Claret, L., et al., Evaluation of tumor-size response metrics to predict overall survival in Western and Chinese patients with first-line metastatic colorectal cancer. J Clin Oncol, 2013. 31(17): p. 2110-4.

8. Delahunt, B., et al., Prognostic importance of tumor size for localized conventional (clear cell) renal cell carcinoma: assessment of TNM T1 and T2 tumor categories and comparison with other prognostic parameters. Cancer, 2002. 94(3): p. 658-64.

9. Simon, R., R.E. Wittes, and S.S. Ellenberg, Randomized phase II clinical trials. Cancer Treat Rep, 1985. 69(12): p. 1375-81.

10. Rubinstein, L.V., et al., Design issues of randomized phase II trials and a proposal for phase II screening trials. J Clin Oncol, 2005. 23(28): p. 7199-206.

11. Rosner, G.L., W. Stadler, and M.J. Ratain, Randomized discontinuation design: application to cytostatic antineoplastic agents. J Clin Oncol, 2002. 20(22): p. 447884.

12. Cannistra, S.A., Phase II trials in journal of clinical oncology. J Clin Oncol, 2009. 27(19): p. 3073-6.

13. Kamat, A.M., et al., Definitions, End Points, and Clinical Trial Designs for NonMuscle-Invasive Bladder Cancer: Recommendations From the International Bladder Cancer Group. J Clin Oncol, 2016. 34(16): p. 1935-44.

14. Whitehead, J., Stopping clinical trials by design. Nat Rev Drug Discov, 2004. 3(11): p. 973-7.

15. Emerson, S.S. and T.R. Fleming, Interim analyses in clinical trials. Oncology (Williston Park), 1990. 4(3): p. 126-33; discussion 134, 136.

16. Fossa, S.D. and E. Skovlund, Interim analyses in clinical trials: why do we plan them? J Clin Oncol, 2000. 18(24): p. 4007-8.

17. Whitehead, A., et al., Incorporating intermediate binary responses into interim analyses of clinical trials: a comparison of four methods. Stat Med, 2008. 27(10): p. 1646-66. 
18. Gehan, E.A., The determinatio of the number of patients required in a preliminary and a follow-up trial of a new chemotherapeutic agent. J Chronic Dis, 1961. 13: p. 346-53.

19. Simon, R., Optimal two-stage designs for phase II clinical trials. Control Clin Trials, 1989. 10(1): p. 1-10.

20. Chen, T.T., Optimal three-stage designs for phase II cancer clinical trials. Stat Med, 1997. 16(23): p. 2701-11.

21. Bryant, J. and R. Day, Incorporating toxicity considerations into the design of twostage phase II clinical trials. Biometrics, 1995. 51(4): p. 1372-83.

22. Conaway, M.R. and G.R. Petroni, Bivariate sequential designs for phase II trials. Biometrics, 1995. 51(2): p. 656-64.

23. Jin, H., Alternative designs of phase II trials considering response and toxicity. Contemp Clin Trials, 2007. 28(4): p. 525-31.

24. Lin, X., R. Allred, and G. Andrews, A two-stage phase II trial design utilizing both primary and secondary endpoints. Pharm Stat, 2008. 7(2): p. 88-92.

25. Jung, S.H., M. Carey, and K.M. Kim, Graphical search for two-stage designs for phase II clinical trials. Control Clin Trials, 2001. 22(4): p. 367-72.

26. Berry, D.A., Bayesian clinical trials. Nat Rev Drug Discov, 2006. 5(1): p. 27-36.

27. Spiegelhalter, D., et al., Bayesian methods in health technology assessment: a review. Health Technology Assessment, 2000. 4(38): p. 130.

28. Thall, P.F. and R. Simon, Practical Bayesian guidelines for phase IIB clinical trials. Biometrics, 1994. 50(2): p. 337-49.

29. Tan, S.B. and D. Machin, Bayesian two-stage designs for phase II clinical trials. Stat Med, 2002. 21(14): p. 1991-2012.

30. Sambucini, V., A Bayesian predictive two-stage design for phase II clinical trials. Stat Med, 2008. 27(8): p. 1199-224.

31. Sambucini, V., A Bayesian predictive strategy for an adaptive two-stage design in phase II clinical trials. Stat Med, 2010. 29(13): p. 1430-42.

32. Brutti, P., et al., Continuous endpoints in Bayesian two-stage designs. J Biopharm Stat, 2016. 26(5): p. 966-77.

33. Mayo, M.S. and B.J. Gajewski, Bayesian sample size calculations in phase II clinical trials using informative conjugate priors. Control Clin Trials, 2004. 25(2): p. 157-67.

34. Wang, Y.G., et al., Bayesian designs with frequentist and Bayesian error rate considerations. Stat Methods Med Res, 2005. 14(5): p. 445-56.

35. Shi, H. and G. Yin, Bayesian two-stage design for phase II clinical trials with switching hypothesis. Bayesian Analysis, 2017. 12(1): p. 31-51.

36. Herson, J., Predictive probability early termination plans for phase II clinical trials. Biometrics, 1979. 35(4): p. 775-83.

37. Lee, J.J. and D.D. Liu, A predictive probability design for phase II cancer clinical trials. Clin Trials, 2008. 5(2): p. 93-106.

38. Yin, G., N. Chen, and J.J. Lee, Phase II trial design with Bayesian adaptive randomization and predictive probability. J R Stat Soc Ser C Appl Stat, 2012. 61(2).

39. Dong, G., et al., A Bayesian-frequentist two-stage single-arm phase II clinical trial design. Stat Med, 2012. 31(19): p. 2055-67. 
40. Cox, D.R., Principles of statistical inference. Cambridge University Press, Cambridge, New York, 2006.

41. Young, G.A. and L.R. Smith, Essentials of statistical inference. Cambridge series in statisticial and probilistic mathematics, 2010.

42. Bolstad, M.W., Introduction to Bayesian statistics. John Wiley, Hoboken, N.J. 2nd edition, 2007.

43. Kruschke, K.J., Doing Bayesian data analysis: a tutorial with $R$ and BUGS. . Academic Press, Burlington, MA, 2011.

44. Sanogo, M., E. Abatih, and C. Saegerman, Bayesian versus frequentist methods for estimating true prevalence of disease and diagnostic test performance. Vet J, 2014. 202(2): p. 204-7.

45. Berry, S.M., et al., Bayesian adaptive methods for clinical trials. Chapman \& Hall/ CRC Biostatistics Series, 2010.

46. Spiegelhalter, D., K. Abrams, and P.M. Jonathan, Bayesian approaches to clinical trials and health-care evaluation. 2004.

47. Efron, B., Bayesians, Frequentists, and Scientists. Journal of American Statisticial Association, 2005. 100: p. 1-5.

48. Bacchieri, A. and G. Della Cioppa, Fundamentals of clinical research bridging medicine, statistics, and operations. 2007.

49. Grossman, J., et al., A unified method for monitoring and analysing controlled trials. Stat Med, 1994. 13(18): p. 1815-26.

50. Freedman, L.S., D.J. Spiegelhalter, and M.K. Parmar, The what, why and how of Bayesian clinical trials monitoring. Stat Med, 1994. 13(13-14): p. 1371-83; discussion 1385-9.

51. Coffey, C.S. and J.A. Kairalla, Adaptive clinical trials: progress and challenges. Drugs R D, 2008. 9(4): p. 229-42.

52. Kairalla, J.A., et al., Adaptive trial designs: a review of barriers and opportunities. Trials, 2012. 13: p. 145.

53. Lavin, P.T., An alternative model for the evaluation of antitumor activity. Cancer Clin Trials, 1981. 4(4): p. 451-7.

54. Karrison, T.G., et al., Design of phase II cancer trials using a continuous endpoint of change in tumor size: application to a study of sorafenib and erlotinib in non small-cell lung cancer. J Natl Cancer Inst, 2007. 99(19): p. 1455-61.

55. Wason, J.M., A.P. Mander, and S.G. Thompson, Optimal multistage designs for randomised clinical trials with continuous outcomes. Stat Med, 2012. 31(4): p. 30112.

56. Chin-Fu, H., C. Shein-Chung, and L. Jen-pei, A Two-Stage Design for Drug Screening Trials Based on Continuous Endpoints. Drug Information Journal, 2008. 42(3): p. 253-262.

57. Whitehead, J., E. Valdes-Marquez, and A. Lissmats, A simple two-stage design for quantitative responses with application to a study in diabetic neuropathic pain. Pharm Stat, 2009. 8(2): p. 125-35.

58. Wason, J.M. and A.P. Mander, Minimizing the maximum expected sample size in two-stage Phase II clinical trials with continuous outcomes. J Biopharm Stat, 2012. 22(4): p. 836-52. 
59. P.Brutti, F.D. Santis, and S.Gubbiotti, Robust Bayesian monitoring of sequential trials. METRON, 2013. 71(1): p. 81-95.

60. Dmitrienko, A. and M.D. Wang, Bayesian predictive approach to interim monitoring in clinical trials. Stat Med, 2006. 25(13): p. 2178-95.

61. Fleming, T.R., One-sample multiple testing procedure for phase II clinical trials. Biometrics, 1982. 38(1): p. 143-51.

62. Pocock, S.J., Group sequential methods in the design and analysis of clincial trials. . Biometrika, 1977. 64: p. 191-199.

63. O'Brien, P.C. and T.R. Fleming, A multiple testing procedure for clinical trials. Biometrics, 1979. 35(3): p. 549-56.

64. Bruno, R. and L. Claret, On the use of change in tumor size to predict survival in clinical oncology studies: toward a new paradigm to design and evaluate phase II studies. Clin Pharmacol Ther, 2009. 86(2): p. 136-8.

65. Clarke, J.M., X. Wang, and N.E. Ready, Surrogate clinical endpoints to predict overall survival in non-small cell lung cancer trials-are we in a new era? Transl Lung Cancer Res, 2015. 4(6): p. 804-8.

66. Henry, N.L. and D.F. Hayes, Cancer biomarkers. Mol Oncol, 2012. 6(2): p. 140-6.

67. Morita, S., P.F. Thall, and P. Muller, Determining the effective sample size of a parametric prior. Biometrics, 2008. 64(2): p. 595-602.

68. Cohen, J., Statistical power analysis for the behavioral sciences. 2nd ed. 1988, Hillsdale, N.J.: L. Erlbaum Associates. xxi, 567 p.

69. (FDA), F.a.D.A., Guidance for the sue of Bayesian statistics in medical device clinical trials, 2010. . 2010.

70. Ensign, L.G., et al., An optimal three-stage design for phase II clinical trials. Stat Med, 1994. 13(17): p. 1727-36.

71. Wolchok, J.D., et al., Guidelines for the evaluation of immune therapy activity in solid tumors: immune-related response criteria. Clin Cancer Res, 2009. 15(23): p. 7412-20.

72. Ribas, A., B. Chmielowski, and J.A. Glaspy, Do we need a different set of response assessment criteria for tumor immunotherapy? Clin Cancer Res, 2009. 15(23): p. 7116-8.

73. Liu, M. and V.E. Dressler, A Predictive Probability Two-Stage Interim Design for Phase II Clinical Trials with Continuous Endpoints. Submitted for publication.

74. Jennison, C.T., B.W., Group sequential methods with applications to clinical trials. 2000.

75. Genz, A. and F. Bretz, Methods ofr the computation of multivariate t-probabilities Journal of Computational and Graphical Statistics, 2002. 11: p. 950-971.

76. Johnson, S.G. and B. Narasimhan, cubature: Adaptive multivariate integration over hypercubes. 2013.

77. Hahn, T., A. Bouvier, and K. Kieu, R2Cuba: Multidimension Numerical Integration. 2015.

78. Chen, K. and M. Shan, Optimal and minimax three-stage designs for phase II oncology clinical trials. Contemp Clin Trials, 2008. 29(1): p. 32-41.

79. Wickham, H., ggplot2: Elegant Graphics for Data Analysis. 2009.

80. Chang, M.N., et al., Designs for group sequential phase II clinical trials. Biometrics, 1987. 43(4): p. 865-74. 
81. Lunn, D.J., et al., WinBUGS - a Bayesian modelling framework: concepts, structure, and extensibility. . Statistics and Computing, 2000. 10: p. 325-337.

82. Sturtz, S., U. Ligges, and A. Gelman, R2WinBUGS: A Package for Running WinBUGS from R. Journal of Statistical Software, 2005. 12(3): p. 1-16.

83. Sit, T., et al., Design and analysis of clinical trials in the presence of delayed treatment effect. Stat Med, 2016. 35(11): p. 1774-9.

84. $\mathrm{Xu}, \mathrm{Z}$., et al., Designing therapeutic cancer vaccine trials with delayed treatment effect. Stat Med, 2017. 36(4): p. 592-605.

85. He, P.S., Z, A novel design for randomized immuno-oncology clinical trials with potentially delayed treatment effects. Contemp Clin Trials, 2015. 1(30): p. 28-31. 


\section{VITA}

\section{EDUCATION}

M.S. Cellular and Molecular Biology

B.S. Biotechnology and Biology Science

\section{PROFESSIONAL EXPERIENCE}

Graduate Research Assistant

Applied Statistics Lab, University of Kentucky

2012-2013

Graduate Teaching Assistant

College of Public Health, University of Kentucky

Graduate Research Assistant

Markey Cancer Center, University of Kentucky

2014-2017

Summer Intern Genentech

Graduate Research Assistant

University of Nevada, Reno

2009-2012

\section{PROFESSIONAL AWARD AND HONORS}

MBSW Student Travel Grant

Academic Enhancement Scholarship, University of Kentucky

Floyd Wright Scholarship, University of Kentucky

2015

\section{PUBLICATION}

1. Dolecek T.A., Dressler E.V., Thakkar J.P., Liu M., Al-Qaisi A, Villano J.L. Descriptive epidemiology of Meningiomas post pubic law 107-206-the benign brain tumor cancer registries act, Cancer 2015, 121(14):2400-10.

2. Knight J.R, Bush H.M, Mase W.A., Riddell M.C., Liu M., Holsinger J.W. The impact of emotional intelligence on conditions of trust among leaders at the Kentucky Department for Public Health, frontiers in Public Health 2015, 3 (2296-2565)

3. Schoenberg N.E., Studts C.R., Shelton B.J., Liu M., Clayton R., Bispo J.B., Fieldds N., Dignan M., Cooper T. A randomized controlled trial of a faith-placed, lay health 
advisor delivered smoking cessation intervention for rural residents. Prev Med Rep. 2016 Jun; 3: $317-323$

4. $\quad$ Butts A.R., Bachmeier C.C., Dressler E.V., Liu M., Cowden A., Talbert J., Adams V.R. Association of time to antibiotics and clinical outcomes in adult hematologic malignancy patients with febrile neutropenia. J Oncol Pharm Pract. 2017 Jun; 23(4): 287283

5. $\quad$ Lefringhouse J.R., Elder J.W., Baldwin L.A., Miller R.W., DeSimone C.P., Van Naqell JR, Samoyoa L.M., West D.S., Dressler V., Liu M., Ueland F. R. Prospective validation of an intraoperative algorithm to determine the extent of surgical staging in early endometrial cancer. Gynecol Oncol. 2017 Apr; 145(1): 50-54

6. Dressler E.V., Dolecek T.A., Liu M., Villano J.L. Demographics, patterns of care, and survival in pediatric medulloblastoma. J Neurooncol. 2017 May; 132(3): 497-506

7. Alfonso K.P., Kaufman M.R., Dressler E.V., Liu M., Aouad R.K. Otolaryngology consultation tracheostomies and complex patient population. Am J Otolaryngol. 2017 Jun; 8. Pan Z.M., Zhou Y., Liu B., You M., Liu M., Jiao X.A. Enhancement of the humoral immune response against the attenuated Salmonella typhimurium harboring Newcastle disease virus DNA vaccine by C3d molecule, Journal of Agricultural Biotechnology. 2009, 17(5):745-749

9. Cai W.T., Liu M., Pan Z.M. The development of Toll or Toll-free vaccine adjuvant. Progress in Veterinary Medicine. 2007, 28(11): 54-58. 